\title{
6 Strukturparallelen zwischen Konstruktionen und Frames
}

\author{
[I]nsights into the mechanics of the grammar as \\ a whole can be brought out most clearly by the \\ work of factoring out the constituent elements \\ of the most complex constructions.
}

(Fillmore 1988: 36)

Konstruktionen und Frames zeichnen sich durch eine wesentliche Gemeinsamkeit aus: Sie besitzen innere Strukturen, die sich mehr oder weniger direkt miteinander vergleichen lassen (vgl. Laviola et al. 2017: 194; Ziem 2020a: 26). ${ }^{1}$ Für die semantische Beschreibung einer Konstruktion nur global auf einen Frame $\mathrm{zu}$ verweisen, wie es besonders in konstruktikographischer Perspektive bisweilen geschieht, reicht deshalb nicht aus. ${ }^{2}$ Dass eine globale Zuweisung eines Konstruktions-Frames zu der Formseite einer Konstruktion nicht die einzige zeichenhafte Relation ist, die es zu beschreiben gilt, betont bereits Langacker (1987: 91): „In a grammatical construction, the global correspondence between semantic and phonological structures is resolvable into a number of more specific correspondences.“ Möchte man etwa erklären, wie sich die semantischen Eigenschaften der Strukturelemente einer Konstruktion und denen ihrer Konstrukte zusammensetzen, bietet es sich an, gerade diese Korrespondenzen in den Blick zu nehmen, diese Strukturelemente also mit FE der Frames, die als lexikalische Frames und Konstruktions-Frames infrage kommen, zu vergleichen. Dieser Vergleich zwischen den Strukturelementen von Konstruktionen und Frames ist zugleich Voraussetzung, um zentrale Eigenschaften einer Konstruktion zu erfassen, insbesondere ihr Koerzionspotenzial (Abschnitte 5.5 und 7.4) und ihre Produktivität (Abschnitte 5.6 und 7.5), die als semantische Parameter für Konstruktionen in die konstruktikographische Beschreibung einer Konstruktion Eingang finden müssen.

Es überrascht kaum, dass bei der überschaubaren Anzahl von Studien, die Frames für die semantische Beschreibung von Konstruktionen heranziehen, nur die wenigsten Analysen auf die Strukturparallelen von Konstruktionen und Fra-

1 Schmid (2020: 69) geht sogar noch weiter und weist darauf hin, dass Frames ebenso wie syntagmatische Muster (also: Konstruktionen) über Slot-Filler-Strukturen verfügen, also Leerstellen aufweisen, die durch Füllwerte besetzt werden können.

2 Schon Geeraerts (1998: 185, 188) sieht es als selbstverständlich an, dass die semantische Beschreibung einer Konstruktion nur in Bezug auf ihre syntaktische Struktur geschehen kann. 
mes eingehen. Die konstruktikographische Literatur ist eine der wenigen Ausnahmen. Als bisher kanonische Gegenüberstellung der Analysekategorien von FrameNet mit denjenigen der Konstruktikographie kann die von Lee-Goldman \& Petruck (2018: 36) aufgestellte, in Tabelle 6.1 ins Deutsche übertragene Zuordnung gelten. ${ }^{3}$

Tab. 6.1: Parallelen zwischen den Strukturelementen von FrameNet-Frames und Konstruktionen (nach Lee-Goldman \& Petruck 2018: 36)

\begin{tabular}{ll}
\hline FrameNet & Konstruktikon \\
\hline Frame & Konstruktion \\
Lexikalische Einheit (LE) bzw. frame-evozieren- & Konstruktionsevozierendes Element (KEE) \\
des Element (FEE) & \\
Frame-Element (FE) & Konstruktionselement (KE) \\
Lexikographische Annotation & Konstruktikographische Annotation \\
\hline
\end{tabular}

Wie ich zu Beginn dieses Kapitels zeigen möchte, ist diese Gegenüberstellung jedoch nicht unproblematisch. Noch am unstrittigsten sind die beiden untersten Zeilen von Tabelle 6.1: Lexikographische und konstruktikographische Annotation unterscheiden sich lediglich in ihrem zu beschreibenen Phänomenbereich - LE oder (syntaktische) Konstruktionen -, wenngleich sie mit unterschiedlichen Kategorien arbeiten, die in der Annotation unterschieden werden müssen. ${ }^{4}$ Diesen Kategorien, zu denen FE und KE gehören, kommt grundsätzlich derselbe strukturierende Status zu: Beide sind Strukturelemente frame-semantisch bzw. konstruktikographisch zu erfassender Entitäten, in einem Falle Frames, im anderen Konstruktionen. In dieser strukturierenden Parallele erschöpfen sich ihre Gemeinsamkeiten aber schnell. Ob FE und KE tatsächlich immer direkt und eineindeutig aufeinander bezogen werden können, um eine Konstruktion semantisch zu beschreiben, ist alles andere als empirisch gesichert und stellt obendrein a priori eine Generalisierung dar, die, wie ich in diesem Kapitel zeigen möchte, auf TypeEbene - also auf Ebene der Konstruktion und damit auf Ebene der KE - nicht möglich ist. Ein noch gravierenderes Problem liegt bei der angenommenen Parallelität

3 Die Blaupause dafür ist die initiale Gegenüberstellung von FrameNet- und konstruktikographischer Annotation bei Fillmore (2008a: 58-59), allerdings sind die Parallelen zwischen den Strukturelementen von Konstruktionen und Frames dort nur implizit angesprochen.

4 Wie bereits erwähnt kennzeichne ich diesen Unterschied dadurch, dass frame-semantische Annotationen lexikalischer Frames durch die Annotation des Frame-Namens auf die Target-LE und deren Tiefstellung gekennzeichnet sind, während in konstruktikographischen Annotationen lediglich KE, KEE und KorE annotiert sind, wobei das Konstrukt innerhalb des Belegs durch geschweifte Klammern gekennzeichnet ist. 
zwischen LE und KEE sowie derjenigen globalen zwischen Frames und Konstruktionen vor. ${ }^{5}$ Dazu schreiben Lee-Goldman \& Petruck (2018):

The FEE is an indication of the specific semantic frame's relevance to interpretation, just as a CEE indicates that a construction may license the current linguistic structure. Likewise, both frames and constructions have their own distinctive elements (FEs and CEs, respectively), with annotation from natural language corpora illustrating these elements. (Lee-Goldman \& Petruck 2018: 36$)^{6}$

Bei genauerem Hinsehen wird deutlich, dass diese Gegenüberstellung - wie ich bereits in Unterabschnitt 2.3.1 erwähnt habe - auf einer rein terminologischen Analogie beruht. Sie betrachtet lediglich Frames und Konstruktionen als Ganzes und getrennt voneinander und nicht in Bezug auf ihre konstruktionssemantische und konstruktikographische Verbindung. Dieses Problem betrifft vor allem die ersten beiden Zeilen der Tabelle. Während die Gegenüberstellung von Frames und Konstruktionen vor dem Hintergrund ihres jeweiligen Status als zu beschreibende Zieleinheiten (Targets) plausibel ist, wird deutlich, dass die Parallele zwischen LE bzw. FEE7 und KEE rein terminologisch ist und sich nur an ihrer Funktion als ,evozierend' orientiert ${ }^{8}$ bzw. daran, dass „[a] CEE thus constitutes the lexical 'anchor' of a construction.“ (Ziem \& Flick 2018: Abschn. 3.1). Dass die Funktion des ,Evozierens' beider Elemente jedoch in Bezug auf das jeweilige Target eine andere ist, wird nicht deutlich gemacht. Klarer ausgedrückt: LE evozieren Frames, KEE evozieren Konstruktionen. ${ }^{9}$ Was zunächst trivial klingt, wird umso wichtiger, wenn

5 KorE sind in der Gegenüberstellung von Lee-Goldman \& Petruck (2018: 36) nicht enthalten, da sie, wie in Unterabschnitt 2.3.1 erwähnt, als Analysekategorie für das Konstruktikon des Deutschen entwickelt wurden, weshalb sie in anderen Konstruktikon-Projekten keine Berücksichtigung finden.

6 Wenngleich Fillmore (2008a: 58) nicht den Terminus des KEE verwendet, mag die Gegenüberstellung bei ihm eine ähnliche sein, wenn es für Frames heißt, dass „,[a]nnotations identify the LU, the FEs, and the GFs [grammatische Funktionen, A.W.] and PTs [Phrasentypen, A.W.] of the segments marked off“ und er parallel für Konstruktionen schreibt: „Annotations contain labels for the CEs and identify, for lexically marked constructions, the relevant lexical material.“

7 Obwohl Ruppenhofer et al. (2016: 11) die Begriffe der LE und des FEE als synonym betrachten, sind sie dies keineswegs: Sie beziehen sich zwar auf dieselbe Entität, sagen aber Unterschiedliches darüber aus (Alexander Ziem, persönliche Kommunikation). Bei Fillmore, Lee-Goldman \& Rhomieux (2012: 322) findet sich gar der Hinweis, dass Konstrukte FEE sein können. Um Missverständnisse zu vermeiden, werde ich fortan nur noch von LE sprechen.

8 Ganz davon abgesehen, dass theoretisch überhaupt nicht geklärt ist, was es heißt, eine Konstruktion - im Gegensatz zu einem Frame - zu ,evozieren'. Vgl. dazu Abschnitt 8.1.

9 Vgl. dazu auch die Definition bei Ziem \& Flick (2018: Abschn. 3.1): ,Just like a frame-evoking element in FrameNet, a CEE provides an explicit link to the respective target structure (here: the construction).“ 
man die Relationen umdreht: LE evozieren keine Konstruktionen und KEE evozieren nicht zwangsläufig Frames. ${ }^{10}$ Deshalb muss die Gegenüberstellung in Tabelle 6.1 korrigiert werden, was im Übrigen auch die erste Zeile betrifft. Das konstruktikographische Pendant zu LE sind Konstruktionen, nicht KEE (vgl. auch Bäckström, Lyngfelt \& Sköldberg 2014: 29). ${ }^{11}$ Im Anschluss an das obige Zitat bemerken LeeGoldman \& Petruck (2018: 36) dies sogar selbst: „At the same time, a construction may be compared to a lexical unit, as both are connected in analogous ways to semantic frames.“

Diese subtilen, aber ernstzunehmenden Probleme möchte ich zum Anlass nehmen, in diesem Kapitel systematische Analysen zu den Strukturparallelen zwischen Konstruktionen und Frames durchzuführen. Ich nehme dafür die ersten drei Zeilen von Tabelle 6.1 in den Blick. Zunächst nehme ich in Abschnitt 6.1 einige grundsätzliche Klärungen zum Verhältnis zwischen Konstruktionen und Frames und der semantischen Motivierung von Konstruktionen und ihren Konstrukten vor. Im Zuge dessen argumentiere ich dafür, nicht KE und FE - also Einheiten auf Type-Ebene - gegenüberzustellen, sondern auf die Token-Ebene $\mathrm{zu}$ wechseln und die semantische Motivierung von KtE durch FE zu untersuchen. Diese Notwendigkeit des Wechsels auf die Token-Ebene deutet auch Ziem (2020a) an:

Da [...] die frame-semantischen Annotationskategorien und solche zur Analyse von grammatischen Konstruktionen strukturell übereinstimmen [...], ist es auch möglich, Frames mit Konstruktionen sowie (Instanzen von) Frame-Elementen mit (Instanzen von) Konstruktionselementen zu verbinden. Dies ist nötig, um mittels Frames ,reiche‘ Bedeutungen von Konstruktionen - also Bedeutungen, die über die durch die KE grammatisch kodierten Bedeutungsaspekte hinausgehen - sowie semantische Beschränkungen eines KE zu erfassen. (Ziem 2020a: 26)

In Abschnitt 6.2 erörtere ich dann ebendiese Parallelen zwischen KtE und FE für die reflexive Bewegungskonstruktion, um im Anschluss daran in Abschnitt 6.3 analog die Parallelen zwischen KEE und FE, ebenfalls für die reflexive Bewegungskonstruktion, zu untersuchen. Die Betrachtung der Konstitution von KonstruktFrames (Abschnitt 4.4) spielt für diese Zwecke eine zentrale Rolle. In Abschnitt 6.4 stehen schließlich einige Sonderfälle im Vordergrund, die aus der zuvor ent-

$10 \mathrm{Zu}$ letzterem Aspekt vgl. Unterabschnitt 8.3.1.

11 Bei Boas, Dux \& Ziem (2016: 317) wird dies implizit herausgestellt: ,Note that LUs and constructions share basic properties. Most importantly, both are linguistic signs, that is, conventionalized pairings of form and meaning. “ Doch auch dort ist zwei Seiten später zu lesen: „Similar to frameevoking LUs, the linguistic unit evoking a construction is called a 'Construction Evoking Element' (CEE).“ (Boas, Dux \& Ziem 2016: 319). Unklar ist, ob die Betonung auf „,is called“ liegen muss. 
wickelten dreistufigen Klassifikation der Motivierung von KtE und KEE herausfallen. In diesem Abschnitt werden darüber hinaus die zuvor ausschließlich an der reflexiven Bewegungskonstruktion durchgeführten Analysen auf die reflexive Partikelverbkonstruktion ausgeweitet. Für die reflexive Weg-Konstruktion erübrigen sich entsprechende Analysen, da aufgrund ihrer geringen Produktivität (Unterabschnitt 7.5.2) für sie lediglich zwei unterschiedliche Fälle in der semantischen Motivierung ihrer Konstrukte belegt sind.

\subsection{Globale Zusammenhänge}

Die Konstruktionsgrammatik betrachtet Konstruktionen als Basiseinheiten einer Sprache: Grundlegend ist „the idea that the network of constructions captures our knowledge of language in toto - in other words, it's constructions all the way down. “ (Goldberg 2003: 223). Unbeachtet scheint allerdings die Tatsache, dass innerhalb der Frame-Semantik bereits ein ähnliches Diktum für (nicht nur sprachliches) Wissen allgemein postuliert wurde, wenn Barsalou (1992b: 40) formuliert: „Human conceptual knowledge appears to be frames all the way down. “12 Wie hängen diese beiden Ansichten zusammen? Wo ist, wenn man einen genuin konstruktionsgrammatischen und konstruktikographischen Standpunkt einnimmt, Platz für Frames? Sind Frames, wie es Barsalou andeutet, ein eigenständiges, vielleicht sogar umfassenderes Format verstehensrelevanten (sprachlichen) Wissens neben Konstruktionen? Diese Frage ist bei Überlegungen zur Verbindung beider Einheiten bisher kaum in den Blick genommen worden, was durchaus für Unklarheiten gesorgt haben mag:

So diffus sich das Verhältnis zwischen Frames und Konstruktionen auch darstellen mag und so sehr auch verschiedene konstruktionsgrammatische Theoriebildungen semantische Frames in ihr jeweiliges Modell zu integrieren versuchen - es wäre vorschnell, daraus den Schluss zu ziehen, dass Frames und Konstruktionen in einem nur zufälligen und unmotivierten Verhältnis zueinander stehen. (Ziem 2014d: 267)

Worin aber begründet sich das - angenommene - systematische und motivierte Verhältnis von Konstruktionen und Frames? Ich möchte diese Frage in zwei Schritten beantworten: einem sprachtheoretischen und einem konstruktikographischen. Deshalb zeige ich im Folgenden in Unterabschnitt 6.1.1, wie Frames in das Modell eines Konstruktikons, das nach Annahme der Konstruktionsgramma-

12 Fast identisch Barsalou (1993: 41), dort allerdings deutlicher auf die Rekursivität von Frames bezogen. 
tik das gesamte Sprachwissen strukturiert, integriert werden können und plädiere in Unterabschnitt 6.1.2 für das Primat der Semantik, das bei der empirischen Beschreibung von Konstruktionen, zuvorderst in der Konstruktikographie, implizit vorausgesetzt, aber selten explizit ausgesprochen, geschweige denn praktiziert wird. ${ }^{13}$ Diese Ideen bilden die Grundlage, um ausgehend von den globalen Zusammenhängen zwischen Konstruktionen und Frames die Parallelen zwischen ihren Strukturelementen zu verstehen.

\subsubsection{Zum Verhältnis von Konstruktionen und Frames}

Da Konstruktionen gemäß der einhelligen Auffassung aller Varianten der Konstruktionsgrammatik sowohl die Formseite als auch die semantischen Eigenschaften sprachlicher Einheiten umfassen, scheint es auf den ersten Blick unnötig, mit Frames eine zweite im sprachlichen Wissen angesiedelte Entität zu postulieren. ${ }^{14}$ Wenn also gilt, ,it's constructions all the way down“ (Goldberg 2003: 223), liegt es nicht fern, Zweifel an der sinnvollen Integrierbarkeit von Frames in diese Vorstellung anzumelden, gerade wenn es mit der analogen Formulierung von Barsalou (1992b: 40) in der Frame-Semantik eine prinzipiell konkurrierende Sichtweise gibt. Diesen Zweifeln aber liegt die Annahme zugrunde, dass Konstruktionen und Frames voneinander tendenziell unabhängige Entitäten sind. Dass dies nicht so ist, wird klar, wenn man bedenkt, „dass ein Frame die konzeptuelle Strukturgestalt der Bedeutungsseite einer Konstruktion [...] beschreibt.“ (Ziem 2008: 183). Frames und Konstruktionen sind deshalb innerhalb des sprachlichen Wissens nicht nebengeordnet, sondern Frames stehen zu Konstruktionen in einem Inklusionsverhältnis. ${ }^{15}$ Frames sind Teile von Konstruktionen, sie bilden die Grundlagen für die semantischen Eigenschaften von Konstruktionen (als

13 In der Frühphase der Konstruktionsgrammatik sind die Beziehungen zwischen syntaktischen und semantischen Strukturen unter dem Begriff des Linking thematisiert worden (z.B. Goldberg 1995: 101-119; Fillmore 1999: 115; Fried \& Östman 2004: 27). Dieser impliziert eine modularistische Trennung zwischen Syntax und Semantik und läuft einer holistischen Auffassung von Konstruktionen als ,Form-Bedeutungs-Paaren“ (Unterabschnitt 2.2.1) eigentlich zuwider (vgl. Welke 2019: 21, 71, 89).

14 Vgl. dazu Borin, Forsberg \& Lyngfelt (2013: 39): „Frames are essentially semantic units, defined by their meaning, whereas constructions are defined by their meaning and their formal structure." Eine Missinterpretation liegt allerdings darin, dass Frames keine Bedeutungen ,besitzen', sondern, wie in Unterabschnitt 4.1.1 unter Rückgriff auf das Modell von Ziem (2020b: 44-48) argumentiert, die Grundlage für Bedeutungen darstellen.

15 Schon Ziem (2008: 196) hält fest, dass „Frames [...] integrale Bestandteile von ,Konstruktionen““ sind (vgl. auch Ziem 2014b: 167). Einer solchen Auffassung entgegengesetzt argumentiert Fischer (2015: 279-281) - allerdings nicht mit dem Frame-Modell FrameNets - für eine Trennung 
Konstruktions-Frames) und ihren Konstrukten (als Konstrukt-Frames), sowohl von syntaktischen Konstruktionen wie den drei hier untersuchten Konstruktionen, als auch von lexikalischen Konstruktionen, also LE (vgl. ähnlich auch Ziem 2014c: 30). ${ }^{16}$ Hier spiegelt sich die Unterscheidung in lexikalische Frames und lexikalische Bedeutungen sowie Konstruktions-Frames und Konstruktionsbedeutungen, die ich in Kapitel 4 eingeführt habe, wider. Da abstrakte (syntaktische) Konstruktionen immer durch konkrete Konstrukte instanziiert werden, spielt ebenso die Annahme und Unterscheidung von Konstrukt-Frames und Konstruktbedeutungen eine Rolle.

Konstruktionen sind Frames übergeordnet, weil sie Formseite und semantische Eigenschaften vereinen, während Frames für sich genommen zunächst keine Aussagen über ihre formale Realisierung treffen: ${ }^{17}$,Konzeptuell unterscheiden sich Konstruktionen [...] von Frames, da es sich bei Ersteren um Zeichen handelt, die Formen und Bedeutungen verbinden.“ (Ziem, Boas \& Ruppenhofer 2014: 307). Konstruktionen ,differ from frames in having both form and meaning, a property they share with LUs“ (Bäckström, Lyngfelt \& Sköldberg 2014: 29).

In Abbildung 6.1 ist das Verhältnis zwischen Konstruktionen und Frames, wie ich es zugrunde lege, dargestellt, wobei für die semantischen Eigenschaften stellvertretend der Konstruktions-Frame oder ein relatierter lexikalischer Frame steht. Eine darüber hinausgehende Differenzierung, wie sie bei unrelatierten lexikalischen Frames notwendig ist (Unterabschnitt 5.4.2), ist hier also nicht berücksichtigt. Auf der linken Seite der Abbildung ist die Formseite der Konstruktion angegeben. Sie gibt an, wie die Strukturelemente der Konstruktion (KE, KEE und KorE) syntaktisch realisiert werden, hier in Form von Phrasentypen. ${ }^{18}$ Auf der rechten Seite ist der erwähnte Konstruktions-Frame angegeben. Beide Seiten,

zwischen Konstruktionen und Frames bei der Repräsentation situationaler Aspekte. Selbst wenn dies noch als plausibel gelten kann, ist eine solche Trennung für die semantischen Eigenschaften von Konstruktionen, die ich hier im Blick habe, nicht aufrechtzuerhalten. Entsprechend plädiert Matsumoto (2015: 308-309) dafür, auch interaktionale Frames als Bestandteile von Konstruktionen aufzufassen.

16 Mit Bezug auf die Verstehenssemantik stellt Ziem (2014d: 275) deshalb, wie schon in Unterabschnitt 2.2.3 erwähnt, die These „Verstehensrahmen strukturieren Konstruktionsbedeutungen“ auf.

17 Borin, Forsberg \& Lyngfelt (2013: 39) geben deshalb zu bedenken: „[T]he crucial difference between constructional and lexical resources is that construction entries cannot ignore the internal formal structure." Gemeint ist die formale Struktur einer Konstruktion.

18 In Abbildung 6.1 steht XP somit für eine beliebige Phrase, wobei damit in erster Linie syntaktische, phrasal realisierte Konstruktionen angesprochen sind. Für morphologische Konstruktionen kann die Realisierung der Formseite freilich anders aussehen. Zur Verwendung von Phrasentypen vgl. die Beispiele bei Fillmore, Lee-Goldman \& Rhomieux (2012: 323), Lee-Goldman \& 
Formseite und semantische Eigenschaften, bilden als Ganzes die Konstruktion, der Konstruktions-Frame ist somit Bestandteil der semantischen Eigenschaften einer Konstruktion.

Konstruktion

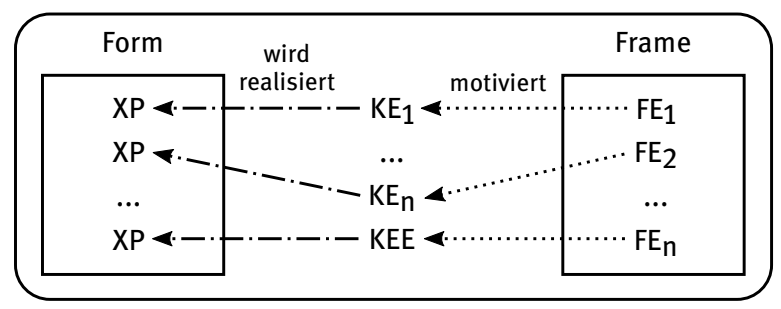

Abb. 6.1: Inklusionsverhältnis zwischen Konstruktionen und Frames

Wichtig ist nun die Tatsache, dass sowohl die Formseite der Konstruktion als auch ihr Konstruktions-Frame oder ein (relatierter) lexikalischer Frame über jeweils eigene Strukturelemente verfügen. Die Formseite besteht aus der „lexemic and/or morphosyntactic specification“ (Lee-Goldman \& Petruck 2018: 26) einer bestimmten Anzahl und Art von KE, KEE und KorE, während die semantische Seite aus FE eines entsprechenden Frames besteht. Da es sich bei dem Konstruktions-Frame um ein Bedeutungspotenzial (Unterabschnitt 4.3.1) handelt, können diese FE in Anzahl und Auswahl unterschiedlich aktiviert werden und damit in unterschiedlicher Art und Weise auf durch bestimmte Phrasentypen realisierte KtE und instanziierte KEE bezogen werden (vgl. Unterabschnitt 6.1.2). Das KorE der reflexiven Weg-Konstruktion bildet hier allerdings eine Ausnahme, da es anders als KtE (mit Ausnahme der KtE des KE EREIGNIS, vgl. Unterabschnitt 7.3.1) und KEE nicht durch FE des Konstruktions-Frames oder eines lexikalischen Frames motiviert wird, sondern selbst in der Lage ist, den Konstruktions-Frame zu evozieren (dazu Unterabschnitt 8.3.2). Aus diesem Grund ist das KorE der reflexiven Weg-Konstruktion nicht Gegenstand des vorliegenden Kapitels.

Die Strukturelemente der Konstruktion, insbesondere KE und KEE, stehen nun gewissermaßen zwischen Formseite und semantischen Eigenschaften, da sie beide in sich vereinen. ${ }^{19} \mathrm{KE}$ und KEE sind somit in der Terminologie von Langa-

Petruck (2018: 26) sowie den Überblick über mögliche Inhalte eines Konstruktionseintrags in Tabelle 7.1 in Unterabschnitt 7.1.1.

19 Vgl. dazu Borin, Forsberg \& Lyngfelt (2013: 39): ,[F]rame elements are typically semantic roles, whereas construction elements are also syntactic constituents.“ Ähnlich auch Borin et al. (2012: 12). 
cker (1987: 91) einzelne symbolische Einheiten, oder eben Konstruktionen: „CEs themselves are constructions, although these constructions may be very general.“ (Lee-Goldman \& Petruck 2018: 26). Ihre Instanzen, also KtE und die Instanzen von KEE können nun durch FE des Konstruktions-Frames und/oder eines lexikalischen Frames semantisch motiviert sein, was in Abbildung 6.1 durch die gepunkteten Pfeile auf der rechten Seite dargestellt wird. Gleichzeitig werden KtE und KEE formal durch die in der Formseite der Konstruktion festgelegten Phrasentypen realisiert. Dieses Verhältnis der Realisierung ist durch die abwechselnd gestrichelten und gepunkteten Pfeile auf der linken Seite dargestellt. KE und KEE enthalten somit gleichermaßen Informationen über ihre formale Realisierung und ihre semantischen Eigenschaften, insbesondere die mögliche Motivierung durch FE eines Frames. Diese semantischen Eigenschaften sind von konstruktikographischer Relevanz, um die Benennungen und Definitionen der KE und KEE festlegen zu können (Abschnitt 7.3), sie schaffen aber ebenso Voraussetzungen für die Evokation des Konstruktions-Frames (vgl. Unterabschnitt 8.4.2).

Das Inklusionsverhältnis zwischen Konstruktionen und Frames existiert in zwei Hinsichten, die gleichzeitig eine Gemeinsamkeit von Konstruktionen und Frames darstellen. Frames lassen sich, darauf hat schon Fillmore (1985a: 232) hingewiesen, in einem doppelten Sinne verstehen: Sie sind einerseits ein kognitives Format, also strukturierende Einheiten des verstehensrelevanten Wissens, andererseits sind sie ein Beschreibungsinstrument, das dazu dient, dieses verstehensrelevante Wissen systematisch und in Bezug auf sprachliche Äußerungen zu erfassen. Wenngleich gerade erstere Sichtweise insbesondere bei FrameNet eher implizit bleibt (vgl. Unterabschnitt 2.1.3), darf nicht vergessen werden, dass mit der linguistischen Beschreibung von Frames immer auch die Hypothese vertreten wird (oder werden sollte), dass Frames das verstehensrelevante Wissen, auf dem mithin die semantischen Eigenschaften von LE und syntaktischen Konstruktionen beruhen - ganz im Sinne der beiden Frame-Typen auf Ebene des Bedeutungspotenzials (lexikalische Frames und Konstruktions-Frames, vgl. Abschnitte 4.2 und 4.3),$-{ }^{20}$ strukturieren. Wichtig ist nun, dass für Konstruktionen grundsätzlich das Gleiche gilt:

As conventional form-meaning pairings, constructions are building blocks capable of representing both the language competence itself as well as linguistic analyses addressing

20 Interessanterweise spricht Fillmore (1985a: 232) in Bezug auf den Beschreibungsanspruch von Frames explizit von „lexical and grammatical meaning“, weist also schon hier gewissermaßen auf die Möglichkeit hin, Frames zur Beschreibung von Konstruktionen heranzuziehen (sofern man die Bezeichnung, grammatische Bedeutung ' hier nicht wörtlich versteht, vgl. Unterabschnitt 4.3.3). 
aspects of such language competence. In other words: Constructions are conceptual tools for systematically investigating a language; at the same time, they are employed to represent the knowledge that a speaker has of a language and to serve as a cognitive resource to produce and understand linguistic expressions of varying complexity. (Boas \& Ziem 2018a: 13)

Ebenso wie für Konstruktionen wird dieses doppelte Verhältnis auch am Begriff des Konstruktikons, wie er in der Konstruktikographie verwendet wird, deutlich. Auch er ist ein doppelter: Er rekurriert einerseits auf das Netzwerk von Konstruktionen, wie es als Organisationsformat des - diesmal die Formseite von Konstruktionen einbeziehenden - verstehensrelevanten Wissens verstanden wird, andererseits aber - dies ist die Lesart, die in der Konstruktikographie dominiert - auf das Ergebnis der linguistischen Beschreibung von Konstruktionen, eben die digitalen Ressourcen (vgl. Bäckström, Lyngfelt \& Sköldberg 2014: 10; Lyngfelt 2018: 2; Ziem \& Flick 2018: Abschn. 3). ${ }^{21}$ Das angesprochene Inklusionsverhältnis wird nun sowohl in der kognitivistischen als auch in der konstruktikographischen Lesart wirksam: Frames strukturieren einerseits das verstehensrelevante Wissen auf Seite der semantischen Eigenschaften von Konstruktionen, andererseits sind sie praktisches Beschreibungsformat für diese. Für die Konstruktikographie gilt also wie für die Hypothese des mentalen Konstruktikons: Frames sind Teile von Konstruktionen und ihren Beschreibungen, sie sind ihnen nicht nebengeordnet. Oder, allgemeiner gefasst: „,[G]rammar is not distinct from semantics, but rather incorporates semantics as one of its two poles.“ (Langacker 2009b: 1).22 Ohne das Inklusionsverhältnis von Konstruktionen und Frames klar auszusprechen, reformulieren Blyth \& Koike (2014: 105) Goldbergs Maxime der „constructions all the way down“ (Goldberg 2003: 223) und vereinen sie - ohne diese explizit zu erwähnen - mit dem eingangs zitierten Standpunkt von Barsalou (1992b: 40). Mit Blick auf satz- und turn-übergreifende Strukturen gilt für sie: „[I]t’s constructions (plus frames) all the way up.“ (Blyth \& Koike 2014: 105).

21 Herbst (2019: 5) spricht ausgehend von klassischer Lexikographie von den zwei Lesarten als mentalem Konstruktikon und Referenz-Konstruktikon. Daneben wirft Herbst (2016: 172) die Frage auf, ob ein beschreibungspraktisches (Referenz-)Konstruktikon das Spiegelbild eines mentalen Konstruktikons sein muss oder kann und beantwortet sie aufgrund mangelnder kognitionsempirischer Fundierung negativ. Der naheliegendste Grund für die nötige Beschränkung des Umfangs eines beschreibungspraktischen Konstruktikons aber liegt in der forschungspraktischen Umsetzbarkeit, worauf Herbst (2019: 6) später selbst hinweist. Indes machen Bäckström, Lyngfelt \& Sköldberg (2014: 10) deutlich, dass das mentale Konstruktikon eher Gegenstand der Konstruktionsgrammatik ist, während ein beschreibungspraktisches Referenz-Konstruktikon Ziel der Konstruktikographie ist.

22 Vgl. auch die ähnliche Formulierung in Langacker (2005a: 104). 
Konkret für das oben skizzierte Inklusionsverhältnis von Konstruktionen und Frames bedeutet dies, dass eine Konstruktion einerseits kognitionstheoretisch als Inklusion einer Formseite und eines Frames betrachtet werden muss und andererseits ihre Beschreibung beide Seiten berücksichtigen muss. Auf die Strukturparallelen, um die es in diesem Kapitel geht, bezogen, heißt das sowohl, dass KE und KEE als kognitive Einheiten des mentalen Konstruktikons Informationen über ihre formale Realisierung und die mögliche semantische Motivierung ihrer KtE durch FE lexikalischer Frames und eines Konstruktions-Frames enthalten müssen, als auch, dass die konstruktikographische Beschreibung beide Seiten miteinander vereinen muss, gerade um die komplexen semantischen Voraussetzungen, die mit der Instanziierung einer Konstruktion und der Evokation eines KonstruktionsFrames einhergehen, erfassen zu können.

Aus all diesen Gründen ist die schlichte Gegenüberstellung von Konstruktionen und Frames in Tabelle 6.1 unzutreffend. In letzter Konsequenz bedeutet dies, dass die vorrangigen Einheiten der Beschreibung des verstehensrelevanten Wissens Konstruktionen sein müssen, ${ }^{23}$ Frames werden dann als (ein wesentlicher) Teil ihrer Beschreibung relevant.

Was für die jeweiligen Untersuchungsgegenstände - Konstruktionen und Frames - gilt, gilt demnach auch für die beiden Theorien: Konstruktionsgrammatik zu betreiben, hieße dann, gleichzeitig auch Frame-Semantik zu betreiben. ${ }^{24}$ Ein Ausspielen der beiden Theorien gegeneinander, wie Welke (2019, 2021a) es - zugunsten der Konstruktionsgrammatik - tut, halte ich aus konstruktionssemantischer Sicht für unnötig. ${ }^{25}$

23 Allgemein hat dies bereits Nemoto (1998: 240) erkannt: „Armed with frame semantics, Construction Grammar is expected to encompass ever-wider arrays of data.“ Konkret auf die kognitionslinguistische Leistung bezogen ist die Einschätzung von Fried \& Östman (2004: 18): „Construction Grammar has the potential for a uniform representation of all grammatical knowledge.“ In dieser Perspektive müsste es allerdings nicht allein um ,grammatisches' Wissen gehen, sondern um verstehensrelevantes Wissen generell, da ja Konstruktionen als Ganzes gerade kein rein grammatisches, sondern ebenso ein semantisches Phänomen sind.

24 Daneben erwägen Östman \& Fried (2005) noch den umgekehrten Fall der Vorstellung von der Konstruktionsgrammatik als Teil der Frame-Semantik, lassen diese Frage aber offen.

25 Mehr noch: Eine um die Frame-Semantik reduzierte Konstruktionsgrammatik gegen holistische sprachtheoretische Modelle in Stellung zu bringen, wie Welke (2019: 48-50, 2021a) es vorschwebt, widerspricht auf ganzer Breite dem Kerngedanken der Konstruktionsgrammatik. Wohin eine solche Auffassung von Grammatik (und Sprache generell) führen soll, bleibt völlig unklar jedenfalls, wenn ein konstruktionsgrammatischer Ansatz das Ziel sein soll. Wohl auch deshalb schafft Welke (2019: 54) es nicht, einen Holismus in allen Aspekten (vor allem hinsichtlich der definitorischen Einheit von Form und semantischen Eigenschaften in einer Konstruktion) abzulehnen. 
In der allgemeinen Wahrnehmung erscheinen KxG und Frame-Semantik jedoch oft als ,unentwirrbar“ (Ziem 2014[d]: 263). Das gilt [sic!] sowohl von Befürwortern der KxG als auch von Kritikern aus. ${ }^{[\ldots]}$ Ich betrachte die allgemeine Frame-Semantik weder als identisch mit der $\mathrm{KxG}$ noch als Bestandteil einer $\mathrm{KxG}^{[\ldots]}$ und plädiere daher für eine Unterscheidung von KxG und allgemeiner Frame-Theorie (vgl. 5.4.1). (Welke 2019: 49)26

Ein solches ,Splitting، von Theorien (im Sinne von Goldberg 2009: 219) wäre einerseits zwar im Sinne von Ockhams Rasiermesser. Bevor die Konstruktionsgrammatik andererseits aber nicht in der Lage ist, semantische Zusammenhänge in einem einheitlichen Format ohne Einbezug der Frame-Semantik zu beschreiben, halte ich Welkes Programmatik für verfehlt. Gerade im Zusammenspiel hätte eine um die Frame-Semantik angereicherte Konstruktionsgrammatik und Konstruktikographie erst dann wirklich die Fähigkeit, den Anspruch einer kognitiven Sprachtheorie durchzusetzen. ${ }^{27}$ So hält Fischer (2008) in Bezug auf Fillmores Verstehenssemantik fest:

Die Konstruktionsgrammatik in Verbindung mit dem Fillmoreschen Semantikkonzept versteht sich daher als umfassende Sprachtheorie ohne Sicherheitsnetz: keine die Verwendung bedingende Information kann in andere Bereiche der Sprachbeschreibung abgeschoben werden. Stattdessen umfassen die Aufgaben, die eine konstruktionsgrammatische Beschreibung leisten muss, die Erfassung der subtilen Unterschiede in der Verwendung von verschiedenen Konstruktionen sowie die Repräsentation des semantischen, pragmatischen und Weltwissens, das die Verwendung der jeweiligen Konstruktionen bestimmt. (Fischer 2008: 81-82)

Allerdings ist unbestritten, dass eine Sprachtheorie aus Konstruktionsgrammatik und Frame-Semantik, wie Fischer (2008) sie hypothetisch entwirft, bisher noch nicht ausentwickelt wurde. Etwas weniger hypothetisch, allerdings stärker auf kognitive Fragestellungen bezogen, formulieren es lakonisch Östman \& Fried (2005: 8): „Construction Grammar, together with Frame Semantics, has the potential for addressing cognitive issues.“ Bezogen auf interaktionale Frames drückt es Matsumoto (2015: 310) wie folgt aus: „Knowledge of grammar should [...] be modeled as a partnership of grammatical constructions with interactional frames“.

26 Die Annahme der ,Unentwirrbarkeit‘ beider Theorien, die Welke Ziem zurechnet, bezieht sich jedoch nur auf deren Provenienz, nicht auf die Theorien an sich: ,Dass zwischen Frame-Semantik und Konstruktionsgrammatik eine enge Beziehung besteht, dürfte dabei außer Frage stehen; sie werden vielfach schon deswegen als ,Schwestertheorien" verstanden, weil beide unentwirrbar mit dem Namen Charles Fillmore verbunden sind.“ (Ziem 2014d: 263).

27 Die Frage, ob die Konstruktionsgrammatik eine kognitive Theorie ist, wird allerdings über ihre einzelnen Varianten (Abschnitt 2.2) hinweg unterschiedlich beantwortet, wobei insbesondere die formal ausgerichteten Ansätze der Berkeley und Sign-Based Construction Grammar keinen solchen Anspruch verfolgen (vgl. dazu Ziem \& Lasch 2013: 77). 


\subsubsection{Semantische Motivierung von Konstrukten}

Die Annahme, dass Frames als Grundlage der semantischen Eigenschaften von Konstruktionen und Konstrukten als Teile dieser zu verstehen sind, beantwortet noch immer nicht die Frage, welcher Teil einer Konstruktion nun sowohl theoretisch als auch konstruktikographisch als primär aufzufassen ist, mit anderen Worten: Richtet sich die Form einer Konstruktion nach ihren semantischen Eigenschaften oder die semantischen Eigenschaften nach ihrer Form? Kann man, wie Welke (2019: 36), tatsächlich von einem „Primat der Form“ ausgehen (vgl. auch Welke 2011: 13-14, 2021a: 409-410)? Am Beispiel der reflexiven Bewegungskonstruktion lässt sich diese Frage etwa im Hinblick auf das KEE konkretisieren, genauer: hinsichtlich der Kookkurrenz des Reflexivums mit LE, die eigentlich keine ,reflexiven' Verben sind. ${ }^{28}$ Wie ist es beispielsweise zu erklären, dass Verben wie arbeiten, kämpfen oder wünschen, die außerhalb von Konstrukten dieser Konstruktion kaum als ,reflexive Verben zu klassifizieren wären, in den Belegen (1)(3) mit einem Reflexivum auftreten?

(1) Seitdem arbeitet sich der Professor acht Monate im Jahr mit einem Jeep durch die Wüste von Turkmenistan, harrt in Kasachstan stundenlang in ungeheizten Sitzungszimmern aus und bewahrt in Usbekistan auch nach dem sechsten Wodka noch die Contenance. (Die Zeit, 24.02.2000, Nr. 9)

(2) David kämpfte sich durch das überfüllte Lokal und reduzierte seinen Wortschatz auf: Moment, gleich, Sekunde, bin schon da und sofort. (Suter, Martin: Lila, Lila, Zürich: Diogenes 2004, S. 52)

(3) Doch an diesem Morgen im Parlamentssaal der Mongolei wünscht sich Knieper einen Moment lang in den deutschen Wissenschaftsbetrieb zurück, wo man Meinungsverschiedenheiten in Fachzeitschriften austrägt. (Die Zeit, 24.02.2000, Nr. 9)

28 Vgl. dazu speziell bereits die entsprechende Beobachtung von Kühnhold (1973: 184, 283) zur reflexiven Partikelverbkonstruktion. Nur für Verben, die außerhalb der reflexiven Bewegungskonstruktion distributionell nicht frequent mit einem Reflexivum kookkurrieren, kann deshalb behauptet werden, dass sich ihr Auftreten mit einem Reflexivums „nur konfigurationell ableiten lässt“ (Âgel 1997a: 181). Allerdings würden Verben, die auch sonst als ,reflexiv‘ gelten, einen grundsätzlich anderen Status innerhalb einer Reflexivkonstruktion wie der reflexiven Bewegungskonstruktion haben. Statt diesem distributionellen Kriterium ziehe ich ein semantisches vor, nämlich das der Frage nach dem (relatierten oder unrelatierten) lexikalischen Frame, den das Verb evoziert (dazu Unterabschnitt 5.4.2). Diese Unterscheidung spielt deshalb insbesondere in den Abschnitten 6.2 und 6.3 eine weiterhin wichtige Rolle. 
Die Antwort hierauf kann nur eine semantische sein. Belege wie (1)-(3) legen den Schluss nahe, dass sich, zumindest für Fälle, die denen der reflxiven Bewegungskonstruktion ähnlich sind, die Form der Konstruktion, insbesondere die Kombination aus einem Reflexivum mit einem KE, das LE wie arbeiten, kämpfen oder wünschen als KtE erlaubt, nach ihren semantischen Eigenschaften richten muss. Genauer: nach dem zu erzielenden Konstrukt-Frame.

Aus diesem Grund möchte ich für alle weiteren Überlegungen in diesem Kapitel die Annahme zugrunde legen, dass Konstruktionen und insbesondere ihre Teile - KE und deren KtE sowie ebenso KEE - semantisch motiviert sind. ${ }^{29}$ Damit ist gemeint, dass z.B. das Auftreten eines syntaktischen Elements, das andernfalls nicht zu erwarten wäre, im Wesentlichen auf semantische Ursprünge in der Konstitution eines spezifischen Konstrukt-Frames zurückgeht. ${ }^{30}$ Hierzu zählt bei den drei hier untersuchten Konstruktionen deren jeweiliges KEE, also das Reflexivum, in nicht wenigen Fällen aber auch das KtE des KE Weg bzw. 〈WEG〉, z.B. eines wie durch das überfüllte Lokal in (2), das allein durch den lexikalischen Frame Hostile_encounter, den die LE kämpfen evoziert, nicht zu erklären ist, also nicht als Instanz eines seiner FE gelten kann. In diesem Fall muss das KtE durch das überfüllte Lokal anders erklärt werden: Es ist nicht wie etwa das KtE David, das das FE Hostile_encounter.SIDE_1 instanziiert, durch ein FE des lexikalischen Frames Hostile_encounter semantisch motiviert, sondern durch eines des Konstruktions-Frames Motion. Mit anderen Worten: Es findet eine FrameAnpassung des lexikalischen Frames durch den Konstruktions-Frame (Unterabschnitt 4.4.1) statt. Die Form der Konstruktion und ihrer Konstrukte ist somit wesentlich durch semantische Anforderungen bestimmt: „[S]emantic structure determines or, better, motivates grammatical structure.“ (Croft 2001: 108). ${ }^{31}$

29 Vgl. dazu die Ideen von Lasch (2018a: 100, 2020) zu einem semantisch motivierten Konstruktikon. Lasch (2020: 137) spricht programmatisch von Konstruktionen als „Bedeutungs-FormPaaren" statt der in der Konstruktionsgrammatik sonst zu findenden Redeweise von ,FormBedeutungs-Paaren‘ (z.B. Lakoff 1987: 467; Goldberg 1995: 4).

30 Aus übersetzungswissenschaftlicher Perspektive plädiert Čulo (2013) und Czulo (2017, 2020) unter dem Begriff des primacy of frame model ebenso für ein Primat semantischer Aspekte, sieht allerdings Konstruktionen - anders als ich es oben skizziert habe - nicht als integratives Format aus einer Formseite und einem Frame an.

31 Diese Annahme ist freilich Teil einer kontroversen Diskussion und auch für den umgekehrten Fall gibt es Evidenz (vgl. für einen Überblick Croft 2001: 108-110). Vom Standpunkt einer inhärent symbolischen Grammatikkonzeption, für die auch ich in Unterabschnitt 6.1.1 plädiert habe, gibt Langacker (2005a: 105) Folgendes zu bedenken: „On this view, grammar (or grammatical form) does not symbolize semantic structure, but rather incorporates it, as one of its two poles. If grammar is wholly reducible to assemblies of symbolic structures, it is incoherent to say that some aspect of grammar functions as the symbolizing element in such assemblies.“ 
Den Begriff der Motivierung verwende ich im Anschluss an das Postulat der Ikonizität syntaktischer Strukturen von Haiman (1980, 1983, 1985). Grundlegend hierfür ist die Annahme einer Diagrammatizität von Sprache, mit anderen Worten: „[L]anguages are like diagrams.“ (Haiman 1985: 9). Motivierung in diesem Sinne ist definiert als ,a perceived similarity between the structure of a diagram and the structure of the concepts that it represents.“ (Haiman 1985: 71). Strukturparallelen zwischen Konstruktionen und Frames lassen sich auf Token-Ebene als Korrespondenzen zwischen KtE und KEE einerseits und FE andererseits auffassen. Ein KtE oder KEE kann damit durch ein oder mehrere FE motiviert sein. Haimans Definition von Motivierung lässt sich damit gleichsam auf das Konstrukt als Ganzes beziehen: Das Konstrukt - das Diagramm - ist durch eine konzeptuelle Struktur - einen oder mehrere Frame(s) - motiviert. Wie Goldberg (2005a) schreibt, ist die (semantische) Motivierung von Konstrukten und Konstruktionen ein entscheidender Motor für die Herausbildung einer Konstruktion als ,Form-Bedeutungs-Paar‘:

[T] he type of Construction Grammar adopted here demands that motivation be sought for each construction that is posited. Motivation aims to explain why it is at least possible and at best natural that this particular form-meaning correspondence should exist in a given language. (Goldberg 2005a: 17)

Folgerichtig weist Goldberg (2005a: 38) darauf hin, dass die Motivierung von Konstruktionen (man muss auch sagen: Konstrukten) auf bestimmte kommunikative Funktionen (hier: eines Bezeichnungsbedarfs) zurückzuführen ist und eine Annahme von Arbitrarität nicht mehr rechtfertigt. ${ }^{32}$ Da Konstruktions-Frames, wie ich in Abschnitt 4.4 gezeigt habe, Blends aus einem lexikalischen Frame und einem Konstruktions-Frame sein können und eine Konstruktbedeutung durch den ihr zugrunde liegenden Konstrukt-Frame grundsätzlich analysierbar bleibt, bietet sich der Begriff der Motivierung gerade für die Erklärung eines Konstrukt-Frames an: „Analyzable expressions are not built from their component expressions. Rather, they are motivated by these expressions." (Coulson 2001: 48). ${ }^{33}$ Bezogen auf

32 An anderer Stelle weist sie unter anderem auf Ikonizität als Motor für die Motivierung einer Konstruktion hin: „Motivation can be provided by (for example) appeal to constraints on acquisition, principles of grammaticalization, discourse demands, iconic principles, or general principles of categorization.“ (Goldberg 2005b: 216).

33 In einem ähnlichen Kontext spricht auch Langacker (1997: 11) von Motivierung, wenn er festhält, dass Konstitutenten (konstruktikographisch: KtE) weniger einzelne „Bausteine“ einer größeren Struktur (konstruktikographisch: einem Konstrukt) sind, sondern dass sie der Kategorisierung einzelner Einheiten dieser Struktur dienen. In diesem Zusammenhang betont er ebenso, dass Konstituenten abhängig von einer Konstruktion sind und nicht umgekehrt. Mit dem vorliegenden Ansatz lässt sich diese Auffassung wie folgt in Einklang bringen: KtE und KEE sind 
die syntaktische Form eines Konstrukts lässt sich mit Welke (2005: 37) festhalten: „Formalsyntaktisches ist der Reflex von Semantischem.“

Für das eben diskutierte Beispiel der Kookkurrenz des Reflexivums mit Verben, die ansonsten nicht als ,reflexiv` zu klassifizieren sind, lässt sich auf folgende Annahme zurückgreifen: „The linguistic separateness of an expression corresponds to the conceptual independence of the object or event which it represents." (Haiman 1983: 783). Die empirische Tatsache, dass sich LE wie die oben genannten in Konstrukten der reflexiven Bewegungskonstruktion wiederfinden, lässt einen ikonischen Schluss auf die semantische Motivierung der Konstrukte zu: Das ansonsten nicht obligatorische Reflexivum zeigt an, dass neben dem (unrelatierten) lexikalischen Frame eine weitere Komponente, eben der Konstruktions-Frame, im Spiel ist und eine Frame-Anpassung des lexikalischen Frames stattfindet. Die für LE wie arbeiten, kämpfen oder wünschen eigentlich nicht vorgesehene Argumentstruktur ist somit ein ikonischer Hinweis auf einen von einem (unrelatierten) lexikalischen Frame deutlich unterscheidbaren, separaten Konstruktions-Frame (vgl. auch Hampe \& Schönefeld 2003: 246-247):

[T] he unusual, quasi-borrowed argument structure serves as a (diagrammatic) iconic clue to the intended interpretation in that it triggers the retrieval of at least one other verbal concept, more typically associated with the respective argument structure and fitting the contextual requirements. The intended meaning was hypothetically proposed to be arrived at through the conceptual integration/blending of the two verbal concepts thus activated. (Hampe \& Schönefeld 2006: 128)

Ein zusätzliches, in der Argumentstruktur eigentlich nicht erwartbares Element wie ein Reflexivum kann also bereits ein Hinweis auf ein zusätzliches, durch einen Blending-Prozess (Unterabschnitt 4.1.3) integriertes Konzept und damit eine Frame-Anpassung sein: „One possible indicator of blending is the occurrence of idiosyncratic combinations of syntactic categories.“ (Barlow 2000: 326). ${ }^{34}$ So kommt Haiman (1983: 795-799, 1985: 143) durch einen typologischen Vergleich zwischen Sprachen, die Reflexivität durch ein syntaktisch separates Reflexivum erzeugen, mit solchen, die anstelle dessen eine morphologische Inkorporation aufweisen, zu dem Schluss, dass Erstere einen Hinweis auf die Erweiterung des zugrunde liegenden Konzepts (also: eine Frame-Anpassung) zulassen, Letztere jedoch nicht: „A separate word denotes a separate entity; a bound morpheme does not.“ (Haiman 1983: 795). Ist ein separates Reflexivum statt einer morpho-

nicht atomar, sondern durch ihre semantische Motivierung im Hinblick auf das gesamte Konstrukt (und damit den korrespondierenden Konstrukt-Frame) definiert.

34 Auch Fauconnier (1997: 31) weist auf die Fähigkeit von Reflexiva hin, spezifische Verbindungen (mappings) in mental spaces herzustellen. 
logischen Inkorporation im Spiel, kann dies also ein Hinweis auf ein weiteres Konzept sein: „Linguistic fusion signals conceptual fusion; linguistic independence signals conceptual independence.“ (Haiman 1985: 142). Mehr noch: Im Falle der reflexiven Bewegungskonstruktion und der reflexiven Partikelverbkonstruktion spielt die Kombination des KEE mit einem KtE des KE EREIGNIS unter bestimmten Umständen eine entscheidende Rolle bei der Evokation des Konstruktions-Frames, weshalb dem KEE eine entsprechend hohe syntagmatische Kombinationsrelevanz zukommt (dazu Unterabschnitt 8.3.3).

Für die semantische Motivierung der Konstrukte der drei untersuchten Konstruktionen heißt das, dass sich das Auftreten des Reflexivums in manchen Konstrukten direkt durch den Bezeichnungsbedarf des FE Motion.THEme des Konstruktions-Frames ergibt, insbesondere bei Verben, die üblicherweise nicht ,reflexiv" sind und unrelatierte lexikalische Frames evozieren. ${ }^{35}$ Gleiches gilt für die KtE des KE WEG bzw. 〈WEG〉. In bestimmten Fällen sind sie Resultat des Bezeichnungsbedarfs der FE Motion.Source, Motion.PATH, Motion.Goal oder Motion.Direction des Konstruktions-Frames, gerade dann, wenn ein solches FE nicht im lexikalischen Frame, den das KtE des KE EREIGNIS evoziert, angelegt ist. Allgemeiner formuliert: Die gesamte Konstellation von KE und ihre instanziierten KtE stehen ikonisch für den Bedarf an FE, die sprachlich ausgedrückt werden müssen, wie auch Croft (2001) feststellt:

Why is syntactic structure mostly iconic? Because an iconic mapping between function and form is one of the easiest ways to allow a hearer to identify the semantic components corresponding to the syntactic elements of a construction. (Croft 2001: 236)

In diesem Sinne ist die Form eines Konstrukts nicht primär, sondern durch den zugrunde liegenden Konstrukt-Frame determiniert und strukturiert: So ist „die formalsyntaktische Struktur [...] als eine Funktion der semantisch-pragmatischen Struktur“ (Welke 2005: 70) zu verstehen. Die gesamte Konstruktion und jedes ihrer Konstrukte sind vollständig semantisch motiviert. Diese Motivierung kann sich aus zwei Quellen speisen: dem lexikalischen Frame und/oder dem KonstruktionsFrame. Beide können bei der Motivierung einzelner KtE und KEE auch gemeinsam agieren, indem sie KtE abwechselnd oder sogar doppelt motivieren (vgl. die Varianten einer Frame-Anpassung in Unterabschnitt 4.4.1). Es deutet sich also

35 Es handelt sich in diesen Fällen mit Welke (2005: 80) formuliert um eine Analyse von „formalsyntaktischen Strukturen, die nicht nur Folge sind, sondern auch Zweck, die also die Funktion bekommen (vom Sprecher mit der Intention geäußert werden), eben dieses SemantischPragmatische auch zu signalisieren. Oder vom Hörer aus gesagt: Formalsyntaktisches kann die bloße Folge von Semantisch-Pragmatischem sein, kann aber auch für den Hörer Kennzeichen von Semantisch-Pragmatischem sein oder werden.“ 
an, dass die Art und Weise der semantischen Motivierung eines Konstrukts und dessen KtE variieren kann, je nachdem, welchen Anteil lexikalischer Frame und Konstruktions-Frame jeweils am Konstrukt-Frame haben. Diese Varianten der Motivierung möchte ich im Folgenden in den Blick nehmen. Die Blickrichtung dabei ist im Einklang mit der Konstruktikographie eine onomasiologische: Sie fragt ausgehend von semantischen Eigenschaften von Konstruktionen und KE und KEE nach deren formaler Realisierung. ${ }^{36}$ Im Vordergrund steht dabei stets die Konstitution eines Konstrukt-Frames, denn sie gibt Auskunft darüber, durch welche FE die KtE und KEE des Konstrukts motiviert werden, in welchem Verhältnis also lexikalische Frames und Konstruktions-Frame jeweils stehen.

In den folgenden drei Abschnitten 6.2 bis 6.4 nehme ich unter dem Gesichtspunkt der semantischen Motivierung von Konstrukten und ihren KtE und KEE die reflexive Bewegungskonstruktion in den Blick. Jene ist der reflexiven WegKonstruktion vorzuziehen, da sie ihr gegenüber über eine wesentlich höhere Produktivität verfügt (vgl. Unterabschnitt 7.5.2) und damit eine größere Bandbreite an Konstrukt-Frames aufweist. Die reflexive Partikelverbkonstruktion bietet demgegenüber Anlass zur Betrachtung im Rahmen einiger Sonderfälle in der semantischen Motivierung von Konstrukten, weshalb ich auf sie erst in Abschnitt 6.4 zurückkomme.

Obwohl die semantische Motivierung, wie ich zeigen werde, von Konstrukt zu Konstrukt variiert, ist es möglich, die allgemeinen Zusammenhänge schematisch zu skizzieren. Bevor ich in Kapitel 7 methodische Wege zur konstruktikographischen Generalisierung der Analysen auf Konstruktebene vorschlage, sei bereits an dieser Stelle mit Abbildung 6.2 eine exemplarisch auf die reflexive Bewegungskonstruktion konkretisierte Darstellung des Inklusionsverhältnisses zwischen ihrer Formseite und ihrem Konstruktions-Frame Motion vorgestellt.

Auf der linken Seite sind die typischen syntaktischen Realisierungen der KE und des KEE in Phrasentypen angegeben. ${ }^{37}$ Die rechte Seite enthält die Kern-FE des Frames Motion und zeigt die Motivierung der KE bzw. deren KtE sowie der Instanzen des KEE stellvertretend durch diesen prototypischsten aller relatierten lexikalischen Frames (der zugleich der Konstruktions-Frame ist, vgl. Unterabschnitt 5.4.2) an: Die KtE des KE BEWEGENDES werden in diesem prototypischen Fall (d.h.:

$\overline{36}$ Noch allgemeiner sieht Finkbeiner (2018: 156) konstruktionistische Ansätze grundsätzlich als onomasiologisch (d.h., nicht-kompositionell verfahrend) an, während projektionistische semasiologisch (kompositionell) vorgingen.

37 Eine Ausnahme stellt das Reflexivum REFL dar: Ich verzichte der Übersichtlichkeit halber darauf, es (wie andere Pronomen auch, vgl. Duden 2016: 808-809) als NP zu klassifizieren und setze stattdessen eine separate Kategorie an. Dies gilt auch für die Angabe der beteiligten Phrasentypen in den Konstruktionseinträgen im Zusatzmaterial. 
Reflexive Bewegungskonstruktion

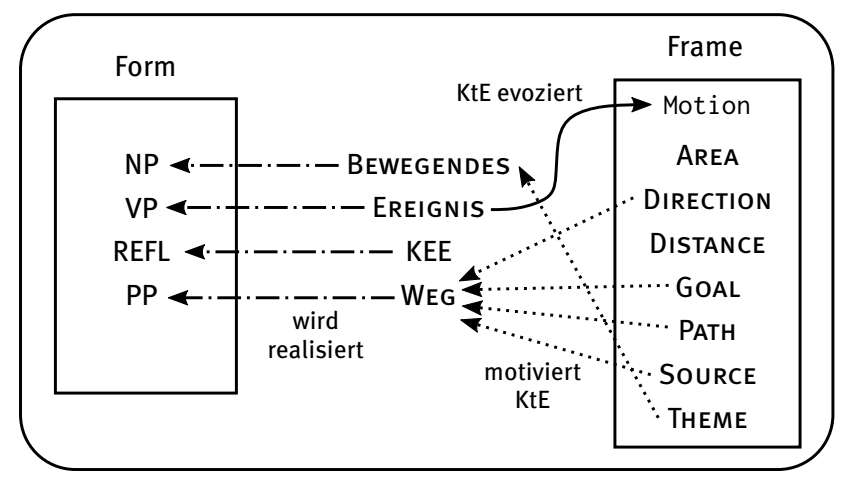

Abb. 6.2: Inklusionsverhältnis zwischen der reflexiven Bewegungskonstruktion und ihrem Konstruktions-Frame Motion

der Identität von lexikalischem Frame und Konstruktions-Frame) durch das FE Mot ion.THEME motiviert. Die KtE des KE Weg können durch die FE Mot ion.Source, Motion.PATH, Motion.GoAL oder Motion.DIRECTION motiviert werden, wobei, wie ich in Unterabschnitt 6.4.1 aufzeige, auch eine mehrfache Instanziierung dieses KE möglich ist, bei der mehrere dieser FE zugleich in mehreren KtE realisiert werden. Aufgrund der von Konstrukt zu Konstrukt variierenden Motivierung der KtE dieses KE zeigen gepunktete Pfeile von mehreren FE auf ein einzelnes KE. Auffällig ist, dass für die prototypische Motivierung eines Konstrukts der reflexiven Bewegungskonstruktion kein FE zur Verfügung stellt, um das KEE zu motivieren. Dies ist zuvorderst auf eine Besonderheit des für das Englische definierten Frames Motion zurückzuführen, auf die ich in Unterabschnitt 6.3.1 zurückkomme. Schließlich ist ebenso kein FE für das KE EREIGNIS vorgesehen, da dieses stets durch die LE instanziiert wird, die den lexikalischen Frame evoziert (vgl. Abschnitt 4.2), weshalb es auch bei der konstruktikographischen Benennung und Definition der Strukturelemente gesondert behandelt werden muss (vgl. Unterabschnitt 7.3.1). Dabei ist es möglich, dass dieses KE durch eine LE instanziiert wird, die direkt den Konstruktions-Frame Motion als lexikalischem Frame evoziert, weshalb in Abbildung 6.2 ein Pfeil von diesem KE auf den Namen des Frames verweist.

Diese skizzierten semantischen Motivierungsverhältnisse mit dem Auftreten von Motion als lexikalischem Frame stellen lediglich den prototypischsten Fall dar (vgl. dazu Unterabschnitt 5.4.2). Daneben existiert eine ganze Reihe von Fällen, für die diese prototypische Motivierung nicht gilt, gerade wenn lexikalischer Frame und Konstruktions-Frame nicht identisch sind und auch nicht in Frame- 
Nähe zueinander stehen und somit eine Frame-Anpassung stattfindet. Auf die unterschiedlichen Ausprägungen dieser Motivierungsverhältnisse möchte ich, stets beginnend mit den Fällen relatierter lexikalischer Frames, in den nächsten beiden Abschnitten eingehen.

\subsection{Konstruktelemente und Frame-Elemente}

Die Gegenüberstellung von KtE und FE erscheint als basalste Strukturparallele zwischen Konstruktionen und Frames jenseits eines globalen Bezugs. Sowohl Konstruktionen und ihre Konstrukte als auch Frames sind strukturierte Einheiten und so liegt es nahe, dass das Verstehen einer Konstruktion die, wie Croft (2001: 234) vermutet, Wahrnehmung einer „correspondence between the elements [einer Konstruktion, A.W.] and the components of its semantic structure“ beinhaltet. Konkret werden die Parallelen zwischen KtE und FE spätestens seit dem Beginn konstruktikographischer Bemühungen postuliert. So begründen Fillmore, Lee-Goldman \& Rhomieux (2012: 322, Anm. 20) ihre Definition von KtE explizit mit deren semantischer Motivierung durch FE: „For temporary technical reasons we called these constituents 'construct elements' rather than frame elements in our annotation; but conceptually these are frame element labels." Eine Annahme wie diese wird häufiger erwähnt, meist allerdings aus einem theoretischen und programmatischen Blickwinkel, weniger als Ergebnis empirischer Analysen (z.B. Shead 2011: 175; Ziem 2014c: 30). Stärker empirisch geht bisher allein das Konstruktikon für das Brasilianische Portugiesisch vor, indem es - wie in Unterabschnitt 2.3.2 erwähnt - KE rein formal definiert und ihre semantischen Eigenschaften durch FE repräsentiert (vgl. Torrent et al. 2014: 35, 44; Laviola et al. 2017: 194-195).

Die Idee, die KtE eines Konstrukts und - in einem zweiten Schritt - auch die KE der Konstruktion mit FE eines Frames zu parallelisieren, basiert auf zwei Grundannahmen. Die erste Grundannahme besagt, dass es möglich sein muss, die semantischen Eigenschaften einer LE, die als KtE eines KE (im Falle der drei untersuchten Konstruktionen: des KE EREIGNIS) instanziiert wird, sowie die semantischen Eigenschaften der Konstruktion, genau wie ihre Formseite in kleinere Teile zu zerlegen. Dies ist dadurch gegeben, dass beide Typen semantischer Eigenschaften auf Frames beruhen (Abschnitte 4.2 und 4.3), deren innere Struktur durch FE repräsentiert wird. Diese FE können auf die einzelnen Teile eines Konstrukts, die KtE und Instanzen von KEE, und generalisiert auch auf die einzelnen Teile der Konstruktion, KE und KEE, bezogen werden (vgl. zu Letzterem Abschnitt 7.3). Sowohl die Konstruktion als auch lexikalische Frames und ein Konstruktions-Frame 
sind dabei die obersten Organisationseinheiten, während die Zerlegung in ihre Teile spezifisch für das Konstrukt ist (vgl. auch Croft 2005: 287):

[T] he whole construction is the primitive unit of representation and the parts are derivative. Thus, the meaning of the whole construction, [...], is basic and the analysis into parts, [...] is derived from the meaning of the whole and the breakdown of the whole into parts. (Croft 2001: 183)

FrameNet wird dieser Annahme gerecht, indem es die Bestandteile eines Frames von ihm ableitet: FE sind, wie in Unterabschnitt 2.1.2 erwähnt, stets framespezifisch definiert und erheben keinen Anspruch auf eine universalgrammatische Gültigkeit, noch nicht einmal eine Gültigkeit über Frames hinweg. Eine analoge Annahme für Konstruktionen wird allerdings nur in der Radical Construction Grammar postuliert, nämlich, „that syntactic categories are constructionspecific.“ (Croft 2001: 108).

Die zweite Annahme besagt, dass die semantischen Eigenschaften eines Konstrukts bis zu einem gewissen Grad ,kompositionell‘ erklärt werden können. Dies ist im konstruktionssemantischen Modell angelegt: Da sich die Unterscheidung von Frames und Bedeutungen, die ich in Unterabschnitt 4.1.1 erläutert habe, auch auf Token-Ebene und damit auf Konstrukte überträgt, liegt einer Konstruktbedeutung stets ein Konstrukt-Frame zugrunde, der selbst wenn er durch einen Blend aus lexikalischem Frame und Konstruktions-Frame zustande kommt, als Addition von FE dieser beiden Frames verstanden werden kann (Unterabschnitt 4.1.3). Diese FE eines Konstrukt-Frames können dann wiederum auf die Strukturelemente eines Konstrukts bezogen werden. Indes zeigen sogar Analysen von Phrasemen, also Konstrukten, die als Paradefall von Nicht-Kompositionalität gelten dürften (vgl. aus konstruktionsgrammatischer Sicht Ziem \& Lasch 2013: 10-11), dass sie mit Hilfe einer strikten Parallelisierung ihrer Bestandteile (KtE bzw. KEE) mit entsprechenden semantischen Bestandteilen (also etwa: FE) gewissermaßen ,kompositionell' $\mathrm{zu}$ erfassen sind (vgl. Croft 2001: 179-185). ${ }^{38}$

Um die Zusammenhänge an einem Beispiel zu verdeutlichen, sei das Konstrukt in (4) herangezogen. In ihm sind alle KtE (außer das des KE EREIGNIS, vgl. Unterabschnitt 7.3.1) entweder durch FE des lexikalischen Frames Daring (im Falle von Mehr und mehr französische Politiker) oder durch FE des KonstruktionsFrames (sich und in Unterhaltungssendungen) semantisch motiviert. Der Kon-

38 Ein ähnliches Modell entwickelt Finkbeiner (2006), allerdings ohne direkte Bezüge zwischen syntaktischer Form und semantischen Bestandteilen herzustellen. Bei grundsätzlichen Fragen der Zerlegbarkeit der Bedeutung von Phrasemen weist sie auch auf frame-semantische Ansätze hin (vgl. Finkbeiner 2006: 140). 
strukt-Frame besteht dann aus der Summe von FE beider Frames (vgl. die Analyse in Unterabschnitt 4.4.2).

(4) [agent Mehr und mehr französische Politiker] [Daring wagen] [sich ${ }^{\text {THEME}}{ }^{\text {] }}$ [in Unterhaltungssendungen GoAL]. (Die Zeit, 27.04.2000, Nr. 18)

Natürlich ist damit aber nicht gesagt, dass auf der Ebene einer Konstruktbedeutung keine nicht-kompositionellen Bestandteile existieren können. Der Standardwert der ,Schwierigkeit‘, den ich für die reflexive Bewegungskonstruktion stellvertretend für alle drei untersuchten Konstruktionen in Unterabschnitt 5.7.2 analysiert habe, ist ein Beispiel dafür. Dieses Phänomen der Standardwerte in Konstruktbedeutungen, die nicht zwingend auf eine lexikalische Bedeutung oder die Konstruktionsbedeutung zurückgeführt werden müssen, lässt sich in Termini der konzeptuellen Integration mit dem Begriff der emergenten Struktur erfassen, die den Gegenstand eines eigenen semantischen Parameters bildet (Abschnitt 5.7) und auch für die konstruktikographische Beschreibung einer Konstruktion eine Rolle spielt (Abschnitt 7.6).

Bisherige konstruktikographische Bemühungen um die Parallelisierung von KtE und FE, wie sie sich etwa in der im Eingang zu diesem Kapitel kritisierten Gegenüberstellung in Tabelle 6.1 niederschlagen, müssen vor dem Hintergrund der semantischen Motivierung von KtE mit Vorsicht betrachtet werden. Zum einen, weil die formalen Eigenschaften von KtE als Schlussfolgerung aus der Annahme der semantischen Motivierung von Konstruktionen ebendieser Motivierung gegenüber sekundär sind (vgl. Unterabschnitt 6.1.2). Zum anderen, weil keineswegs stets eine Eins-zu-eins-Übereinstimmung zwischen KtE und FE existiert und deshalb auch keine Eins-zu-eins-Übereinstimmung von KE und FE postuliert werden kann.

Zuvorderst empirisch untersucht werden muss, welchem Frame die FE angehören, die ein KtE motivieren - ob sie also dem lexikalischen Frame oder dem Konstruktions-Frame (oder beiden gemeinsam) zuzuordnen sind. Dies kann nur eine Analyse auf KtE-Ebene, also auf Token-Ebene leisten. Da es aber letztlich um die semantische Beschreibung der Konstruktion auf Type-Ebene geht, müssen die Ergebnisse einer solchen Analyse im Anschluss generalisiert werden (Abschnitt 7.3). Die im Rahmen der Konstruktikographie bisweilen suggerierte Annahme, dass die semantischen Motivierungen von KE einfach gerichtet sind und auch die KtE stets durch FE - und zwar immer dieselben FE - eines einzigen Frames, sei es des lexikalischen Frames oder des Konstruktions-Frames, motiviert sind (vgl. meine Kritik zu Beginn dieses Kapitels), muss in Zweifel gezogen werden. Mit anderen Worten: Eine Unterscheidung der beiden Typen von Frames und Bedeutungen auf Type-Ebene (lexikalischer Frame bzw. lexikalische Bedeutung 
und Konstruktions-Frame bzw. Konstruktionsbedeutung) wird hierbei ebenso vernachlässigt wie die Trennung zwischen Type- und Token-Ebene allgemein. Ich möchte in diesem Abschnitt deshalb zeigen, dass die Untersuchung der Strukturparallelen zwischen Konstruktionen und Frames auf Token-Ebene und damit auf Ebene von Konstrukten und der Konstitution von Konstrukt-Frames stattfinden muss. Dies trägt dem Umstand Rechnung, dass sich die Strukturparallelen zwischen KtE - nicht KE - und FE je nach dem lexikalischen Frame, der in einen Konstrukt-Frame eingeht, unterschiedlich gestalten.

Berücksichtigt man, dass an der Konstitution eines Konstrukt-Frames sowohl ein lexikalischer Frame allein als auch lexikalischer Frame und KonstruktionsFrame zugleich beteiligt sein können (vgl. Unterabschnitt 4.1.3), ergeben sich für die semantische Motivierung von KtE drei Varianten. ${ }^{39}$

1. Alle KtE eines Konstrukts können vollständig durch FE des lexikalischen Frames motiviert sein (Unterabschnitt 6.2.1).

2. Ein einzelnes KtE eines Konstrukts kann durch ein FE des KonstruktionsFrames motiviert sein (Unterabschnitt 6.2.2).

3. Ein einzelnes KtE eines Konstrukts kann zugleich durch ein FE des lexikalischen Frames und ein FE des Konstruktions-Frames motiviert sein (Unterabschnitt 6.2.3).

Für den Fall, dass in die Motivierung aller KtE oder eines einzelnen KtE nur ein Frame-Typ involviert ist, spreche ich im Folgenden von einfacher Motivierung. Fälle, in denen ein KtE durch FE beider Frame-Typen zugleich motiviert wird, bezeichne ich als doppelte Motivierung. In allen Fällen der einfachen Motivierung eines einzelnen KtE durch den Konstruktions-Frame und der doppelten Motivierung findet eine Frame-Anpassung des lexikalischen Frames durch den Konstruktions-Frame statt. Mit anderen Worten: Die letzten beiden der drei Vari-

39 Welke (2019: 197, 203, 2021a: 378) unterscheidet derweil drei Arten des Zusammenspiels von (projektionistischer) lexikalischer Bedeutung und (konstruktionistischer) Konstruktionsbedeutung: (1) Die vollständige Übereinstimmung von Projektion und Konstruktion, (2) die NichtÜbereinstimmung von Konstruktion und Projektion, deren Ergebnis Koerzionseffekte sind, und (3) die ausgeschlossene Übereinstimmung von Projektion und Konstruktion, die keine Instanziierungen hervorbringt und zu ungrammatischen Konstrukten führen würde. In den vorliegenden Überlegungen entspricht (1) der einfachen Motivierung aller KtE durch den lexikalischen Frame. Welkes Fall (2) differenziere ich hingegen weiter aus, indem ich sowohl die einfache Motivierung eines KtE durch FE des Konstruktions-Frames (und nicht des lexikalischen Frames) als auch die doppelte Motivierung eines KtE durch FE beider Frames in Betracht ziehe. Welkes Fall (3) steht aus gebrauchsbasierter (korpuslinguistischer) Perspektive zunächst nicht zur Diskussion: Auf die Blockierung ,ungrammatischer‘ Konstrukte gehe ich nicht ein. 
anten drücken aus, dass ein Konstrukt-Frame als Blend aus lexikalischem Frame und Konstruktions-Frame konstituiert wird.

Ich möchte die drei Varianten im Folgenden exemplarisch für die reflexive Bewegungskonstruktion aufzeigen. Ich beginne mit solchen Fällen, in denen ein relatierter lexikalischer Frame für die Motivierung aller KtE verantwortlich ist, in denen also nur jener lexikalischer Frame allein (der im Falle von Motion mit dem Konstruktions-Frame identisch ist) daran beteiligt ist (Unterabschnitt 6.2.1). Im Anschluss daran untersuche ich für unrelatierte lexikalische Frames schrittweise die Rolle des Konstruktions-Frames bei der semantischen Motivierung der KtE, die in der einfachen Motivierung eines einzelnen KtE durch den Konstruktions-Frame (Unterabschnitt 6.2.2) oder der doppelten Motivierung gemeinsam mit einem FE des lexikalischen Frames (Unterabschnitt 6.2.3) bestehen kann. Da das Phänomen der doppelten Motivierung eine Auseinandersetzung mit dem von Goldberg (1995: 50-52) formulierten Semantic Coherence Principle nahelegt, möchte ich dies in Unterabschnitt 6.2.4 kritisch betrachten.

\subsubsection{Einfache Motivierung aller KtE durch lexikalischen Frame}

Der einfachste Fall der semantischen Motivierung von KtE liegt vor, wenn diese vollständig durch FE des lexikalischen Frames motiviert werden, und zwar ausschließlich durch solche. Einfach ist dieser Fall deshalb, weil ein separater Konstruktions-Frame keinen Anteil an der semantischen Motivierung der KtE und damit an der Konstitution des Konstrukt-Frames hat. Es findet also keine Frame-Anpassung statt. Die Annahme, dass „[f]ür die Gesamtbedeutung eines Konstrukts [...] weder nur die Konstruktion noch die jeweiligen Lexeme alleine verantwortlich sein“ (Albert 2015: 537) können, trifft also nur auf Konstrukte $\mathrm{zu}$, deren Konstrukt-Frame aus lexikalischem Frame und Konstruktions-Frame gleichermaßen konstitutiert ist. Für Konstrukte, deren KtE sämtlich durch den lexikalischen Frame einfach motiviert sind, gilt sie nicht.

Untersucht man die Konstitution des Konstrukt-Frames als semantische Motivierung der KtE durch einen der beiden Frame-Typen - einen lexikalischen Frame oder den Konstruktions-Frame -, wird deutlich, dass der Konstrukt-Frame sehr wohl allein durch den lexikalischen Frame konstituiert werden kann. Dafür muss allerdings eine Voraussetzung gegeben sein: Der lexikalische Frame muss mit dem Konstruktions-Frame identisch sein oder zu ihm in Frame-Nähe stehen. Es muss sich also gemäß der Einteilung in relatierte und unrelatierte Frames (Unterabschnitt 5.4.2) um einen relatierten lexikalischen Frame handeln. Ist dieser lexikalische Frame gar identisch mit dem Konstruktions-Frame, gilt: „[T]he semantics associated with these lexical items is redundant with the semantics of 
the construction.“ (Goldberg 1992: 48). Diese einfache Motivierung aller KtE durch den lexikalischen Frame betrifft alle Konstrukte, in denen ein relatierter lexikalischer Frame evoziert wird, in denen der Konstrukt-Frame also allein aus FE dieses Frames besteht. Mit anderen Worten: Ein relatierter lexikalischer Frame (und damit auch der Konstruktions-Frame selbst) ist stets in der Lage, alle KtE eines Konstrukts vollständig semantisch zu motivieren. ${ }^{40}$

Die LE, die diese lexikalischen Frames evozieren - in der reflexiven Bewegungskonstruktion, der reflexiven Partikelverbkonstruktion und der reflexiven Weg-Konstruktion also die KtE des jeweiligen KE EREIGNIS -, sind somit Ausgangspunkt einer „elaboration of the meaning of the construction.“ (Goldberg 1997: 386). ${ }^{41}$ Wird das KE EREIGNIS also durch ein KtE instanziiert, das als LE eines relatierten lexikalischen Frames gilt und werden dadurch alle KtE der Konstruktion durch FE von deren lexikalischem Frame motiviert, ist der Konstrukt-Frame am deutlichsten kompositionell aufgebaut (vgl. ähnlich Welke 2019: 29).42 Bei den drei hier untersuchten Konstruktionen liegt dieser Fall bei KtE des KE EREIGNIS vor, die entweder Motion selbst evozieren oder einen Frame, der zu ihm in einer Frame-Nähe steht, kurzum: einen beliebigen zu Motion relatierten Frame. Nur bei einer Identität von lexikalischem Frame und Konstruktions-Frame kann allerdings unter Rückgriff auf die Auffassung der semantischen Eigenschaften einer Konstruktion als ,Beitrag' $z u$ denjenigen eines Konstrukts (vgl. Unterabschnitt 4.3.2) davon gesprochen werden, dass ,the contribution of the construction is wholly redundant with the meaning of the verb." (Bencini \& Goldberg 2000: 642). Von einer Elaboration im engeren Sinne kann erst dann gesprochen werden, wenn

40 Aus valenztheoretischer Perspektive trifft das Valency Realisation Principle von Herbst (2014: 200) eine vergleichbare Aussage: „Valency Realisation Principle: if a valency construction of a verb is fused with an argument structure construction and all of its participant roles are construed as argument roles, then the formal realisation of the argument structure construction (SYN) must coincide with the valency pattern of the valency construction.“

$41 \mathrm{Zu}$ dieser Relation der ,Elaboration' fügt Goldberg (1997: 396) noch ,kraftdynamische Relationen' (zu denen ,means', ,Instrument‘, ,Resultat‘ und ,Ablehnung‘ gehören), ,Vorbedingung‘ sowie ,kookkurrierende Aktivität‘ hinzu. Bei Goldberg (1998: 46-49) kommen zu ,Elaboration‘ noch ,means', ,Instrument‘, ,Resultat', ,Vorbedingung', ,Negation‘ und ,kookkurrierende Aktivität‘ (mit anderen Worten: ,manner') hinzu. Vgl. Stefanowitsch (2008b) für Argumente, diesen Relationen größere Beachtung zu schenken. Empirische Evidenz für die von Goldberg (1997: 396) formulierte Hierarchie zwischen solchen Relationen zeigt Rohde (2001: 107-116) auf.

42 Hampe \& Schönefeld (2003: 254) weisen darauf hin, dass es bei der Elaboration einer ,Konstruktionsbedeutung d durch eine lexikalische Bedeutung keinen Unterschied macht, ob die ,Konstruktbedeutung ' direkt durch eine, im Hintergrund‘ wirkende ,Konstruktionsbedeutung zustande kommt (vgl. unten) oder ob eine konzeptuelle Integration im Spiel ist. Es ist deshalb davon auszugehen, dass bei Fällen, in denen der Konstrukt-Frame vollständig durch einen lexikalischen Frame konstituiert wird, kein Blending-Prozess vorliegt (vgl. Unterabschnitt 4.1.3). 
es sich um einen lexikalischen Frame handelt, der in einer Frame-Nähe von +1 oder niedriger bzw. -1 oder niedriger zum Konstruktions-Frame steht.

Die Frames, die für die drei untersuchten Konstruktionen hierfür infrage kommen, habe ich bereits samt der jeweiligen Anzahl ihrer Konstrukte nach Framezu-Frame-Relationen zu Motion geordnet in den Tabellen 5.12 bis 5.17 in Unterabschnitt 5.4.3 als System der Frame-Nähen von Motion zusammengestellt. In Tabelle 6.2 sind diese Ergebnisse noch einmal in aggregierter Form zusammengefasst, geordnet nach Anzahl der Konstrukte (Kx) für jeden relatierten lexikalischen Frame, sofern dieser für die reflexive Bewegungskonstruktion (RBKxn), die reflexive Partikelverbkonstruktion (RPVKxn) oder die reflexive Weg-Konstruktion (WegKxn) belegt ist.

Tab. 6.2: Konstrukte der reflexiven Bewegungskonstruktion mit einfacher Motivierung aller KtE durch lexikalische Frames

\begin{tabular}{|c|c|c|c|c|c|c|}
\hline \multirow[b]{2}{*}{ Lexikalischer Frame } & \multicolumn{2}{|c|}{ RBKxn } & \multicolumn{2}{|c|}{ RPVKxn } & \multicolumn{2}{|c|}{ Weg-Kxn } \\
\hline & Kx & Anteil & Kx & Anteil & Kx & Anteil \\
\hline Body_movement & 246 & $34,55 \%$ & 118 & $38,82 \%$ & & \\
\hline Self_motion & 159 & $22,33 \%$ & 56 & $18,42 \%$ & & \\
\hline Motion & 105 & $14,75 \%$ & 24 & $7,89 \%$ & 26 & $100,00 \%$ \\
\hline Cause_motion & 77 & $10,81 \%$ & 53 & $17,43 \%$ & & \\
\hline Change_direction & 43 & $6,04 \%$ & 17 & $5,59 \%$ & & \\
\hline Bringing & 17 & $2,39 \%$ & 8 & $2,63 \%$ & & \\
\hline Fluidic_motion & 17 & $2,39 \%$ & & & & \\
\hline Placing & 14 & $1,97 \%$ & 11 & $3,62 \%$ & & \\
\hline Motion_directional & 11 & $1,54 \%$ & 15 & $4,93 \%$ & & \\
\hline Evading & 7 & $0,98 \%$ & & & & \\
\hline Departing & 6 & $0,84 \%$ & & & & \\
\hline Fleeing & 5 & $0,70 \%$ & & & & \\
\hline Mass_motion & 2 & $0,28 \%$ & & & & \\
\hline Making_faces & 1 & $0,14 \%$ & & & & \\
\hline Ride_vehicle & 1 & $0,14 \%$ & & & & \\
\hline Undressing & 1 & $0,14 \%$ & & & & \\
\hline Excreting & & & 1 & $0,33 \%$ & & \\
\hline Operate_vehicle & & & 1 & $0,33 \%$ & & \\
\hline Gesamt & 712 & $100,00 \%$ & 304 & $100,00 \%$ & 26 & $100,00 \%$ \\
\hline
\end{tabular}

Am Beispiel der lexikalischen Frames Motion, Departing und Bringing sei nun exemplarisch an der reflexiven Bewegungskonstruktion demonstriert, wie sich die einfache Motivierung aller KtE durch einen lexikalischen Frame in unterschiedlichen Varianten vollzieht. 
Im Falle von Motion werden die KtE des KE BEwEGENDEs durch das FE Motion.Theme motiviert. Da das KtE des KE EREIGNIS durch die LE instanziiert wird, die den lexikalischen Frame evoziert, wird es nicht durch FE dieses Frames oder des Konstruktions-Frames motiviert (dazu weiterhin Unterabschnitt 7.3.1). Dies gilt für alle Konstrukte aller drei Konstruktionen und auch für solche, in denen andere Motivierungsverhältnisse wie das hier diskutierte vorliegen. Ein KtE des KE WEG hingegen kann innerhalb eines lexikalischen Frames, anders als ein KtE des KE BEWEGENDES, durch unterschiedliche FE motiviert werden. Im Falle von Motion sind Motion.Source, Motion.PATH, Motion.GoAL oder Motion.DiRECTION möglich (dazu auch Unterabschnitte 6.4.1 und 7.3.3). Welches dieser FE in einem Konstrukt auftritt, hängt freilich zu einem großen Teil von der Präposition ab, die den Kopf des KtE des KE WEG bildet. ${ }^{43}$ Die folgenden Belege sind Beispiele für das mit Abstand am häufigsten belegte FE Motion.GoAL (dazu auch Unterabschnitt 6.3.2) und die LE begeben (go.v) in (5), bewegen (move.v) in (6), rücken (move.v) in (7), verschieben (move.v) in (8) und winden (wind.v) in (9).

(5) a. Ich befand mich gerade in München und [Motion begab] mich [GoAL in ein Geschäft, das überaus luxuriös wirkte, wie ein Gourmettempel]. (Die Zeit, 10.02.2000, Nr. 7)

b. Sofort begibt sich [Theme ihr junger Begleiter von RTL] [GoAL an ihre Seite]. (Die Zeit, 27.01.2000, Nr. 5)

c. JOHANNES PAUL reiste nach Madaba und [Motion begab] sich [GoAL auf den Berg Nebo, wo nach der Überlieferung der Bibel Moses das gelobte Land erschaut haben soll]. (Archiv der Gegenwart, 2001 [2000])

d. Dr. Hans Wilhelm Stein, Burgherr von Saaleck, verbarg die beiden und [Motion begab] sich [GoAL nach München], in Ehrhardts Hauptquartier, um falsche Pässe und einen Fluchtwagen zu organisieren. (Die Zeit, 30.03.2000, Nr. 14)

e. Oder aber [THemE Sie] [Motion begeben] sich [GoAL unter die Langschläfer], denn in den Halbschlafphasen am späteren Morgen wird auch viel geträumt. (Die Zeit, 20.04.2000, Nr. 17)

f. Am 10. Oktober [Motion begab] sich [Theme TSVANGIRAI] freiwillig [GoAL zu einem polizeilichen Verhör in Harare]. (Archiv der Gegenwart, 2001 [2000])

43 Für den vorliegenden Zusammenhang ist die quantitative Verteilung der FE, die die KtE des KE WEG motivieren, von weniger großer Relevanz, da sie von einem lexikalischen Frame zum anderen variieren. Interessanter ist sie für den Fall, in dem das KtE des KE WEG allein einfach durch ein FE des Konstruktions-Frames motiviert wird, während der lexikalische Frame unrelatiert ist. Auf diese Fälle komme ich in Unterabschnitt 6.2.2 zurück. 
(6) a. Doch [Theme $\mathrm{er}$ [Motion bewegte] sich [GoAL auf sie] zu und würde ihr bald winken mit seinem dritten, aus dem breitschultrigen Rücken herauswachsenden Arm und sie so sehr damit meinen, daß sie nicht anders konnte als zurückzuwinken hinter dem Fenster, von dem sie nun wußte, daß er sie dort sehen konnte und nur sie dort sah. (Düffel, John von: Vom Wasser, München: dtv 2006, S. 167)

b. [Tнеме Der Rücken vor ihr, in einem dicken, warmen, grauen Mantel], [Motion bewegte] sich [GoAL zu einem Bahnsteig], Malka folgte ihm. (Pressler, Mirjam: Malka Mai, Weinheim Basel: Beltz \& Gelberg 2001, S. 246)

(7) [Theme Er] [Motion rückt] sich [GoAL in den Mittelpunkt]. (Schwanitz, Dietrich: Männer, Frankfurt a. M.: Eichborn 2001, S. 73)

(8) [Tнеме Der Schwerpunkt des Westens] [Motion verschob] sich [GoAL in die USA]. (Die Zeit, 02.03.2000, Nr. 10)

(9) [Theme Jonas] [Motion wand] sich aus dem Kofferraum nach vorne [GoAL auf die Rückbank des Autos]. (Glavinic, Thomas: Die Arbeit der Nacht, München Wien: Carl Hanser Verlag 2006, S. 346)

Die folgenden Belege sind Beispiele für die Motivierung der KtE des KE WEG durch das FE Motion.PATH mit LE wie begeben (go.v) in (10), bewegen (move.v) in (11), mäandern (meander.v) in (12), schlängeln (snake.v) in (13) und winden (wind.v) in (14).

(10) Das „wahrscheinlich“ gründete sich dabei ausschließlich auf das Vertrauen in die Überzeugungskraft seiner eigenen Argumente, die er am gleichen Tag Bismarck in einem Schreiben und am folgenden Tag dem Kaiser in einem ebensolchen vortrug, in dem er diesen zugleich um eine Audienz für einen Bevollmächtigten bat - [Theme er selber] [Motion begab] sich in diesen Tagen „auf ärztlichen Rat“ [РАтн Krupp, Berlin: Siedler 2000, S. 174)

(11) a. [THEмE Er] [Motion bewegte] sich [РАTH durch vermeintlich unauffällige harmonische Räume] mit einer Sehnsucht nach Farbigkeit, die alle Grauwerte des Theoretischen löschte. (Die Zeit, 10.02.2000, Nr. 7)

b. Über der Eingangstür [Motion bewegt] sich [Theme die DM] im schwerelosen Raum [РАтн um unseren globalisierten Planeten]. (Die Zeit, 17.02.2000, Nr. 8)

c. [Theme Die von Beduinen geführte Karawane (bis zu 18 Teilnehmer)] [Motion bewegt] sich in täglichen Etappen von vier bis sechs Stunden [РАTH 
bei die Kamele als Reit- und Packtiere fungieren. (Die Zeit, 17.02.2000, Nr. 8)

(12) So [Motion mäandern] sich [THEME die nackten und verschlungenen Leiber] [РАтн durch die Werbung und über die Titel jener der Werbeästhetik folgenden Magazine]. (Die Zeit, 13.01.2000, Nr. 3)

(13) a. Der Schmerz kam wieder und [Motion schlängelte] sich [РАтH durch den wunden Körper]. (Dölling, Beate: Hör auf zu trommeln, Herz, Weinheim: Beltz \& Gelberg 2003, S. 66)

b. Bald [Motion schlängelten] sich [Tнеме so viele Kabel] durch die Wiese und [РАтн über den Betonboden], daß er alle paar Meter strauchelte. (Glavinic, Thomas: Die Arbeit der Nacht, München Wien: Carl Hanser Verlag 2006, S. 379)

c. Zu beiden Seiten [Motion schlängelten] sich [Theme Blumenbeete] [РАтн um schmale Rasenstreifen]. (Düffel, John von: Houwelandt, Köln: DuMont Literatur und Kunst Verlag 2004, S. 175)

(14) Er trägt den Bauchnabel immer frei und [Motion windet] sich wie eine Schlange [РАтн um gefeierte Designer]. (Die Zeit, 02.03.2000, Nr. 10)

Deutlich weniger zahlreich als Konstrukte, in denen ein KtE des KE WEG durch das FE Motion.GoAL oder Motion.PATH motiviert wird, sind solche, in denen dies die FE Motion.Source oder Motion.Direction übernehmen. Die folgenden Belege sind Beispiele für erstere Variante und die LE begeben (go.v) in (15), bewegen (move.v) in (16), rühren (move.v) in (17), schlängeln (snake.v) in (18), ringeln und winden (wind.v) in (19).

(15) [Theme HASSAN] [Motion begab] sich Mitte Oktober in Begleitung einer tausend Mann starken bewaffneten Eskorte [Source von Djibouti aus] nach Mogadischu, um seine Macht von dort aus auszuüben. (Archiv der Gegenwart, 2001 [2000])

(16) Ein Drahtseil ist über den Fluß gespannt, mit einer beweglichen Winde ist das Fährboot daran festgetäut, und während die gewaltige Strömung auf das querstehende Ruder drückt, [Motion bewegen] sich [Theme Boot und Winde] seitlich am Seil entlang [Source von einem Ufer] zum andern. (Düffel, John von: Vom Wasser, München: dtv 2006, S. 192)

(17) [Theme Vater und Tochter] [Motion rührten] sich nicht [Source von ihren Stühlen] und schienen doch einander nähergerückt. (Hahn, Ulla: Unscharfe Bilder, München: Deutsche Verlags-Anstalt 2003, S. 275) 
(18) Ringeln sich etwa nicht [Theme ein paar Schlangen] um sie herum im Sand oder in ihrem Nacken, [Motion schlängeln] sich herab [Source von ihrer Brust]. (Venske, Regula: Marthes Vision, Frankfurt am Main: Eichborn Verlag 2006, S. 128)

(19) Ich bekam ihren Arm zu fassen, aber [Theme sie] [Motion wand] sich [Source aus meinem Griff]. (Franck, Julia: Lagerfeuer, Köln: DuMont Literatur und Kunst Verlag 2003, S. 53)

Schließlich ist das FE Motion.DIRECTION für Konstrukte mit der LE bewegen (move.v) belegt, wie in (20) zu sehen.

(20) [Tнеме Die augenblickliche Wirtschaftslage] [Motion bewege] sich [Direction in Richtung Armut], was das Resultat der ausbeuterischen Privatisierung sei. (Archiv der Gegenwart, 2001 [2000])

Belege wie diejenigen in (5)-(20) zeigen die prototypischsten Konstrukte der reflexiven Bewegungskonstruktion, da ihr lexikalischer Frame jeweils direkt dem Konstruktions-Frame entspricht (vgl. dazu Unterabschnitt 5.4.2). Ein Blending von lexikalischem Frame und Konstruktions-Frame und damit eine FrameAnpassung finden bei der Konstitution des Konstrukt-Frames (Unterabschnitte 4.1.3 und 4.4.2) also nicht statt, da es sich um einen relatierten lexikalischen Frame handelt. Den Schluss, dass Fälle wie diese prototypisch sind, lässt die aus Tabelle 6.2 ersichtliche hohe Frequenz entsprechender Konstrukte zu. Aber auch aus theoretischer Sicht scheint es nicht überraschend, dass lexikalische Frames, die mit dem Konstruktions-Frame identisch sind oder in Frame-Nähe zu ihm liegen, wie in Unterabschnitt 5.4.2 definiert, prototypisch sind:

It is clear that the most common and prototypical case is one in which the verb and the construction do not designate two separate events. Rather the verb designates the same event that the construction designates, or the verb elaborates the constructional meaning. (Goldberg 2010: 53)

Dies könnte zu einer von Boas (2008a: 132) für die Caused-Motion-Konstruktion (Goldberg 1995: 152-179) vertretenen Annahme verleiten, die reflexive Bewegungskonstruktion könnte als Abstraktion aller Konstrukte verstanden werden, deren lexikalischer Frame dem Konstruktions-Frame entspricht und die somit die zur Motivierung aller KtE notwendigen FE bereits enthält. ${ }^{44}$ Diese Abstraktion ist aller-

44 Für die LE, die diese relatierten lexikalischen Frames evozieren, lässt sich also mit Kunze (1997: 135) festhalten, „daß diese Bewegungsverben bereits als Basisverben ein Direktional zu sich nehmen können und daß sie (eher eine Ausnahme) zusätzlich die gerade behandelte Modi- 
dings nicht allein auf den einen lexikalischen Frame, der mit dem KonstruktionsFrame identisch ist, zurückzuführen. Bei dieser Identität ist sie am offensichtlichsten, doch auch andere lexikalische Frames als der mit dem KonstruktionsFrame identische sind für die einfache Motivierung aller KtE möglich. Lediglich eine Voraussetzung müssen diese lexikalischen Frame erfüllen: Sie müssen in Frame-Nähe zum angenommenen Konstruktions-Frame stehen, also relatierte lexikalische Frames sein.

Alle KtE können auch dann durch einen lexikalischen Frame einfach motiviert werden, wenn dieser einem anderen als dem Konstruktions-Frame entspricht: Alle relatierten lexikalischen Frames kommen hierfür infrage. Für jeden dieser Frames liegt dann eine „elaboration“ (Goldberg 1997: 386) des Konstruktions-Frames vor, wobei ,the event type designated by the verb is an instance of the more general event type designated by the construction." (Goldberg 1995: 60). Die Höhe dieser Elaboration nimmt mit abnehmender Frame-Nähe des lexikalischen Frames zu. Wie Tabelle 6.2 zeigt, sind in den Daten für die reflexive Bewegungskonstruktion lexikalische Frames belegt, die in vier verschiedenen Frame-zu-Frame-Relationen zu Motion stehen: ${ }^{45}$

- Vererbungsrelation: Fluidic_motion (+1), Mass_motion $(+1)$, Motion_directional (+1), Self_motion (+1), Fleeing (+2);

- Benutzt-Relation: Body_movement $(+1)$, Bringing $(+1)$, Change_direction $(+1)$, Departing (+1), Evading (+1), Placing (+1), Undressing (+2), Making_faces $(+3)$;

- Kausativ-Relation: Cause_motion $(-1)$;

- Siehe_auch-Relation: Bringing (+1), Ride_vehicle (+1), Self_motion (+1).

Die Prinzipien der Motivierung aller KtE durch den lexikalischen Frame Motion, die ich oben illustriert habe, übertragen sich nun auf alle weiteren relatierten lexikalischen Frames. Am ähnlichsten sind sie noch bei den innerhalb der Vererbungsrelation in einer Frame-Nähe von $+1 \mathrm{zu}$ Motion stehenden Frames Fluidic_motion, Mass_motion, Motion_directional und Self_motion mit überwiegend ähnlichen FE, insbesondere denen, die für die Motivierung der KtE des KE BEwEGENDES verantwortlich sind. Grund dafür ist das in Unterabschnitt 5.4.3 angesprochene wesentliche Kennzeichen der Vererbungsrelation als „strongest relation between frames, corresponding to is-a in many ontologies“ (Ruppenhofer et al. 2016: 80): Der untergeordnete Frame verfügt über alle seman-

fikation [durch Hinzunahme eines (fakultativen) Reflexivums, A.W.] erlauben, womit dann keine Bedeutungsveränderung zu einem Bewegungsverb mehr verbunden ist.“

45 Zur leichteren Übersicht wiederhole ich in der folgenden Diskussion der einzelnen Relationen ihre bereits in Unterabschnitt 5.4.3 erwähnten Definitionen. 
tischen Eigenschaften des übergeordneten Frames entweder in derselben oder einer höheren Spezifiziertheit (vgl. Ruppenhofer et al. 2016: 80). Für den lexikalischen Frame Self_motion, der in meinen Daten unter den in Vererbungsrelation zu Motion relatierten Frames mit Abstand am frequentesten vertreten ist, betrifft dies etwa das FE Self_motion.SELF_MOVER, das als spezifischeres Korrelat des FE Motion.Theme zu verstehen ist. ${ }^{46}$ Bei Fluidic_motion ist das Korrelat Fluidic_motion.FLUID, bei Mass_motion wird das FE als Mass_motion.MASS_THEME spezifiziert, wohingegen bei Motion_directional eine nicht erkennbare Spezifizierung auf das FE Motion_directional.THEME vorliegt. Die FE Motion.Source, Motion.Path, Motion.Goal und Motion.Direction hingegen werden von Self_motion und den anderen Frames in Vererbungsrelation zu Motion mit denselben Namen und teilweiser Spezifizierung in der Definition entsprechend geerbt.

Um die Motivierung aller KtE durch einen relatierten lexikalischen Frame noch an weiteren Beispielen zu illustrieren, seien zwei Frames, die in einer anderen Frame-zu-Frame-Relation zu Motion stehen, nämlich der Benutzt-Relation und der Siehe_auch-Relation, ausgewählt: Departing und Bringing. Auf die Kausativ-Relation und Cause_motion als lexikalischen Frame gehe ich in Unterabschnitt 6.3.1 bei der Diskussion um die Motivierung des KEE ein.

Departing steht innerhalb der Benutzt-Relation in einer Frame-Nähe von +1 zu Motion. Die Benutzt-Relation als ,one of the largest frame-to-frame relations in FrameNet“ (Sikos \& Padó 2018: 43) besagt, wie bereits in Unterabschnitt 5.4.3 erwähnt, dass ,, a particular frame makes reference in a very general kind of way to the structure of a more abstract, schematic frame." (Ruppenhofer et al. 2016: 83). Jene Verbindung zu einem übergeordneten Frame besteht indes ,almost exclusively for cases in which a part of the scene evoked by the child [dem untergeordneten Frame, A.W.] refers to the parent frame [dem übergeordneten Frame, A.W.]“ (Ruppenhofer et al. 2016: 83).

Von den 33 Frames, die in einer Benutzt-Relation zu Motion stehen (16 in einer Frame-Nähe von $+1,12$ in einer Frame-Nähe von +2 und 5 in einer Frame-Nähe von +3 , vgl. Tabelle 5.13 in Unterabschnitt 5.4.3), sind in den Daten für die reflexive Bewegungskonstruktion 8 belegt, zu denen Departing gehört. Konstrukte mit Departing als lexikalischem Frame sind für die LE ausklinken und entfernen (beide leave.v) belegt, wie die Belege unter (21) und (22) zeigen.

46 Dies lässt sich über die zu den Frame-zu-Frame-Relationen analog ausgestalteten FEzu-FE-Relationen anschaulich im FrameNet-FrameGrapher (https://framenet.icsi.berkeley.edu/ fndrupal/FrameGrapher, zuletzt abgerufen am 07.09.2021) sichtbar machen, welcher allerdings die Daten auf FrameNets Website und nicht die Version des Daten-Release 1.7 dokumentiert. 
(21) [Theme Kim Tang] [Departing klinkt] sich noch vor Mitternacht [Source aus dem Gelage] aus, um am nächsten Morgen fit am Arbeitsplatz zu erscheinen. (Die Zeit, 23.03.2000, Nr. 13)

(22) a. Anspar Klein berichtete von seinen Besuchen in Bruchsal und daß er im Laufe der Jahre mehr und mehr den Eindruck gehabt habe, [Тнеме Hans Arbogast] [Departing entferne] sich [SourcE aus der Realität]. (Hettche, Thomas: Der Fall Arbogast, Köln: DuMont Buchverlag, 2001, S. 248)

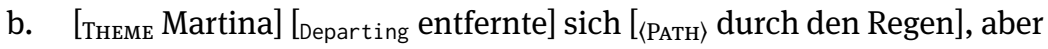
ihre Haare wurden nicht naß, während sie endlich ging. (Kuckart, Judith: Lenas Liebe, Köln: DuMont Literatur und Kunst Verlag 2002, S. 262)

c. Durch des Mannes ruckartige Bewegungen verunsichert, nähert sich Lucia sehr zögerlich den Krümeln und [Departing entfernt] sich wieder [Source von ihnen], aber jetzt - jetzt hat sie einen beachtlichen Brocken erwischt, wendet sich sofort ab, und als der Trenchcoat des Mannes einen bedrohlichen Schwenk macht, fliegt sie auf in die Halle des Hauptbahnhofs. (Kopetzky, Steffen: Grand Tour, Frankfurt am Main: Eichborn 2002, S. 11)

Das Beispiel des lexikalischen Frames Departing zeigt, dass nicht nur Kern-FE, wie es bei Motion der Fall war, für die Motivierung der KtE der reflexiven Bewegungskonstruktion infrage kommen, sondern ebenso Nicht-Kern-FE, wie es bei Departing. $(\mathrm{PATH}\rangle$ der Fall ist.

Als letztes Beispiel möchte ich auf den lexikalischen Frame Bringing eingehen, der ebenfalls in der Benutzt-Relation in einer Frame-Nähe von +1 zu Motion steht und zugleich in der Siehe_auch-Relation, ebenfalls in einer Frame-Nähe von +1 . Die Siehe_auch-Relation konstituiert ,groups of frames that are similar and should be carefully differentiated, compared, and constrasted“ (Ruppenhofer et al. 2016: 85). Sie stellt eine schwache semantische Relation dar: „Due to its less structured semantic nature, the See_also relation freely violates any and all limitations normally expected for frame-to-frame relations“ (Ruppenhofer et al. 2016: 85).

Für den lexikalischen Frame Bringing und einige weitere, ähnlich gelagerte, Frames ergibt sich die Besonderheit, dass dass die KtE des KE BewEGENDES nicht, wie es zu in Analogie etwa zu Motion.THEME oder Departing.THEME zu erwarten sein könnte, durch das FE Bringing.THEME motiviert wird, sondern durch Bringing.AGENT. Dies ist etwa an den folgenden Belegen mit der LE schleppen (schlep.v) in (23) zu erkennen. 
(23) a. [AgENT Ich] [Bringing schleppe] mich [GoAL nach Hause], 2,5 km, schaffe es, die Tür aufzuschließen, die Treppe hoch, und da stehe ich auch schon in meinem Zimmer. (Braun, Marcus: Hochzeitsvorbereitungen, Berlin: Berlin Verlag 2003, S. 114)

b. [AGENT Schwere, sonnenlahme Schildkröten] [Bringing schleppten] sich [РАтн Wasser. (Düffel, John von: Houwelandt, Köln: DuMont Literatur und Kunst Verlag 2004, S. 142)

c. [AGENT Großvaters Uhrenzeiger] [Bringing schleppten] sich [SouRce von Minute] zu Minute, zauderten und zockelten, als seien sie widerwillige Pferde, und Zeigern konnte man mit einer Peitsche nicht kommen! (Koneffke, Jan: Paul Schatz im Uhrenkasten, Köln: DuMont Buchverlag 2000, S. 40)

d. Es waren verjährte Gedanken, kaum noch glimmende Hoffnungen, Gekränktsein, Klagen, ein Totengedenken war's: [AGENT Dutzende von Nekrologen] [Bringing schleppten] sich von Nummer [GoAL zu Nummer] fort und bildeten den finstersten und aufrichtigsten Teil des Blattes.“ (Schlögel, Karl: Petersburg, München Wien: Carl Hanser Verlag 2002, S. 606)

Diese Besonderheit, die auch auf weitere Frames wie etwa Cause_motion zutrifft, hängt mit der gleichzeitigen Motivierung des KEE zusammen, für die in diesem Beispiel das FE Bringing.THEmE vorbehalten ist. Ursache dafür ist die in den für das Englische entwickelten Frames wie Motion oder Departing nicht berücksichtigte Eigenheit des Deutschen, dass zahlreiche deutsche LE, die diese Frames evozieren, ,echt reflexive' Verben sind. Ich komme darauf in Unterabschnitt 6.3.1 im Zuge der Diskussion um die einfache Motivierung des KEE durch einen lexikalischen Frame zurück.

Welche Schlussfolgerungen lassen die Beobachtungen der semantischen Motivierung aller KtE der reflexiven Bewegungskonstruktion durch lexikalische Frames zu? Zunächst ist festzuhalten, dass in ihrer Analyse der way-Konstruktion schon Goldberg (1995: 207) zu dem Befund kommt, dass ,the verb may, but need not necessarily, code the semantics associated with the construction directly.“ Gleiches gilt hier auch für die reflexive Bewegungskonstruktion und Konstrukte, deren lexikalischer Frame Motion ist. Der Konstruktions-Frame kann sich somit direkt als von einigen LE, die das KE EREIGNIS instanziieren, evoziert, wiederfinden, wie dies ähnlich schon Goldberg (1999) für eine Reihe von Argumentstruktur-Konstruktionen gezeigt hat. Für die reflexive Bewegungskonstruktion (und auch die reflexive Partikelverbkonstruktion, aber nicht die reflexive Weg-Konstruktion) relevant ist zuvorderst die LE bewegen (move.v), die sich 
nicht zufällig im Namen der Konstruktion widerspiegelt (dazu Unterabschnitte 7.2.1 sowie 8.5.3).47 Die gebrauchsbasierte Perspektive legt zudem den Schluss nahe, dass der Konstruktions-Frame, wie aus der Argumentation von Goldberg (1999) ebenso abgeleitet werden kann, durch eine Generalisierung aller Instanzen, deren lexikalische Frames hier in einer Frame-Nähe zu Motion stehen, ermittelt werden kann (dazu Unterabschnitt 8.5.1). Gleiches gilt im Übrigen für die Benennung und Definition des KE EREIGNIS, das durch die LE lexikalischer Frames instanziiert wird (vgl. Unterabschnitt 7.3.1). Entscheidend für diese beiden Punkte ist eine hohe Frequenz dieser Konstrukte mit Motion als lexikalischem Frame sowie $\mathrm{zu}$ ihm relatierten lexikalischen Frames, die in einer Frame-Nähe zu Motion stehen. Wie Tabelle 6.2 zeigt, trifft diese Generalisierung tendenziell zu: Der lexikalische Frame Motion steht an dritter Stelle aller für die reflexive Bewegungskonstruktion belegten relatierten lexikalischen Frames, frequenter sind lediglich Body_movement und Self_motion.

Werden alle KtE durch einen lexikalischen Frame motiviert, hat ein zweiter Frame-Typ neben diesem lexikalischen Frame aus offensichtlichen Gründen keinen Anteil an der Konstitution eines Konstrukt-Frames, da keine FrameAnpassung stattfindet. ${ }^{48}$ Die Tatsache, dass für die Konstruktion derjenige lexikalische Frame belegt ist, der mit dem Konstruktions-Frame identisch ist, unterscheidet die reflexive Bewegungskonstruktion, aber auch die reflexive Partikelverbkonstruktion und die reflexive Weg-Konstruktion, von ihrem englischen Pendant. So lautet eine gängige, aber nicht empirisch verifizierte Annahme über die way-Konstruktion, dass ,[t]here is no verb which licenses a theta frame identical to that of the Way-construction. In fact, verbs which do denote directed motion inherently are not welcomed by the construction, [...].“ (Michaelis 2003a: 276-277).

Diese Beobachtung wirkt sich auf das Koerzionspotenzial der Konstruktion aus, das für die way-Konstruktion gerade aufgrund der angenommenen In-

47 Die Differenzierung zwischen lexikalischem Frame und lexikalischer Bedeutung (Unterabschnitt 4.1.1) ist wichtig, um festzuhalten, dass es sich keineswegs bei allen LE, die den lexikalischen Frame Motion evozieren, gleichermaßen um „'light’ verbs“ (Goldberg 1999: 202) handelt. Neben bewegen (move.v) kommt am ehesten noch begeben (go.v) dafür infrage. Verben wie mäandern (meander.v), schlängeln (snake.v) oder winden (wind.v), deren lexikalische Bedeutungen sich durch abweichende Standardwerte entsprechend davon unterscheiden, gehören - obwohl sie ebenfalls Motion evozieren - sicher nicht in diese Reihe (vgl. dazu die Diskussion um die Lesarten der Konstruktionen in Unterabschnitt 5.2.3). Boas (2008c: 31-43) stellt ähnliche Überlegungen zu Self_motion an. Vgl. zu dieser Problematik auch die Kritik von Dux (2020: 70, 111).

48 Boas (2008b: 23) sieht in solchen Fällen alle Verben mit einer lexikalischen Bedeutung, deren Frame alle KtE motivieren kann, als Mini-Konstruktionen an, sodass keine weitere abstrakte Konstruktion mehr benötigt wird. 
kompatibilität von Verben mit lexikalischen Bedeutungen, deren Frames dem Konstruktions-Frame entsprechen, als hoch gilt (vgl. Michaelis 2003a: 277). Belege wie diejenigen in (5)-(23) sind zwar als Konstrukte der reflexiven Bewegungskonstruktion einzustufen, aufgrund der Tatsache, ihre Konstrukt-Frames ausschließlich aus FE (relatierter) lexikalischer Frames bestehen, ist gerade bei dem lexikalischen Frame Motion keine klare Linie mehr zwischen lexikalischem Frame und Konstruktions-Frame zu finden (vgl. dazu ohne Bezug auf Frames Iwata 2008: 88, 99; Dewell 2011: 12, 17), ${ }^{49}$ da die Leistung des Konstruktions-Frames, einen lexikalischen Frame hin $\mathrm{zu}$ einem Konstrukt-Frame $\mathrm{zu}$ anzupassen (Unterabschnitt 4.4.1) und damit Koerzionseffekte auszulösen (Abschnitte 5.5 und 7.4), in diesem Fall nicht eintreten kann. Die Frage, welcher Frame nun im Sinne eines Koerzionseffekts ,gewinnt' (vgl. Michaelis 2003a: 268), lässt sich also nicht beantworten, da keine Koerzion vorliegt. Deutlich wird allerdings, dass der Konstruktions-Frame einen lexikalischen Frame nicht vollständig (d.h.: in der Motivierung aller KtE) anpassen kann: Ist der Konstruktions-Frame für die semantische Motivierung aller KtE verantwortlich, wie in den Belegen in (5)-(20), dann muss er mit dem lexikalischen Frame identisch sein. Eine Situation, in der alle KtE der Konstruktion ausschließlich durch die FE von Motion motiviert sind, der lexikalische Frame aber nicht diesem Frame entspricht, ist nicht möglich.

Die Identität von Konstruktions-Frame und lexikalischem Frame impliziert ganz im Sinne von Goldberg (1992: 48) eine Redundanz bei der Konstitution des Konstrukt-Frames und macht deutlich, warum gerade der lexikalische Frame und nicht der Konstruktions-Frame als für die Konstitution des Konstrukt-Frames wesentlich angesehen werden muss: Würde man annehmen, in den Belegen in (5)(20) sei ein separater Konstruktions-Frame am Werk, würde man LE wie begeben, bewegen, mäandern, schlängeln oder winden die Evokation eines lexikalischen Frames und damit eine lexikalische Bedeutung absprechen (vgl. für eine ähnliche Schlussfolgerung zur Ditransitivkonstruktion Croft 2001: 122).

Die Frage nach der Motivierung aller KtE einer Konstruktion durch einen lexikalischen Frame, der mit dem Konstruktions-Frame identisch ist oder in FrameNähe zu diesem steht, ist darüber hinaus, wie ich bereits in Unterabschnitt 5.4.2 angedeutet habe, als ein wichtiges Indiz dafür anzusetzen, ob gegebene Belege tatsächlich Konstrukte einer zu untersuchenden Konstruktion zeigen (dazu Unterabschnitt 8.4.2). Alle Konstrukte, deren KtE durch einen relatierten lexikalischen Frame motiviert werden, sind als Instanzen dieser Konstruktion zu werten. Aus-

\footnotetext{
49 Mit anderen Worten: „[T]here is no reason why the verb meaning and the constructional meaning should not overlap.“ (Iwata 2008: 88). Ebenso schreibt auch Langacker (2009a: 248): „Lexical and constructional meaning overlap and are often non-distinguishable.“
} 
schlaggebend ist die Tatsache, dass alle KtE vollständig durch einen solchen und keinen anderen - lexikalischen Frame motiviert werden können. Für den Fall lexikalischer Frames, die nicht direkt dem Konstruktions-Frame entsprechen, zu ihm aber in einer Frame-Nähe stehen, lässt sich mit Perek (2015: 28) von einer „inherent compatibility“ zwischen lexikalischem Frame und Konstruktions-Frame sprechen. Hierbei ist es der Fall, dass ,both the verb and the construction map onto the same elements of form and meaning in the clause: they contribute the same number and types of arguments“ (Perek 2015: 28).

Diese Befunde sind Evidenz für die in Abschnitt 4.2 erwähnte Vorrangstellung lexikalischer Frames. ${ }^{50}$ Selbst wenn Konstruktions-Frame und lexikalischer Frame nicht identisch sind, ist es möglich, dass der lexikalische Frame alle KtE eines Konstrukts motiviert. Dabei kann er spezifischer als der Konstruktions-Frame sein (vgl. ähnlich Perek 2015: 29-30), solange es sich um einen relatierten lexikalischen Frame handelt. Für lexikalische Frames, die in Vererbungsrelation mit einer Frame-Nähe von +1 oder niedriger zum Konstruktions-Frame stehen (wie etwa Self_motion), ist dies trivial: Sie sind bereits durch die Natur dieser Relation spezifischer als der Konstruktions-Frame. Allerdings muss ein relatierter lexikalischer Frame nicht zwingend spezifischer als der Konstruktions-Frame sein: Manche Frame-zu-Frame-Relationen, wie die Kausativ-Relation (mit Cause_motion), können lexikalische Frames bereitstellen, die mit einer Frame-Nähe wie -1 abstrakter als der Konstruktions-Frame sind.

Obwohl, wie oben ausgeführt, aufgrund einer fehlenden Frame-Anpassung kein unmittelbarer Einfluss eines separaten Konstruktions-Frames bei relatierten lexikalischen Frames (mit einer Frame-Nähe von +1 oder niedriger bzw. -1 oder niedriger) gegeben ist, lässt sich nicht behaupten, dass der Konstruktions-Frame bei lexikalischen Frames, die in einer Frame-Nähe zu ihm stehen, keine Rolle in der einfachen Motivierung von Konstrukten spielen würde. Dadurch, dass diese lexikalischen Frames zum Konstruktions-Frame relatiert sind, ist eine ,indirekte ${ }^{6}$ Verbindung zu Letzterem stets gegeben. Tabelle 6.2 zeigt, dass diese lexikalischen Frames, die eben nicht mit dem Konstruktions-Frame identisch sind, aber zu ihm in Frame-Nähe stehen, quantitativ sogar in der Mehrheit sind. Deren (je nach Frame-Nähe mehr oder weniger), indirekte‘ Verbindung zum KonstruktionsFrame ist ein wesentliches Argument dafür, auch bei Konstrukten, deren KtE vollständig durch einen lexikalischen Frame einfach motiviert werden, welcher nicht dem Konstruktions-Frame entspricht, von Instanzen der Konstruktion zu spre-

50 Mit Verweis auf Michaelis \& Ruppenhofer (2001: 49-50) stellt auch Diedrichsen (2014: 185) fest, ,dass sowohl verbale als auch konstruktionsbasierte Semantik in die Bedeutung einer Argumentstruktur-Konstruktion [richtiger wäre: eines Konstrukts, A.W.] einfließen können, wobei die Konstruktionsanforderungen der Verbsemantik vorrangig sind“. 
chen. Zudem ist diese ,indirekte' Verbindung zum Konstruktions-Frame ein wesentlicher Motor für die Evokation des Konstruktions-Frames bei relatierten lexikalischen Frames (vgl. Unterabschnitt 8.2.2).

Dass das Kriterium der ,indirekten' Verbindung zum Konstruktions-Frame entscheidend ist, lässt sich an Instanzen illustrieren, die zwar eine strukturelle Analogie zur reflexiven Bewegungskonstruktion aufweisen, die allerdings gerade nicht als Instanzen dieser Konstruktion gelten können. Dies trifft auf Konstrukte $\mathrm{zu}$, die durch einen lexikalischen Frame vollständig einfach motiviert werden, der in keiner Frame-Nähe zu Motion steht, also nicht relatiert ist. ${ }^{51}$ Die folgenden Belege (24)-(34) sind nur einige Beispiele für solche Fälle. ${ }^{52}$ Zur Übersicht sind nur die jeweiligen LE auf die entsprechenden lexikalischen Frames annotiert.

(24) Die französische Autorin [contacting wendet] sich mit ihren elf Reportagen zunächst an die Franzosen, bei denen die Deutschen unbeliebt sind. (Die Zeit, 20.01.2000, Nr. 4)

(25) Carl [Memory erinnert] sich genau an den Vormittag, als wir herausfanden, daß ihr Vater das Haus offenbar bereits vor einiger Zeit verlassen hatte, die Zahnbürste stand noch im Becher, das Rasierzeug war noch da, aber die Reisetasche fort. (Beyer, Marcel: Spione, Köln: DuMont 2000, S. 235)

(26) Der Preis [purpose richtet] sich gleichermaßen an Printjournalisten wie an die Kollegen in den elektronischen Medien. (Die Zeit, 24.02.2000, Nr. 9)

(27) Die Tester [Limiting beschränken] sich auf Adressen, wichtige Fakten und jede Menge Symbole. (Die Zeit, 27.01.2000, Nr. 5)

(28) 128 Unterzeichnerstaaten [Make_agreement_on_action einigten] sich am frühen Morgen des vergangenen Samstags in Montreal auf gemeinsame Regeln für den Handel mit gentechnisch veränderten Organismen. (Die Zeit, 03.02.2000, Nr. 6)

(29) Sie, wie auch ihre Kollegen von Audi und BMW, [Rel iance verlassen] sich bei der Abwehr des französischen Ansinnens ganz auf Gerhard Schröder, den „Kanzler aller Autos“. (Die Zeit, 20.01.2000, Nr. 4)

51 Auf eine Ausnahme, die für die reflexive Partikelverbkonstruktion belegt ist, komme ich in Unterabschnitt 6.4.3 zu sprechen.

52 Zur Herkunft der Daten vgl. Unterabschnitt 3.4.2. Die hier einschlägigen englischen LE sind (in der Reihenfolge der Belege) contact.v, remember.v, aim.v, limit.v, agree.v, rely.v, prevail.v, defend.v, oppose.v, love.v und transform.v. 
(30) Am 16. Januar [Beat_opponent setzte] sich die bisherige Außenministerin Tarja Halonen gegen ihren schärfsten Konkurrenten Esko Aho durch. (Archiv der Gegenwart, 2001 [2000])

(31) Auch Achims Freunde [Defending wehren] sich gegen Puder und Cremes. (Die Zeit, 13.01.2000, Nr. 3)

(32) Er [Taking_sides wandte] sich gegen die Meinung, Verbrechen sei eine Folge sozialer Unterdrückung, Straftäter seien also Opfer gesellschaftlicher Zustände. (Die Zeit, 13.01.2000, Nr. 3)

(33) Das Mädchen mit den weißen Haaren [Experiencer_focus verliebt] sich in den Mann mit dem Sportwagen. (Die Zeit, 13.01.2000, Nr. 3)

(34) Diese Furcht [undergo_transformation verwandelt] sich mehr und mehr in ein Vorurteil, um nicht zu sagen, in eine Ausrede. (Die Zeit, 05.01.2000, Nr. 2)

Solche Falschpositive, die bei der Analyse der reflexiven Bewegungskonstruktion ausgeschlossen werden müssen, sind eindeutig nicht als Instanzen der reflexiven Bewegungskonstruktion einzustufen, weil sie durch lexikalische Frames einfach motiviert werden, die in keiner Frame-Nähe zu Motion stehen. Dieser ist als Konstruktions-Frame damit weder durch eine Frame-Anpassung und einen Blend mit einem lexikalischen Frame an der Konstitution der Konstrukt-Frames beteiligt (vgl. dazu die Unterabschnitte 6.2.3 und 6.3.2), noch ,im Hintergrund' dadurch, dass ihre lexikalischen Frames in einer Frame-Nähe zum angenommenen Konstruktions-Frame stehen. ${ }^{53}$

Nebenbei sind solche Falschpositive ein gewichtiges Argument dafür, dass Konstruktionen nicht über rein formale Strukturen definiert werden können, sondern dass dies durch semantische Benennungen und Definitionen ihrer Strukturelemente geschehen muss (vgl. Unterabschnitt 6.1.2 sowie Abschnitt 7.3). Würde man für die reflexive Bewegungskonstruktion lediglich eine formale Definition ansetzen, würden Belege wie (24)-(34) als Konstrukte dieser Konstruktion zählen, was unplausibel ist. Die Tatsache, dass allen diesen Belegen lexikalische Frames zugrundeliegen, die in keiner Verbindung zum angenommenen KonstruktionsFrame stehen, ist ein empirischer Nachweis dieser Unplausibilität. Einer Antwort

53 Netzwerktheoretisch lässt sich hier Anschluss an Spreading-Activation-Modelle finden (vgl. überblickend Barsalou 1992a: 45-49; Traugott \& Trousdale 2013: 54-56; Diessel 2019: 94; Schmid 2020: 44-45): Wird ein lexikalischer Frame, der in Frame-Nähe zum Konstruktions-Frame steht (z.B. Body_movement) in einem Konstrukt evoziert, wird der Konstruktions-Frame (Motion) zugleich mitaktiviert, da er in einer Frame-zu-Frame-Relation zu dem betreffenden lexikalischen Frame steht, auch wenn der Konstruktions-Frame selbst nicht im Konstrukt evoziert wird. Ich komme darauf in Unterabschnitt 8.2.2 für die Evokation des Konstruktions-Frames zurück. 
auf die Frage danach, ob ein vorliegender Beleg als Instanz einer gegebenen Konstruktion einzustufen ist, lässt sich durch den Test, ob dessen lexikalischer Frame in einer Frame-Nähe zum angenommenen Konstruktions-Frame steht, näherkommen. Diese Befunde bilden den ersten Schritt zur Entwicklung einer Methode, den Konstruktions-Frame zu ermitteln - eine für eine Konstruktionssemantik und die Konstruktikographie zentrale Aufgabe. Ich komme darauf in Unterabschnitt 8.4.2 zurück.

\subsubsection{Einfache Motivierung einzelner KtE durch Konstruktions-Frame}

Der erste zur einfachen Motivierung aller KtE eines Konstrukts durch FE des lexikalischen Frames entgegengesetzte Fall liegt vor, wenn ein KtE durch ein einzelnes FE des Konstruktions-Frames motiviert ist - und zwar ausschließlich durch ein solches -, während andere KtE noch durch FE eines unrelatierten lexikalischen Frames motiviert werden. Es handelt sich dabei um die in Unterabschnitt 4.4.1 erläuterte Variante a einer Frame-Anpassung. Der Anteil des KonstruktionsFrames bei der Konstitution des Konstrukt-Frames ist dann insofern als stark zu bewerten, als dass der lexikalische Frame in der semantischen Motivierung des betreffenden KtE keine Rolle mehr spielt und die Motivierung durch ein FE des Konstruktions-Frames übernommen wird (dazu auch Unterabschnitt 7.4.1). Mit anderen Worten: Das KtE wird einzig durch den Konstruktions-Frame lizenziert und das FE, das es motiviert, ist nicht im lexikalischen Frame enthalten. ${ }^{54}$ Aus der Perspektive des lexikalischen Frames wäre die Konstruktion in diesen Fällen „described as augmenting the valence of an independently-licensed sign." (Fillmore, Lee-Goldman \& Rhomieux 2012: 325) und die konzeptuelle Integration von lexikalischem Frame und Konstruktions-Frame „results in augmentation of the verbal valence.“ (Michaelis 2005: 57). ${ }^{55}$ Aus dieser Perspektive besteht die Leistung des Konstruktions-Frames in seiner „capability of contributing arguments to the se-

54 Deshalb gilt die von Jackendoff (2002: 174) für die way-Konstruktion getroffene Annahme, die PP (also das KtE des KE WEG) sei (immer?) ein semantisches Argument der Konstruktion, nur für unrelatierte lexikalische Frames. In relatierten lexikalischen Frames hingegen ist das KtE des KE WEG immer ein Argument des KtE EREIGNIS, also des Verbs und damit der LE, die den lexikalischen Frame evoziert.

55 Dementsprechend ordnen etwa Sag (2012: 140-145) und Hilpert (2019: 36-38) die wayKonstruktion in die Kategorie valenzerhöhender Konstruktionen ein. Für Welke (2019: 200) handelt es sich bei den solche Konstruktionen auszeichnenden zusätzlichen KtE um „[ü]berschüssige Argumente“, die er als Koerzionseffekte unter dem Begriff der Valenzerhöhung (vgl. Welke 2019: 239-243) oder Valenzerweiterung (vgl. Welke 2011: 199-249) diskutiert. Vgl. auch die Argumentation von Michaelis \& Ruppenhofer (2001: 9) für Konstruktionen, die Valenz nicht nur erwei- 
mantics of a verb.“ (Boas 2008a: 128). Formal gesehen wird die Argumentstruktur als Ganze in einem solchen Fall von den Eigenschaften der LE und der Konstruktion gemeinsam determiniert (vgl. Goldberg \& Jackendoff 2004: 534). Hinsichtlich der Frame-Anpassung des lexikalischen Frames wird dieser im Konstrukt-Frame um ein FE des Konstruktions-Frames erweitert.

Im Sinne der Theorie der konzeptuellen Integration besteht im Falle einer einfachen Motivierung durch den Konstruktions-Frame keine Gegenstück-Relation zwischen dem FE des Konstruktions-Frame und einem FE des lexikalischen Frames, da Letzterer eben kein FE enthält, das die Motivierung des KtE übernehmen könnte. Es handelt sich dabei um eine einfache Projektion aus dem Konstruktions-Frame in den Konstrukt-Frame (vgl. Unterabschnitt 4.4.2). Die einfache Motivierung eines KtE durch den Konstruktions-Frame ist das deutlichste Anzeichen einer nicht vorhandenen Frame-Nähe eines lexikalischen Frames zum Konstruktions-Frame. Eine vorhandene Frame-Nähe ist also kein notwendiges Kriterium dafür, dass eine Instanz als Konstrukt einer gegebenen Konstruktion gelten kann:

While the meanings of collexemes are usually compatible with the meaning of the construction, this is not always the case. As we have seen, the co-occurrence of verbs and argumentstructure constructions exhibits a great deal of semantic idiosyncrasy. (Diessel 2019: 123)

Mehr noch: Steht der lexikalische Frame nicht in Frame-Nähe zum KonstruktionsFrame, lässt dies ein Konstrukt durch die Frame-Anpassung des lexikalischen Frames durch den Konstruktions-Frames bei der Konstitution des Konstrukt-Frames besonders ,interessant‘ werden:

More interestingly [...] are cases wherein the verb does not itself lexically designate the meaning associated with the construction, in which case we have two distinguishable events. (Goldberg 2010: 53)

In einem Fall wie diesem, in dem der Konstruktions-Frame den lexikalischen Frame um ein FE anpasst, das darin nicht angelegt ist, liegt ein Koerzionseffekt vor (vgl. auch Welke 2019: 29), der sich in eine von sieben Koerzionsstufen (Unterabschnitt 7.4.1) einordnen lässt. Innerhalb der Klassifikation von Koerzionsphäno-

tern, sondern auch (im Falle nicht-relationaler Wortarten) kreieren können. Die reflexive Bewegungskonstruktion (und auch die reflexive Partikelverbkonstruktion und die reflexive WegKonstruktion) als Ganzes aber als eine valenzerweiternde Konstruktion zu charakterisieren, halte ich für problematisch, da die Valenzerweiterung lediglich eine Teilmenge lexikalischer Frames (nämlich nur unrelatierte) und damit eine Teilmenge von LE, die das KE EREIGNIS instanziieren können, betrifft. 
menen von Audring \& Booij (2016: 629-631) entspricht die Anpassung des lexikalischen Frames um ein FE des Konstruktions-Frames einer coercion by override (vgl. ähnlich auch Perek 2015: 28).

Zur Konstitution des Konstrukt-Frames ist der lexikalische Frame demnach nicht ausreichend, er muss durch den Konstruktions-Frame erweitert werden. Für die vorliegenden Fälle heißt das konkret, dass die semantische Motivierung eines KtE, das nicht durch ein FE des lexikalischen Frames motiviert werden kann, durch den Konstruktions-Frame ,beigetragen werden muss. ${ }^{56}$ Auf einer Skala unterschiedlicher Koerzionsstufen (Unterabschnitt 7.4.1) tendieren Konstrukte wie die, die ich im Folgenden diskutieren möchte, zu deutlichen Koerzionseffekten.

Für die reflexive Bewegungskonstruktion, aber auch die anderen beiden untersuchten Konstruktionen, betrifft eine solche einfache Motivierung eines einzelnen KtE durch den Konstruktions-Frame in erster Linie das KE WEG bzw. 〈WEG). ${ }^{57}$ Seine KtE werden durch den Konstruktions-Frame insbesondere dann einfach motiviert, wenn der lexikalische Frame kein zu den FE Motion.SourCE Motion.PATH, Motion.GoAl oder Motion.DiRection äquivalentes FE enthält, das das KtE motivieren könnte. ${ }^{58}$ Insgesamt 91 Konstrukte der reflexiven Bewegungskonstruktion

56 Szcześniak (2014a: 145) reduziert die way-Konstruktion gar auf diese Eigenschaft, ein KtE wie WEG der lexikalischen Bedeutung hinzuzufügen - eine Eigenschaft, die sogar seiner ansonsten vorgebrachten Kritik an deren ,Bedeutungshaltigkeit“ (vgl. auch Unterabschnitte 4.3.3 und 5.7.2) standhält.

57 Vgl. bereits Levin \& Rapoport (1988: 278), die für die way-Konstruktion darauf hinweisen, dass durch die Präposition (hier also den Kopf der KtE des KE WEg bzw. 〈WEG〉) ein vom Verb unabhängiger semantischer Beitrag geleistet wird. Rohde (2001: 116-128) untersucht für das Englische systematische Kookkurrenzen zwischen ,Bewegungsverben` und ,Nicht-Bewegungsverben` und einzelnen Typen von Präpositionen. Auf die besondere Rolle von PP (als die die KtE des KE WEG realisiert werden) bei der Einführung eines neuen mental space in ein Netzwerk der konzeptuellen Integration weist Fauconnier (1997: 40) hin.

58 Einer der Gründe, warum im Deutschen (wie im Englischen) ein durch ein FE wie Motion.SourCE, Motion.PATH, Motion.GoAl oder Motion.DiRECTION motiviertes KtE überhaupt mittels einer eigenständigen Konstituente ausgedrückt werden kann, liegt in dem Charakteristikum unter anderem der indoeuropäischen Sprachfamilie (außer den romanischen Sprachen), diese semantischen Aspekte einer Bewegungsbedeutung in vom Verbstamm getrennte,Satelliten auszulagern, anstatt sie direkt im Verb zu kodieren (vgl. Talmy 2007: 138-163). Diese Beobachtung dürfte nicht nur die Herkunft der formalen Struktur der Konstruktion als solche erklären, sondern gleichzeitig ihre Produktivität begründen, denn die produktive Erweiterung eines Verbs um unterschiedlichste Arten von ,Satelliten` erscheint einfacher möglich als die Etablierung ,neuer Verbstämme - gerade, wenn das im ,Satelliten` instanziierte FE nicht in der lexikalischen Bedeutung des Verbs angelegt ist, sondern, wie hier, dem Konstruktions-Frame entstammt. Vor diesem Hintergrund wäre eine typologische Studie wünschenswert, die nach der Existenz einer der reflexiven Bewegungskonstruktion bzw. der way-Konstruktion äquivalenten Konstruktion in Sprachen, die semantische Aspekte wie ,РATH“ direkt am Verb kodieren, fragt. 
sind davon betroffen. Tabelle 6.3 zeigt deren Verteilung nach lexikalischen Frames.

Tab. 6.3: Einfache Motivierung von KtE des KE WEG der reflexiven Bewegungskonstruktion durch den Konstruktions-Frame nach lexikalischen Frames

\begin{tabular}{lrr}
\hline Lexikalischer Frame & Konstrukte & Anteil \\
\hline Daring & 18 & $19,78 \%$ \\
Manipulation & 16 & $17,58 \%$ \\
Cause_to_experience & 11 & $12,09 \%$ \\
Hostile_encounter & 11 & $12,09 \%$ \\
Rescuing & 5 & $5,49 \%$ \\
Work & 4 & $4,40 \%$ \\
Chatting & 3 & $3,30 \%$ \\
Make_noise & 3 & $3,30 \%$ \\
Reshaping & 3 & $3,30 \%$ \\
Seeking & 3 & $3,30 \%$ \\
Cause_to_move_in_place & 2 & $2,20 \%$ \\
Ingestion & 2 & $2,20 \%$ \\
Progression & 2 & $2,20 \%$ \\
Attaching & 1 & $1,10 \%$ \\
Cause_bodily_experience & 1 & $1,10 \%$ \\
Cause_harm & 1 & $1,10 \%$ \\
Dead_or_alive & 1 & $1,10 \%$ \\
Hunting & 1 & $1,10 \%$ \\
Impact & 1 & $1,10 \%$ \\
Prevarication & 1 & $1,10 \%$ \\
Shopping & 1 & $1,10 \%$ \\
\hline Gesamt & 91 & $100,00 \%$ \\
\hline
\end{tabular}

Der frequenteste lexikalische Frame, der im Konstrukt-Frame um ein FE des Konstruktions-Frame ergänzt wird, ist Daring. Die innerhalb dieses lexikalischen Frames wiederum frequentesten LE sind (ge)trauen und wagen (beide dare.v). Die Belege in (35) und (36) sind Beispiele für die Motivierung von KtE des KE WEG durch das FE Motion.GoAL des Konstruktions-Frames, das, wie ich gleich zeigen werde, das frequenteste FE des Konstruktions-Frame in dieser Art der Motivierung der KtE des KE WEG ist. ${ }^{59}$

59 Aus Gründen der besseren Lesbarkeit ist bei allen Beispielen, in denen lediglich ein KtE von Interesse ist, einzig dessen frame-semantische Annotation dargestellt. Die Annotation der anderen KtE wird hierfür ausgeblendet. Zur Erinnerung: Die Annotation der FE des Konstrukt-Frames 
(35) a. Ich [Daring traute] mich kaum [an sie $\left.{ }^{\text {GoAL}}\right]$ heran. (Goosen, Frank: Liegen lernen, Frankfurt am Main: Eichborn AG 2000, S. 295)

b. Simons Tochter [Daring wagt] sich unter Lebensgefahr [zu ihrem Vater ins Verlies GoAL] und rettet ihn, indem sie ihn mit ihrer Brust nährt, vor dem Verhungern. (Wondratschek, Wolf: Mozarts Friseur, München, Wien: Carl Hanser Verlag 2002, S. 110)

c. Er machte sich Vorwürfe und [Daring getraute] sich aus schlechtem Gewissen nicht einmal mehr [zu seinen Kindern GoAL]. (Wondratschek, Wolf: Mozarts Friseur, München, Wien: Carl Hanser Verlag 2002, S. 79)

(36) a. Trotzdem [Daring wagen] sich laufend neue B2B-Firmen [an die Börse GoAL]. (Die Zeit, 03.02.2000, Nr. 6)

b. Er meidet diese Gegend, [Daring wagt] sich nicht [auf den Hügel GoAL] herauf, fürchtet noch den entferntesten Blickkontakt mit einem Mitglied seiner Familie. (Beyer, Marcel: Spione, Köln: DuMont 2000, S. 126)

c. Seit es nicht mehr allzu riskant ist, [Daring wagen] sich auch andere aus der jüngeren CDU-Generation [gegen den Altkanzler GoAL] vor. (Die Zeit, 03.02.2000, Nr. 6)

d. Weil die Probleme offenbar noch nicht reichen, [Daring wagt] sich Volkswagen auch noch [in die Luxusregionen GoAL]. (Die Zeit, 30.03. 2000, Nr. 14)

e. Das Kleeblatt [Daring wagt] sich [nach draußen ${ }^{\mathrm{GoAL}}$ ]. (Braun, Marcus: Hochzeitsvorbereitungen, Berlin: Berlin Verlag 2003, S. 19)

Neben Motion.GoAL kann der lexikalische Frame Dar ing im Konstrukt-Frame aber ebenso durch Motion.SouRcE erweitert werden. Die Belege in (37) enthalten die einzigen beiden Konstrukte dafür mit der LE trauen (dare.v).

a. Kopp [Daring traute] sich nicht mehr [aus seinem Zimmer SourcE]. (Koneffke, Jan: Paul Schatz im Uhrenkasten, Köln: DuMont Buchverlag 2000, S. 74)

b. Er [Daring traute] sich nicht mehr [aus dem Haus Source], hörte immer wieder Stimmen, fühlte sich bedroht und sah im Spiegel jemanden, den er nicht kannte. (Die Zeit, 09.03.2000, Nr. 11)

Der lexikalische Frame Manipulation ist, wie Tabelle 6.3 zeigt, nahezu gleichfrequent mit Daring. Auch er wird in den Konstrukt-Frames, an denen er beteiligt

ist hinter dem jeweiligen KtE hochgestellt angegeben. Da der Konstruktions-Frame stets Motion ist und diese FE ihm entstammen, ist sein Name nicht separat in der Annotation vermerkt. 
ist, in der Mehrheit der Konstrukte um das FE Motion.GoAL ergänzt, das das KtE des KE WEG einfach motiviert. Entsprechende Belege für die LE zwängen und quetschen (beide squeeze.v) sind unter (38) und (39) zusammengestellt. ${ }^{60}$

a. Plötzlich verschwand die Robbe von unserer Seite und [Manipulation zwängte] sich [in die Menschenkette vor den Uniformierten GoAL]. (Schulze, Ingo: Neue Leben, Berlin: Berlin Verlag 2005, S. 485)

b. Sie legte das Klassenbuch auf den Lehrertisch und [Manipulation zwängte] sich, ohne ihren Stuhl zurückzuschieben, [auf ihren Platz ${ }^{\text {GoAL}}$ ]. (Schulze, Ingo: Neue Leben, Berlin: Berlin Verlag 2005, S. 759)

(39) a. Sie [Manipulation quetschten] sich ebenfalls [in den Raum, der nicht viel größer als das Ehebett war, das in ihm stand ${ }^{\text {GoAL }}$. (Widmer, Urs: Das Buch des Vaters, Zürich: Diogenes 2004, S. 142)

b. Die Babylonier nickten und [Manipulation quetschten] sich ungelenk durch die Glastür [auf die Steinstraße GoAL]. (Kopetzky, Steffen: Grand Tour, Frankfurt am Main: Eichborn 2002, S. 283)

c. „Ja, bitte, kommen Sie mit“, rief der Dicke und [Manipulation quetschte] sich [nach hinten GOAL], während ich vorn Platz nehmen durfte. (Schulze, Ingo: Neue Leben, Berlin: Berlin Verlag 2005, S. 544)

Neben der Ergänzung des lexikalischen Frames Manipulation durch das FE Motion.GoAL in einem Konstrukt-Frame ist ebenso eine Motivierung entsprechender KtE des KE WEG durch das FE Motion.PATH belegt. Die Belege (40) und (41) sind Beispiele hierfür. Hinsichtlich der LE, die als KtE des KE EREIGNIS instanziieren, dominieren auch hier quetschen und zwängen (beide squeeze.v).

60 Im Deutschen scheinen die Verben zwängen und quetschen allerdings allzu regelmäßig mit direktionalen PP aufzutreten, wie es etwa die entsprechenden Einträge im DWDS-Wörterbuch suggerieren, in denen sich Beispiele wie seine Hand durch ein Gitter zwängen (https://www.dwds. de/wb/zwängen, zuletzt abgerufen am 07.09.2021) oder jmd. an die Wand, gegen eine Mauer quetschen (https://www.dwds.de/wb/quetschen, zuletzt abgerufen am 07.09.2021) finden. Beide Einträge enthalten zudem Beispiele, die als Konstrukte der reflexiven Bewegungskonstruktion einzuordnen wären (z.B. sie zwängte sich in einen überfüllten Bus; sie quetschten sich in das kleine Auto, volle Zugabteil, durch die enge Tür). Der lexikalische Frame Manipulation jedoch verfügt über kein FE, das entsprechende PP motivieren könnte, weshalb hier der Konstruktions-Frame Motion hinzukommen muss. Es bleibt freilich möglich, dass es sich hier um ein Charakteristikum des für das Englische entwickelten Frames handelt, das für das Deutsche nicht gilt. Für eine Anpassung des Frames Manipulation an das Deutsche wäre also ggf. ein entsprechendes FE zu erwägen, was dazu führen würde, dass in Konstrukten wie denen in (38)-(41) die KtE des KE WEG durch den lexikalischen Frame motiviert würden. 
(40) Die Babylonier nickten und [Manipulation quetschten] sich ungelenk [durch die Glastür ${ }^{\mathrm{PATH}}$ ] auf die Steinstraße. (Kopetzky, Steffen: Grand Tour, Frankfurt am Main: Eichborn 2002, S. 283)

(41) a. Der Ministerpräsident [Manipulation zwängt] sich samt Tross [durch die kahlen Betonkavernen $\left.{ }^{\mathrm{PATH}}\right]$, sieht die armen Betten dicht an dicht, dazu die Notmatratze. (Die Zeit, 13.04.2000, Nr. 16)

b. Auf dem Absatz machte ich kehrt und [Manipulation zwängte] mich wieder zwischen Stoßstangen und Auspuffrohren [über die Straße ${ }^{\text {РАтн] }}$. (Franck, Julia: Lagerfeuer, Köln: DuMont Literatur und Kunst Verlag 2003, S. 257)

c. Auf dem Absatz machte ich kehrt und [Manipulation zwängte] mich wie-

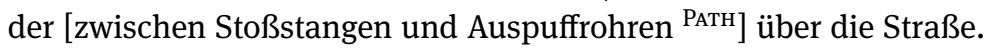
(Franck, Julia: Lagerfeuer, Köln: DuMont Literatur und Kunst Verlag 2003, S. 257)

Die lexikalischen Frames Cause_to_experience und Hostile_encounter, die hinsichtlich der Ergänzung um ein FE des Konstruktions-Frames in den KonstruktFrames, in denen sie beteiligt sind, dieselbe Frequenz aufweisen, sind ebenso wie die beiden soeben diskutierten Frames mit unterschiedlichen FE des Konstruktions-Frames belegt. Sowohl für Cause_to_experience als auch für Hostile_encounter dominiert das FE Motion.PATH, vereinzelt sind aber auch Konstrukt-Frames mit Motion.GoAL belegt. Die Belege in (42) sind Beispiele für Cause_to_experience und das FE Motion.PATH, während Beleg (43) ein Beispiel für denselben Frame und das FE Motion.GoAL zusammengestellt ist.

(42) a. Eine Ahnung von Licht [Cause_to_experience quält] sich [durch ein briefbogengroßes, verdrecktes Fenster ${ }^{\mathrm{PATH}}$ ]. (Die Zeit, 24.02.2000, Nr. 9)

b. Vom sonnigen unteren Centovalli aus steigt man über fette Feuersalamander durch Esskastanienwälder bergan, schwitzt, dampft, springt nackt in einen Bergbach, [cause_to_experience quält] sich [über verrutschte Pfade ${ }^{\mathrm{PATH}}$ ] an Ziegenvolk vorbei ins Baumlose, zieht einen Pullover an, wirft den ersten Schneeball, und bei 1000 Metern über null versinkt man bis zur Hüfte im Schnee. (Die Zeit, 30.03.2000, Nr. 14)

(43) Lukian [Cause_to_experience quält] sich [zu einem Lächeln GoAL]. (Krausser, Helmut: Eros, Köln: DuMont 2006, S. 146)

Für den lexikalischen Frame Hostile_encounter ist (44) ein Beispiel für das FE Motion.PATH, während (45) einen Beleg für das FE Motion.GoAL zeigt. 
(44) David [Hostile_encounter kämpfte] sich [durch das überfüllte Lokal ${ }^{\text {PATH}}{ }^{\text {] }}$ und reduzierte seinen Wortschatz auf: Moment, gleich, Sekunde, bin schon da und sofort. (Suter, Martin: Lila, Lila, Zürich: Diogenes 2004, S. 52)

(45) Sie haben die Menschenrechte zur Richtschnur der Außenpolitik erklärt und [Hostile_encounter ringen] sich [zu keiner Verurteilung des TschetschenienKrieges ${ }^{\text {GoAL }}$ ] durch. (Die Zeit, 23.03.2000, Nr. 13)

Für Hostile_encounter ist zudem ein Konstrukt-Frame mit Motion.DIRECTION belegt, vgl. (46).

(46) Das von Uganda unterstützte MLC BEMBAs, mittlerweile eine der größten Rebellengruppen, kontrolliert weite Gebiete im Osten und Norden des Kongo und [Hostile_encounter kämpft] sich [in Richtung der Hauptstadt Kinshasa Direction] vor. (Archiv der Gegenwart, 2001 [2000])

Es wird also deutlich, dass es sich bei dem FE, das aus dem Konstruktions-Frame in den Konstrukt-Frame eingeht und so den lexikalischen-Frame anpasst - auch innerhalb ein und desselben lexikalischen Frames -, nicht stets um dasselbe FE handeln muss. In Tabelle 6.4 ist die Verteilung für die vier FE Motion.SourcE, Motion.PATH, Motion.GoAL und Motion.DiRECTION dargestellt. Wie leicht zu erkennen und auch erwartbar ist, hängt die Wahl des FE des Konstruktions-Frames stark von der Präposition ab, die den Kopf des KtE des KE WEG bildet.

Während die Frage, welches FE des Konstruktions-Frames ein einzelnes KtE motiviert, bis zu einem gewissen Grad mit Blick auf die Präposition, die den Kopf des KtE des KE WEG bildet, beantwortet werden kann, existiert ein weiterer Einflussfaktor, von dem die Motivierung des KtE abhängig ist: die lexikalische Bedeutung. Die lexikalischen Bedeutungen, die die unterschiedlichen LE eines Frames evozieren, können die Motivierung des KtE des KE WEG dahingehend differenzieren, ob das KtE überhaupt durch den Konstruktions-Frame motiviert wird oder nicht bereits über den lexikalischen Frame. Ein Beispiel hierfür ist der lexikalische Frame Cause_to_move_in_place. Für die lexikalischen Bedeutungen von LE wie wiegen (rock.v) und wellen (wave.v) erscheint es plausibel, dass das KtE des KE WEG durch ein FE wie FE Motion.GoAL (47) oder Motion.PATH (48) motiviert wird.

(47) Sie [Cause_to_move_in_place wiegte] sich [gegen ihn GoAL]. (Hettche, Thomas: Der Fall Arbogast, Köln: DuMont Buchverlag, 2001, S. 14)

(48) Als sie den Finger auf eine blaue Blüte schob, färbte sich ihr Fingernagel bläulich und grüne Streifen [Cause_to_move_in_place wellten] sich [über ihren Handrücken ${ }^{\mathrm{PATH}}$ ]. (Pressler, Mirjam: Malka Mai, Weinheim Basel: Beltz \& Gelberg 2001, S. 5) 
Tab. 6.4: FE des Konstruktions-Frames Motion in einfacher Motivierung der KtE des KE WEG der reflexiven Bewegungskonstruktion nach Präpositionen

\begin{tabular}{lrrrr}
\hline Präposition & Motion.GoAL & Motion.PATH & Motion.SOURCE & Motion.DiReCtion \\
\hline$a b$ & 0 & 0 & 0 & 0 \\
an & 6 & 0 & 0 & 0 \\
auf & 7 & 0 & 0 & 0 \\
aus & 0 & 0 & 6 & 0 \\
bis & 1 & 0 & 0 & 0 \\
durch & 0 & 27 & 0 & 0 \\
gegen & 2 & 0 & 0 & 0 \\
hinter & 0 & 0 & 0 & 0 \\
in & 19 & 0 & 0 & 2 \\
nach & 2 & 0 & 0 & 0 \\
neben & 0 & 0 & 0 & 0 \\
über & 0 & 7 & 0 & 0 \\
um & 0 & 1 & 0 & 0 \\
unter & 2 & 0 & 0 & 0 \\
von & 0 & 0 & 1 & 0 \\
vor & 0 & 0 & 0 & 0 \\
zu & 6 & 0 & 0 & 0 \\
zwischen & 0 & 2 & 0 & 0 \\
\hline Gesamt & 45 & 37 & 7 & 2 \\
\hline
\end{tabular}

Da die Definition des Frames Cause_to_move_in_place das Kriterium enthält, dass sich die mit den KtE des KE BEwEGENDEs bezeichnete Entität bewegt, ,without undergoing unbounded translational motion“ (FrameNet 1.7, Cause_to_ move_in_place) und der Frame deshalb auch keine FE wie PATH oder GoAL enthält, muss er, um den jeweiligen Konstrukt-Frame der Konstrukte in (47)-(48) bilden zu können, um solche FE aus dem Konstruktions-Frame Motion erweitert werden.

Für andere lexikalische Bedeutungen allerdings erscheint es plausibler, dass das betreffende KtE durch den lexikalischen Frame Cause_to_move_in_place motiviert wird, was dazu führt, dass alle KtE vollständig durch diesen motiviert werden. Die lexikalischen Bedeutungen von drehen und wenden (beide turn.v) in den Belegen in (49) sind Beispiele dafür.

(49) a. Jutta machte ein Geräusch und [cause_to_move_in_place drehte] sich [〈Direction〉 in meine Richtung], die Augen geschlossen. (Goosen, Frank: Liegen lernen, Frankfurt am Main: Eichborn AG 2000, S. 74)

b. Ein paar Dutzend Gesichter [Cause_to_move_in_place wandten] sich zuerst [〈DIRECtion $\rangle$ in die Richtung der Stimme] und danach in die, wohin der 
kleine Mann mit der Fliege winkte. (Suter, Martin: Lila, Lila, Zürich: Diogenes 2004, S. 211)

Da es sich bei Cause_to_move_in_place um einen unrelatierten lexikalischen Frame handelt, erscheinen Instanzen wie diejenigen in (49) zunächst nicht wie Konstrukte der reflexiven Bewegungskonstruktion, eben weil sie vollständig durch diesen lexikalischen Frame motiviert werden. Die Frage danach, ob ein gegebener Beleg ein Konstrukt der zu untersuchenden Konstruktion enthält, kann zwar grundsätzlich über den lexikalischen Frame beantwortet werden, die Entscheidung darüber kann aber nicht auf die lexikalischen Bedeutungen aller LE, die diesen Frame evozieren, invariant übertragen werden. Hierfür ist eine Feindifferenzierung nach lexikalischen Bedeutungen notwendig. Warum Belege wie diejenigen in (49) dennoch Konstrukte der reflexiven Bewegungskonstruktion zeigen, diskutiere ich in Unterabschnitt 6.4.3.

Abgesehen von diesen Zusammenhängen bleiben nun noch die Ergebnisse der empirischen Analyse der Konstrukte der reflexiven Bewegungskonstruktion, in denen die KtE des KE WEG durch den Konstruktions-Frame einfach motiviert werden, zu interpretieren. Ein interessanter Aspekt ist hier die bereits erwähnte Verteilung der FE des Konstruktions-Frames, die diese Motivierung übernehmen. Die in Tabelle 6.4 dargestellte Verteilung der FE des Konstruktions-Frames zeigt für die einfache Motivierung des einzelnen KtE WEG - eine deutliche Präferenz für das FE Motion.GoAL, nach dem das FE Motion.PATH an zweiter Stelle folgt. Diese Ergebnisse bestätigen die Hypothese, dass die Kodierung von Argumenten mit einer ,GoAL'-Bedeutung in der Regel Vorrang vor anderen semantischen Aspekten einer ,Bewegung،, insbesondere vor Argumenten mit ,SouRCE'-Bedeutung, hat (vgl. Ikegami 1979; Bourdin 1997) und die auch aus frame-semantischer und konstruktionsgrammatischer Sicht als bestätigt angesehen werden kann (vgl. Stefanowitsch \& Rohde 2004; Stefanowitsch 2018). Die reflexive Bewegungskonstruktion fügt sich somit in die Reihe der Evidenzen für diese Hypothese ein.

Die einfache Motivierung eines KtE durch den Konstruktions-Frame stellt den deutlichsten Fall einer Frame-Anpassung (Busse 2012: 624-627) dar, auf die ich in Unterabschnitt 4.4.1 hingewiesen habe: Der lexikalische Frame ist nicht ausreichend, um den Konstrukt-Frame zu konstituieren und muss deshalb um ein FE des Konstruktions-Frames erweitert werden. Im Sinne der Frame-Theorie von Minsky (1975: 213) findet hier also ein Abgleichprozess (matching process) zwischen dem lexikalischen Frame und dem Konstruktions-Frame statt: Außer dem KtE, das durch den Konstruktions-Frame einfach motiviert wird, können alle anderen Strukturelemente der Konstruktion durch den lexikalischen Frame motiviert werden - der Konstruktions-Frame ist hierfür nicht erforderlich. Einzig das in dem 
lexikalischen Frame fehlende FE zur Motivierung des entsprechenden KtE muss durch den Konstruktions-Frame beigetragen werden.

Der erwähnte Abgleichprozess führt also zu einer (temporären) Erweiterung des lexikalischen Frames innerhalb des Konstrukt-Frames. Somit „verleiht die Konstruktion als solche dem Konstrukt eine (zusätzliche) spezifische Bedeutung.“ (Deppermann \& Elstermann 2008: 104). ${ }^{61}$ Dieser neu entstandene KonstruktFrame liefert Evidenz dafür, „that combinations of verb and construction can [...] evoke novel frames.“ (Goldberg 2010: 56). ${ }^{62}$ Etwas ausführlicher:

Verbs necessarily evoke established semantic frames. Constructions also evoke established semantic frames. On the other hand, while classes of verbs are related to argument structure constructions by general, abstract frames, particular verbs may be combined with argument structure constructions to designate novel events that do not evoke any pre-existing semantic frame. (Goldberg 2010: 58)

Unter einem solchen ,neuen' Konstrukt-Frame ist die Basis für das Koerzionspotenzial einer Konstruktion (Abschnitte 5.5 und 7.4) sowie ihre Produktivität (Abschnitte 5.6 und 7.5) $\mathrm{zu}$ verstehen.

\subsubsection{Doppelte Motivierung eines KtE}

Dass ein lexikalischer Frame und ein Konstruktions-Frame gleichermaßen an der Konstitution eines Konstrukt-Frames beteiligt sein können, hat die Analyse in Unterabschnitt 6.2.2 gezeigt: Innerhalb eines Konstrukt-Frames kann ein KtE durch den Konstruktions-Frame motiviert sein, während ein anderes durch einen unrelatierten lexikalischen Frame motiviert ist. Beide Frames können in einem Konstrukt-Frame darüber hinaus aber noch anders in Erscheinung treten, nämlich dann, wenn ihre beiden FE nicht zwei verschiedene KtE motivieren, sondern wenn sie in einem einzigen KtE zusammenkommen. Wird ein KtE durch ein FE des Konstruktions-Frames und ein FE des lexikalischen Frames gleichzeitig motiviert, liegt ein Fall von doppelter Motivierung vor. Doppelte Motivierungen können (wie

61 Dies geschieht allerdings - wie ich gezeigt habe - nicht ,[u]ngeachtet des semantischen Beitrags der in ein Konstrukt eingehenden lexikalischen Einheiten“ (Deppermann \& Elstermann 2008: 104), gerade dann, wenn der lexikalische Frame mit dem Konstruktions-Frame identisch ist.

62 Lakonischer formuliert Goldberg (2010: 56) es wie folgt: „[W]hile the combination of an abstract meaning associated with a general verb class, together with an argument structure predication does seem to require an established semantic frame, the more specific meanings that arise from the combination of an argument structure construction and a specific verb need not.“ 
einfache) nicht nur KtE, sondern auch KEE betreffen, worauf in ich Unterabschnitt 6.3.3 eingehe.

An der doppelten Motivierung eines KtE zeigt sich am eindrücklichsten die Nützlichkeit des Einbezugs der Theorie der konzeptuellen Integration (Unterabschnitt 4.1.3) in das konstruktionssemantische Modell. Im Sinne einer konzeptuellen Integration liegt bei der doppelten Motivierung eines KtE eine Fusion zweier FE vor, jeweils eines FE aus jedem Input, also lexikalischem Frame und Konstruktions-Frame (Unterabschnitt 4.4.2). Die beiden FE können dabei semantische Gemeinsamkeiten teilen, müssen dies aber nicht. Die Tatsache, dass sie gemeinsam ein KtE motivieren, ist Grund genug, davon auszugehen, dass es sich um Gegenstücke (counterparts) im Sinne der Theorie der konzeptuellen Integration handelt, wohlgemerkt stets auf Token-Ebene, also innerhalb eines KonstruktFrames. Damit ist jedoch nicht ausgesagt, dass beide FE auf Type-Ebene semantisch kompatibel im Sinne des Semantic Coherence Principle von Goldberg (1995: 50) sein müssen.

Abbildung 6.3 zeigt, wie die doppelte Motivierung eines KtE als konzeptuelle Integration schematisch dargestellt werden kann. Beide FE fusionieren zu einem neuen FE des Konstrukt-Frames, das der semantischen Motivierung eines einzelnen KtE dient. Die durchgezogene Linie zwischen den beiden FE in den Inputs markiert die Gegenstück-Relation.

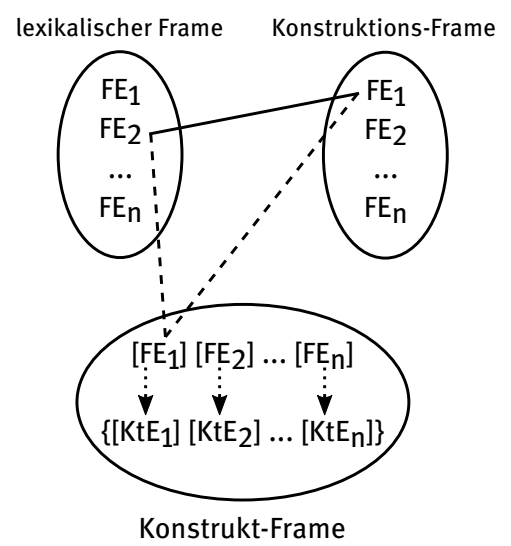

Abb. 6.3: Doppelte Motivierung eines KtE als konzeptuelle Integration

Auf einer Mikroebene zeigt sich hier ein Spezialfall des Koerzionspotenzials der Konstruktion, indem das FE, das der lexikalische Frame in den Konstrukt-Frame und damit die Motivierung des KtE einbringt, von einem FE des Konstruktions- 
Frames gewissermaßen ,umwickelt‘ (vgl. Pustejovsky \& Jezek 2008: 187, 203) wird. Fälle einer doppelten Motivierung müssen deshalb, ebenso wie alle diejenigen einer einfachen Motivierung, in die Messung des Koerzionspotenzials einer Konstruktion (Unterabschnitt 7.4.1) eingehen, denn sie entsprechen einer coercion by enrichment im Sinne von Audring \& Booij (2016: 629-631). Die doppelte Motivierung eines KtE als Etablierung einer Gegenstück-Relation zwischen zwei FE aus lexikalischem Frame und Konstruktions-Frame kann gleichzeitig ein Indiz für eine konzeptuelle Nähe der beiden Frames ausdrücken: ${ }^{63}$ „[T]wo concepts are conceptually close to the extent that they share semantic properties (e.g., two verbs are closer if they share a common tense, mood, subject, object, or topic)“ (Haiman 1983: 783). Der Koerzionseffekt, den der Konstruktions-Frame auf den lexikalischen Frame ausübt, ist damit für die semantische Motivierung eines KtE schwächer als bei dessen einfacher semantischer Motivierung durch den KonstruktionsFrame (vgl. Unterabschnitt 7.4.1).

Für die reflexive Bewegungskonstruktion sind in meiner Datenauswahl insgesamt 91 Konstrukte belegt, in denen eine doppelte Motivierung der KtE des KE WEG vorliegt. Wie bereits bei der einfachen Motivierung eines einzelnen KtE durch den Konstruktions-Frame (Unterabschnitt 6.2.2) betrifft auch die doppelte Motivierung vorrangig die KtE ebendieses KE. Ebenfalls wie bei der einfachen Motivierung eines einzelnen KtE durch den Konstruktions-Frame betrifft die doppelte Motivierung die FE Motion.Source, Motion.PATH, Motion.GoAL und Motion.Direction, die gemeinsam mit einem FE des lexikalischen Frames das jeweilige KtE motivieren. Es zeigt sich hier ebenso wie für die einfache Motivierung der KtE des KE WEG (vgl. Tabelle 6.4 in Unterabschnitt 6.2.2) eine deutliche Präferenz für das FE Motion.GoAL. Das FE Motion.DiRECTION ist nicht belegt. Die Tabellen 6.5 bis 6.7 zeigen absteigend nach der Frequenz für die drei belegten FE des Konstruktions-Frames die jeweiligen lexikalischen Frames und deren FE, die Teil der doppelten Motivierungen sind.

Wie bereits die einfache Motivierung eines KtE durch den KonstruktionsFrame ist auch die doppelte Motivierung abhängig vom lexikalischen Frame. Der Frame, für den hier die meisten Konstrukte belegt sind, ist Cause_to_amalgamate. Bei diesem lexikalischen Frame können die KtE des KE WEG mit dem FE Cause_to_ amalgamate.PART_2 und etwa dem FE Motion.GoAL des Konstruktions-Frames doppelt motiviert werden, wie die Belege in (50)-(52) mit den LE (ein)fädeln (etwa: merge.v), mischen und mengen (beide mix.v) zeigen.

63 Diese Nähe ist allerdings nicht mit der Frame-Nähe eines lexikalischen Frames zum Konstruktions-Frame (Abschnitt 5.4) zu verwechseln. Bei der doppelten Motivierung eines KtE liegt gerade keine Frame-Nähe vor, da stets ein unrelatierter lexikalischer Frame beteiligt ist. 
Tab. 6.5: Doppelte Motivierung der KtE des KE WEG der reflexiven Bewegungskonstruktion mit dem FE Motion.GoAL

\begin{tabular}{llrr}
\hline Lexikalischer Frame & FE & Konstrukte & Anteil \\
\hline Cause_to_amalgamate & PART_2 & 30 & $43,48 \%$ \\
Seeking & SoUGHT_ENTITY & 11 & $15,94 \%$ \\
Ingestion & INGESTIBLES & 4 & $5,80 \%$ \\
Manipulation & ENTITY & 4 & $5,80 \%$ \\
Desiring & EVENT & 3 & $4,35 \%$ \\
Others_situation_as_stimulus & SITUATION & 3 & $4,35 \%$ \\
Scouring & GROUND & 3 & $4,35 \%$ \\
Work & SALIENT_ENTITY & 3 & $4,35 \%$ \\
Giving & RECIPIENT & 2 & $2,90 \%$ \\
Attaching & GOAL & 1 & $1,45 \%$ \\
Burying & GOAL & 1 & $1,45 \%$ \\
Grinding & GOAL & 1 & $1,45 \%$ \\
Impact & IMPACTEE & 1 & $1,45 \%$ \\
Perception_experience & PHENOMENON & 1 & $1,45 \%$ \\
Smuggling & GOAL & 1 & $1,45 \%$ \\
\hline Gesamt & & 69 & $100,00 \%$ \\
\hline
\end{tabular}

Tab. 6.6: Doppelte Motivierung der KtE des KE WEG der reflexiven Bewegungskonstruktion mit dem FE Motion.PATH

\begin{tabular}{llrr}
\hline Lexikalischer Frame & FE & Konstrukte & Anteil \\
\hline Work & SALIENT_ENTITY & 6 & $40,00 \%$ \\
Ingestion & INGESTIBLES & 4 & $26,67 \%$ \\
Scouring & GROUND & 4 & $26,67 \%$ \\
Impact & IMPACTEE & 1 & $6,67 \%$ \\
\hline Gesamt & & 15 & $100,00 \%$ \\
\hline
\end{tabular}

Tab. 6.7: Doppelte Motivierung der KtE des KE WEG der reflexiven Bewegungskonstruktion mit dem FE Motion.SOURCE

\begin{tabular}{llrr}
\hline Lexikalischer Frame & FE & Konstrukte & Anteil \\
\hline Emptying & SOURCE & 3 & $42,86 \%$ \\
Assistance & HELPER & 1 & $14,29 \%$ \\
Filling & (SOURCE) & 1 & $14,29 \%$ \\
Manipulate_into_doing & RESULtING_ACTION & 1 & $14,29 \%$ \\
Smuggling & SOURCE & 1 & $14,29 \%$ \\
\hline Gesamt & & 7 & $100,00 \%$ \\
\hline
\end{tabular}


(50) a. Ein Lied singt sie zusammen mit Armand, [Cause_to_amalgamate fädelt] sich [PART_2 in seine Töne ${ }^{\text {GOAL}}$ ], und er spielt sie an, fällt vor ihr auf die Knie, kann ihr so unter den Rock gucken. (Dölling, Beate: Hör auf zu trommeln, Herz, Weinheim: Beltz \& Gelberg 2003, S. 205)

b. Im Regen [Cause_to_amalgamate fädelte] sich das Taxi mit wütenden Geräuschen [PART_2 in die Wagenschlange auf der Stadtautobahn GOAL] ein. (Riedel, Susanne: Eine Frau aus Amerika, Berlin: Berlin Verlag 2003, S. 15)

(51) a. Stimmen [Cause_to_amalgamate mischten] sich [PART_2 in das Rattern GoAL], Worte, Sätze, die sie nicht verstand, weil ihr Kopf so verschwommen war. (Pressler, Mirjam: Malka Mai, Weinheim Basel: Beltz \& Gelberg 2001, S. 248)

b. Die beiden Frauen [Cause_to_amalgamate mischen] sich [РART_2 unter die Zuschauertraube ${ }^{\text {GoAL }}$ ]. (Krausser, Helmut: Eros, Köln: DuMont 2006, S. 131)

c. Unverständliches Genuschel [Cause_to_amalgamate mischte] sich [PART_2 zwischen einzelne Schluchzer GoAL], es war unmöglich zu sagen, ob das Dienstmädchen aus Rührung, Empörung oder Eifersucht weinte. (Düffel, John von: Vom Wasser, München: dtv 2006, S. 206)

(52) Lichtgarben und Wolkenschatten [Cause_to_amalgamate mengen] sich [PART_2 in den Strom, der in seiner Meeresgrüne schäumend aufblitzt GoAL]. (Düffel, John von: Vom Wasser, München: dtv 2006, S. 69)

Ebenfalls in höherer Frequenz für das FE Motion.GoAL belegt ist der lexikalische Frame Seeking. In die Motivierung der KtE des KE WEG geht dessen FE Seeking.Sought_EnTITY ein. Die Belege in (53)-(54) sind Beispiele für die LE tappen und tasten (beide grope.v).

(53) Die jetzt 30-köpfige Truppe der Poststaatsfarmer [seeking tappt] sich nach Hörensagen [Sought_ENTITy an EU-Vorschriften GoAL] heran, entsorgt die Gülle bodenschonend, gibt den Schweinen mehr Kräuter als Hormone. (Die Zeit, 16.03.2000, Nr. 12)

(54) a. Dabei [seeking tastet] sich die Elektronik [Sought_entity an jenen Punkt heran, an dem die Motordrehzahl zu sinken beginnt GoaL]. (Die Zeit, 06.04.2000, Nr. 15)

b. Langsam wagen sie sich heran, [seeking tasten] sich [Sought_EnTity auf meinen vom Schmerz durchwaberten Rücken GoAL]; die ungeheuerliche Berührung, als testeten sie, ob oder auf welche köstliche Weise 
ihre Beine wohl einsinken in diesem seltenen eßbaren Grund. (Braun, Marcus: Hochzeitsvorbereitungen, Berlin: Berlin Verlag 2003, S. 113)

c. Die Frau nimmt nicht den Fahrstuhl, sondern [seeking tastet] sich über die Treppen hinauf [Sought_entity in den sechsten Stock GoAL], vorbei an den schlafenden Bettlern, die der heilige Martin vergaß. (Venske, Regula: Marthes Vision, Frankfurt am Main: Eichborn Verlag 2006, S. 184)

d. Er ruckelte und scharrte, bis er die Schaufel voll glaubte, [seeking tastete] sich rückwärts gehend [Sought_Entity $z u$ den Fahrrädern ${ }^{\text {GOAL }}$ ] zurück, richtete sich auf, drehte sich wieder um die eigene Achse und ging, aufrecht nun und die volle Schaufel waagrecht an einer Seite tragend, an den Kisten und Koffern vorbei, die zwei Stufen zur Heizung hinunter. (Widmer, Urs: Das Buch des Vaters, Zürich: Diogenes 2004, S. 167)

Semantische Motivierungen der KtE des KE WEG mit dem lexikalischen Frame Scouring sind nicht nur für ein einziges FE des Konstruktions-Frames Motion belegt, sondern einerseits für das FE Motion.GoAL und andererseits für das FE Motion.PATH. Als FE des lexikalischen Frames tritt Scouring.Ground hinzu. Die Belege in (55) und (56) sind jeweils Beispiele für die LE graben (etwa: rummage.v) und wühlen (rifle.v).

a. Sharon [scouring gräbt] sich tiefer [Ground in den Sand GoAL], woraus ich schließe, daß sie ihren eigenen Gefühlen schon sehr nah gekommen ist. (Riedel, Susanne: Eine Frau aus Amerika, Berlin: Berlin Verlag 2003, S. 85)

b. Er klammerte sich so fest daran, dass seine kurzen Fingernägel sich [Ground in ihre Haut GoAL] [scouring gruben]. (Funke, Cornelia: Tintenherz, Hamburg: Cecilie Dressler Verlag 2003, S. 320)

(56) a. Lastwagen [scouring wühlen] sich [GRound durch das weiche Erdreich $\mathrm{PATH}$ ], kippen ockerbraunen Sand obenauf. (Die Zeit, 24.02.2000, Nr. 9)

b. Unermüdlich [scouring wühlten] sich ihre geschmeidigen Finger [GRound durch das schwarze, schattenhafte Fleisch der erschlagenen Forellen $\mathrm{PATH}^{\mathrm{A}}$, während das Gewitter immer wieder aufglomm und verlosch und schließlich in schmutziger, schummriger Dunkelheit unterging. (Düffel, John von: Vom Wasser, München: dtv 2006, S. 191)

$\mathrm{Ob}$ in einem gegebenen Konstrukt eine einfache oder doppelte Motivierung eines KtE des KE WEG vorliegt, kann zwar in einigen Fällen an dem lexikalischen Fra- 
me erkannt werden, manche lexikalischen Frames aber erlauben beide Varianten. Ein Vergleich von Tabelle 6.3 in Unterabschnitt 6.2.2 mit den Tabellen 6.5 bis 6.7 zeigt, dass für in einigen Fällen für denselben lexikalischen Frame sowohl eine einfache Motivierung des KtE des KE WEG durch den Konstruktions-Frame belegt ist als auch eine doppelte. $\mathrm{Zu}$ den lexikalischen Frames, die dies betrifft, gehört der soeben diskutierte Seeking. Die folgenden drei Belegpaare (57)-(59) für die lexikalischen Frames Ingestion, Seeking und Work für die LE saufen (etwa: drink.v) und fressen (etwa: eat.v), tasten (grope.v) sowie arbeiten (work.v) sollen verdeutlichen, dass die Entscheidung darüber, welche Variante vorliegt, nicht allein über den lexikalischen Frame getroffen werden kann, sondern letztlich über die Konstruktbedeutung getroffen werden muss. Abhängig ist sie in diesen Fällen stets von der konkreten Instanziierung des KE WEG.

(57) a. Es war allen Anwesenden nachzufühlen; von unschuldigem Frohsinn war nichts zu spüren, die Abende zogen sich dahin, der Steuereintreiber und der Chirurg entschuldigten sich schon früh, um nicht wieder in den Genuß von Gratiskonsultationen zu kommen, der Priester gab seine zwei Witze zum besten, und der Rest lästerte über das Essen und [Ingestion soff] sich [unter den Tisch GoAL], weil selbst die Konjunktur als Thema längst ausgeschöpft war. (Schrott, Raoul: Tristan da Cunha oder die Hälfte der Erde; Hanser Verlag 2003, S. 196)

b. Ginster [Ingestion frißt] sich mit knorrigen Fingern [Ingestibles in den Felsblock ${ }^{\mathrm{GOAL}}$ ] und lächelt unter Lichtflecken wie ein bekränzter Gott. (Riedel, Susanne: Eine Frau aus Amerika, Berlin: Berlin Verlag 2003, S. 184)

(58) a. Er trat auf Treppen, die unter Getöse nachgaben, durchquerte abschüssige Räume, [seeking tastete] sich [durch lichtlose Gänge ${ }^{\mathrm{PATH}}$ ]. (Glavinic, Thomas: Die Arbeit der Nacht, München Wien: Carl Hanser Verlag 2006, S. 49)

b. Schiller [seeking tastet] sich [Sought_Entity an den Urschmerz des Bewußtseins GoAL] heran, an jenen Augenblick, da das erwachende Bewußtsein die unmittelbare Leichtigkeit des Seins, die traumwandlerische Sicherheit der natürlichen Lebensvollzüge, die Unbefangenheit verliert. (Safranski, Rüdiger: Friedrich Schiller, München Wien: Carl Hanser 2004, S. 419)

(59) a. Irgendein ferner Schmerz [work arbeitet] sich [auf mich GoAL] zu, ich sehe ihn kommen. (Genazino, Wilhelm: Die Liebesblödigkeit, München, Wien: Carl Hanser Verlag 2005, S. 108) 
b. Er [work arbeitete] sich [SALIENT_EnTITy durch ein kompliziertes Kreuzworträtsel $^{\mathrm{PATH}}$, hatte ein Bier aufgemacht und sich auf einen beschaulichen Abend eingestellt, als das Telefon läutete. (Glavinic, Thomas: Die Arbeit der Nacht, München Wien: Carl Hanser Verlag 2006, S. 381)

In (57-a) bezeichnet das KtE allein eindeutig den ,Weg‘ der ,Bewegung‘, die PP unter den Tisch kann nicht gleichzeitig auf den ,Gegenstand' der ,Nahrungsaufnahme' referieren. Dies ist bei (57-b) anders: Hier referiert in den Felsblock (metaphorisch) auf den ,Gegenstand', der zumindest teilweise ,gefressen' wird. In (58-a) ist das KtE durch lichtlose Gänge kaum als Entität zu sehen, die Gegenstand des ,Such'-Ereignisses ist. In (58-b) wiederum bezeichnet an den Urschmerz des Bewußtseins genau dies. Schließlich ist auf mich zu in (59-a) lediglich als ,Endpunkt ${ }^{*}$ der ,Bewegung‘ zu verstehen, während in (59-b) durch ein kompliziertes Kreuzworträtsel in jedem Fall Teil des Ereignisses des ,Arbeitens‘ sein muss.

\subsubsection{Eine Kritik an Goldbergs Semantic Coherence Principle}

Die Beobachtungen der Unterabschnitte 6.2.1 bis 6.2.3 sollen die empirischen Analysen zur Motivierung der KtE des KE WEG der reflexiven Bewegungskonstruktion zunächst abschließen. Bevor ich mich in Abschnitt 6.3 der Motivierung des KEE zuwende, sei noch ein theoretisches Konzept diskutiert, das bei der Analyse doppelt motivierter KtE nicht ohne Beachtung bleiben darf. Die doppelte Motivierung eines KtE ist in der Konstruktionsgrammatik grundsätzlich kein unbekanntes Phänomen. Ein ähnlicher Prozess wird bereits von Goldberg (1995: 50-52) als Fusion von Partizipantenrollen und Argumentrollen diskutiert. Dabei stellt sie zwei Prinzipien auf, denen eine solche Fusion folgen muss, ${ }^{64}$ eines davon ist das Semantic Coherence Principle: 65

64 Mandelblit \& Fauconnier (2000: 167, 169-170) gehen von dem vergleichbaren Prinzip aus, dass die Entstehung eines Blends nur möglich ist, wenn eine Gegenstück-Relation zwischen den Inputs oder zumindest eine Ähnlichkeit zwischen diesen vorliegt. Ohne empirische Befunde ist dies allerdings, ebenso wie Goldbergs Semantic Coherence Principle, grundsätzlich infrage zu stellen.

65 Das zweite Prinzip, das Correspondence Principle, besagt, dass nur profilierte Partizipantenund Argumentrollen fusionieren dürfen (vgl. Goldberg 1995: 50-52, 2006: 39-40). Diese könnten in etwa mit Nicht-Kern-FE im Sinne von FrameNet verglichen werden. Freilich ist dieses Prinzip ebenso wenig empirisch gesichert wie das Semantic Coherence Principle, es würde daher eine eigene Untersuchung benötigen, die ich an dieser Stelle nicht leisten kann. 
The Semantic Coherence Principle: Only roles which are semantically compatible can be fused. Two roles $r_{1}$ and $r_{2}$ are semantically compatible if either $r_{1}$ can be construed as an instance of $r_{2}$, or $r_{1}$ [sic!] can be construed as an instance of $r_{1}$. For example, the kicker participant of the kick frame may be fused with the agent role of the ditransitive construction because the kicker role can be construed as an instance of the agent role. (Goldberg 1995: 50)

\section{Eine erweiterte Bestimmung dieses Prinzips lautet bei ihr wie folgt:}

The Semantic Coherence Principle ensures that the participant role of the verb and the argument role of the construction must be semantically compatible. In particular, the more specific participant role of the verb must be construable as an instance of the more general argument role. General categorization processes are responsible for this categorization task and it is always operative. (Goldberg 2006: 40)66

Abgesehen davon, dass diesem Prinzip die grundsätzliche Annahme eines Unterschieds der Abstraktheit zwischen Konstruktions-Frame und lexikalischem Frame zugrunde liegt, die ich an Goldbergs Modell bereits kritisiert und in Unterabschnitt 4.3.3 für das konstruktionssemantische Modell in Zweifel gezogen habe, bietet es sich an, die empirischen Befunde, die ich für die doppelte Motivierung der KtE des KE WEG der reflexiven Bewegungskonstruktion erhoben habe, im Lichte des Semantic Coherence Principle zu betrachten. ${ }^{67}$ Dies betrifft zum einen die Ebene der semantischen Motivierung eines einzelnen KtE durch zwei FE, lässt sich jedoch auch genereller auf die Kompatibilität von lexikalischem Frame und Konstruktions-Frame beziehen:

Since the argument roles of a construction are defined with reference to the meaning of that construction, and the participant roles of a verb are defined with reference to the framesemantic meaning of the verb, the semantic coherence principle can be more generally understood as requiring that the semantic frame evoked by the verb be semantically compatible with the meaning of the construction. (Perek 2015: 24)

Goldbergs Kriterium der Deutbarkeit einer Partizipantenrolle als Instanz einer Argumentrolle lässt sich konstruktionssemantisch wie folgt reformulieren: Wenn

66 Goldberg \& Jackendoff (2004: 550) betonen, „that the principle is stated as a constraint on the combination of roles in a clause, not as a real-world constraint on the referent of those roles.“ Dies allerdings ändert nichts an meiner Kritik, da es eben genau darum geht, dass zwei auch semantisch miteinander inkompatible FE ein KtE doppelt motivieren können.

67 Gleichzeitig ist dies ein empirischer Prüfstein für Goldbergs These, dass lexikalische Bedeutung (bzw. lexikalischer Frame) und ,Konstruktionsbedeutung، (bzw. Konstruktions-Frame) immer in einer (mindestens kausalen) Relation zueinander stehen müssen: der Causal Relation Hypothesis oder (in erweiterter Form) der Force Dynamic Relation Hypothesis (vgl. Goldberg 1995: 61-65, 1997: 387-394). 
sich das Semantic Coherence Principle bestätigt, müsste in Fällen der doppelten Motivierung eines KtE das FE des lexikalischen Frames als spezifischere Instanz des FE des Konstruktions-Frames zu verstehen sein.

Die Belege der reflexiven Bewegungskonstruktion, von denen ich in Unterabschnitt 6.2.3 nur einen kleinen Teil analysiert habe, bieten eine Grundlage für die Überprüfung dieser Hypothese. Man betrachte dazu die Tabellen 6.5 bis 6.7, in denen alle doppelten Motivierungen mit den FE Motion.GoAL (Tabelle 6.5), Motion.PATH (Tabelle 6.6) und Motion.SourcE (Tabelle 6.7) den FE entsprechender lexikalischer Frames gegenübergestellt sind. Diese Ergebnisse zeigen, dass eine Interpretation nach dem Semantic Coherence Principle Schwierigkeiten bereitet: Anders als es das Semantic Coherence Principle voraussagt, sind die FE der lexikalischen Frames kaum als spezifischere Instanzen der FE des KonstruktionsFrames $\mathrm{zu}$ verstehen. ${ }^{68}$ Am ehesten scheint eine solche Interpretation noch für den lexikalischen Frame Scouring möglich. Dessen FE Scouring.Ground ist bei FrameNet wie folgt definiert: „The location that the SEARCHER covers in looking for the Sought_ENTITY.“ (FrameNet 1.7, Scouring). Dieses FE scheint kompatibel mit den FE Motion.GoAL, Motion.PATH und Motion.SourCE, die in FrameNet wie folgt definiert sind.

- „The GoAL is the location the ThEme ends up in.“ (FrameNet 1.7, Motion)

- „The PATH refers to (a part of) the ground over which the THEME travels or to a landmark by which the THEmE travels.“ (FrameNet 1.7, Motion)

- „The Source is the location the THEME occupies initially before its change of location.“ (FrameNet 1.7, Motion)

- „This FE [DIRECTION, A.W.] is used for expressions that indicate motion along a line from the deictic center towards a reference point (which may be implicit) that is neither the GoAL of the posture change nor a landmark along the way of the moving part of the body. Often DIRECTION is defined with reference to the canonical orientation of the Protagonist, or the orientation imposed by an implicit observer.“ (FrameNet 1.7, Motion)

68 Iwata (2008: 124) führt ebenso Argumente gegen das Semantic Coherence Principle an, wonach sich die Kompatibilität von ,Konstruktionsbedeutung ( kalischer Bedeutung (bzw. lexikalischem Frame) eher daraus ergeben sollte, ob die ,Konstruktbedeutung ( bzw. der Konstrukt-Frame) als Ganzes als eine spezifischere Instanz der ,Konstruktionsbedeutung، (des Konstruktions-Frames) zu verstehen ist. Dies allerdings löst das Problem kaum, da relatierte lexikalische Frames mit einer negativen Frame-Nähe (wie etwa Cause_motion mit -1) gerade keine spezifischeren, sondern abstraktere Instanzen des Konstruktions-Frames darstellen (vgl. Unterabschnitt 6.2.1). 
Für diese Kompatibilität dieser FE sorgt insbesondere der Hinweis auf einen ,ground' im FE Motion.PATH (der sich ja auch im Namen des FE Scouring.GROUND widerspiegelt) sowie die Parallele zu einer ,location‘ im FE Motion.GoAL. Weitere mögliche Parallelen zwischen FE lexikalischer Frames und den vier genannten FE des Frames Motion sind in Tabelle 6.8 dargestellt.

Tab. 6.8: Mögliche Parallelen zwischen FE des Konstruktions-Frames Motion und für die reflexive Bewegungskonstruktion belegte FE lexikalischer Frames

\begin{tabular}{|c|c|c|c|}
\hline Motion.GoAL & Motion.PATH & Motion.SoURCE & Motion.DIRECTION \\
\hline \multicolumn{4}{|l|}{ Attaching.GoAL } \\
\hline \multicolumn{4}{|l|}{ Burying.GoAL } \\
\hline & & Emptying.SouRCE & \\
\hline & & Filling.〈SOURCE) & \\
\hline \multicolumn{4}{|l|}{ Grinding.GOAL } \\
\hline Scouring.GROUND & Scouring.GROUND & & \\
\hline Smuggling.GoAL & & Smuggling.SouRCE & \\
\hline
\end{tabular}

Für zahlreiche lexikalische Frames sind solche Parallelen allerdings nicht zu beobachten. So lautet etwa die Definition des FE Work.SALIENT_ENTITY wie folgt: „An entity that is centrally involved in the GoAL that the AGENT is attempting to achieve.“ (FrameNet 1.7, Work). Die des FE Cause_to_amalgamate.PART_2 lautet: „This FE identifies the second of two PARTs mentioned. It is usually a PP Complement.“ (FrameNet 1.7, Cause_to_amalgamate). Ebenfalls kaum vorstellbar ist eine Parallele zum FE Ingestion.INGESTIBLES: „The INGESTIBLES are the entities that are being consumed by the INGESTOR. "(FrameNet 1.7, Ingestion). Diese FE dieser lexikalischen Frames sind kaum als spezifischere Instanzen der mit ihnen doppelte Motivierungen eingehenden FE des Konstruktions-Frames zu deuten, was auch daran liegt, dass Goldberg keine Kriterien für eine solche Instanzbeziehung formuliert und diese somit kaum objektiv zu entscheiden ist. Die FE der lexikalischen Frames sind mit den FE des Konstruktions-Frames nach Goldbergs Semantic Coherence Principle demnach inkompatibel, ihre Fusion ist aber dennoch empirisch als doppelte Motivierung von KtE nachzuweisen.

Wie lässt sich mit diesem Umstand umgehen? Aus gebrauchsbasierter Perspektive und insbesondere aus Sicht der Produktivität der Konstruktion (Abschnitt 5.6) ist Goldbergs Semantic Coherence Principle fragwürdig. Es hätte zur Konsequenz, dass ,neue und ,kreative' Konstrukte einer Konstruktion nur dann entstehen können, wenn die semantischen Eigenschaften der Konstruktion mit denen einer LE, die potenziell eines ihrer KE instanziieren kann, semantisch ein- 
ander angeglichen sind. Müssten lexikalischer Frame und Konstruktions-Frame auch hinsichtlich der semantischen Motivierung von KE stets - nach welchen Kriterien auch immer - kompatibel sein, würde dies nicht nur die Kreativität im Sprachgebrauch stark einschränken, sondern ebenso die Leistung des Konstruktions-Frames schmälern. Im Extremfall wären ausschließlich relatierte lexikalische Frames, also solche, die zum Konstruktions-Frame in Frame-Nähe (Abschnitt 5.4) stehen, möglich und zudem noch solche mit einer hohen Prototypikalität (z.B. einer Frame-Nähe von +1 oder +2 ), da sie die zum KonstruktionsFrame semantisch kompatibelsten lexikalischen Frames darstellen.

Die aus konstruktionssemantischer Sicht interessanten Fälle sind aber gerade diejenigen, in denen lexikalischer Frame und Konstruktions-Frame nicht in Frame-Nähe zueinander stehen und in denen deutliche Koerzionseffekte sichtbar werden, die die Leistung des Konstruktions-Frames deutlich erkennbar werden lassen (vgl. Abschnitt 7.4). Dies räumt Goldberg (2010: 53), wie das Zitat in Unterabschnitt 6.2.2 gezeigt hat, gewissermaßen selbst ein. ${ }^{69}$ Damit ist klar, dass die Annahme einer notwendigen Kompatibilität zwischen lexikalischem Frame und Konstruktions-Frame nicht nur für die semantische Motivierung einzelner KtE, sondern auch für die beiden Frame-Typen generell kaum aufrechtzuerhalten ist. Das Semantic Coherence Principle kann demnach kaum als „always operative“ (Goldberg 2005b: 226, 2006: 40) gelten.

Aus gebrauchsbasierter Perspektive lässt sich darüber hinaus ein weiteres Argument gegen die Annahme eines Semantic Coherence Principle vorbringen. So stellt Bybee (2010: 136-138) am Beispiel der phonologischen Kontraktion von Subjekt und Auxiliar im Englischen (z.B. I will zu I'll) fest, dass jene Kontraktion wesentlich von der Frequenz der Kookkurrenz beider Einheiten, nicht primär von einer semantischen Kompatibilität abhängt (vgl. auch Bybee 2002: 125):

Since the subject and the auxiliary are traditionally assigned to different constituents and since their combination evinces no semantic coherence or relevance, it is only their frequency of co-occurrence that drives them to fuse into a single phonological unit. (Bybee 2010: 137)

Analog lässt sich auch für die Fusionen, die eine doppelte semantische Motivierung hervorbringen, ein gewisser Frequenzeffekt beobachten: Wie aus den Tabellen 6.5 bis 6.7 hervorgeht, sind 91 von insgesamt 1.011 Konstrukten der reflexiven

69 Eine ähnliche Schlussfolgerung lässt sich in Begriffen der Kompositionalität von Konstruktions-Frame und lexikalischem Frame ziehen: „Crucially, composition is not just a matter of putting together pieces of the 'same kind' of content into a single homogeneous structure. Compositionality looks more homogeneous, or at least more unified, if we recognize the range and diversity of the units to be combined.“ (Sweetser 1999: 156). 
Bewegungskonstruktion von einer doppelten Motivierung eines KtE betroffen. Es handelt sich damit also keineswegs um ein Randphänomen.

Selbstverständlich sind Frequenzdaten allein kein direkter Grund zur Annahme, dass Fusionen wie diejenigen, die eine doppelte Motivierung eines KtE erzeugen, ohne semantische Kompatibilität auskommen. Es ist vorstellbar, dass Konstrukte, die eine solche Fusion enthalten, in vielen Fällen gerade aus semantischen Gründen produziert werden. An der reflexiven Bewegungskonstruktion wird dies daran sichtbar, dass viele lexikalische Frames durch den Konstruktions-Frame insofern koerziert werden, dass der ursprüngliche lexikalische Frame zwar in Form mindestens eines FE (das das KtE des KE BEwEGENDES motiviert) im Konstrukt-Frame ,übrig‘ bleibt, aber eine Frame-Anpassung durch den Konstruktions-Frame erfährt, die im Einfügen von dessen FE in den Konstrukt-Frame besteht (sofern es sich um einen unrelatierten lexikalischen Frame handelt). Ein solcher Koerzionseffekt liegt aber gerade dann vor, „when lexical and grammatical meanings are not compatible“ (Bybee 2010: 186). Die Inkompatibilität von Frames und ihren FE steht dem nicht im Wege, sondern ist ein wesentlicher Motor für Koerzion. Die hierbei entstehende Anreicherung des lexikalischen Frames kann grundsätzlich aus einem semantischen Bezeichnungsbedarf resultieren, dessen Ergebnis die Konstitution eines Konstrukt-Frames ist, ${ }^{70}$ der - als Token-Frame - einer in Unterabschnitt 4.4.1 diskutierten, wichtigen Eigenschaft von Frames Rechnung trägt, denn „Frames sind [...] nicht nur anpassungsfähig, sondern stets auch anpassungsbedürftig“ (Busse 2012: 625). Ebendieser semantische Anpassungsbedarf führt dazu, dass ein lexikalischer Frame, der für einen gegebenen Kontext zur Konstitution eines Konstrukt-Frames allein nicht ausreichend ist, durch FE des Konstruktions-Frames angepasst wird. Eine a priori gegebene semantische Kompatibilität im Sinne des Semantic Coherence Principle ist dafür nicht nötig, im Gegenteil: Sie würde das Zustandekommen von Koerzionseffekten gar nicht erlauben.

\subsection{Konstruktionsevozierende Elemente und Frame-Elemente}

Die zu Beginn dieses Kapitels diskutierte und in Tabelle 6.1 dargestellte Gegenüberstellung der Strukturelemente von Frames und Konstruktionen (nach LeeGoldman \& Petruck 2018: 36) setzt KEE mit LE gleich. Wie erwähnt, beruht die-

70 In den Worten von Fauconnier (1997: 173): „The general driving force behind this phenomenon [Konstruktionen bzw. Konstrukte als Ergebnisse von Blending-Prozessen, A.W.] is the linguistic pressure to represent complex integrations of events by making maximum use of existing grammatical constructions.“ 
se Gleichsetzung auf einer zunächst rein terminologischen Parallele, die sich um den Begriff des ,Evozierens' dreht und dabei mindestens zwei Fragen aufwirft. Zunächst ist fraglich, ob KEE tatsächlich einen ähnlichen Status wie LE haben, ob sie also (neben Konstruktionen) selbst auch Frames evozieren können. Wird dies mit Ja beantwortet, ist damit die Frage verbunden, ob ihnen dann tatsächlich keine semantische Motivierung durch einen Frame zukommt, da sie dann ja ähnlich wie die KtE des KE EREIGNIS als lexikalische Targets anzusehen wären, die zwar Frames evozieren, selbst aber nicht durch Frames motiviert sind (dazu auch Unterabschnitt 7.3.1). Bevor ich erstere Frage in Unterabschnitt 8.3.1 adressiere, möchte ich in diesem Abschnitt - für die reflexive Bewegungskonstruktion - argumentieren, dass KEE durchaus durch Frames semantisch motiviert sein können. Ich möchte zeigen, dass ein KEE nicht allein ,indicates any lexically-limited material (if any)“ (Fillmore, Lee-Goldman \& Rhomieux 2012: 323), sondern selbst durch FE semantisch motiviert sein kann.

Die Annahme, dass das KEE der reflexiven Bewegungskonstruktion (und analog dasjenige der reflexiven Partikelverbkonstruktion und der reflexiven WegKonstruktion) semantisch motiviert ist, ihm also ein FE des lexikalischen Frames oder des Konstruktions-Frames zugeordnet werden kann, stellt eine Konstruktionssemantik vor Herausforderungen. Da es sich bei den drei untersuchten Konstruktionen um Reflexivkonstruktionen handelt und das KEE somit ein Reflexivum ist, steht zur Diskussion, ob diesem Reflexivum überhaupt plausibel FE zugeordnet werden können und wenn ja, welche FE dies sein können und welchem Frame sie entstammen, dem lexikalischen Frame oder dem Konstruktions-Frame.

Diese Frage ist theoretisch und empirisch nicht abschließend geklärt (vgl. auch Kaufmann 2004: 15-17, 219-222). Geht man davon aus, dass das Reflexivum und sein Antezedens (also das KtE des KE BEWEGENDES) ,in der Regel in zwei verschiedenen semantischen Rollen“ (Zifonun, Hoffmann \& Strecker 1997: 1359) kodiert werden (vgl. auch Zifonun 2003: 9; Duden 2016: 407), legt dies eine Arbeitsteilung zwischen zwei FE nahe. Diese Annahme ist jedoch nicht in dieser Pauschalität zu halten. So weist Zifonun (2003: 17) darauf hin, dass „bei nichtreferentiellen Verwendungen diese Unterscheidbarkeit der beiden Rollen oder auch nur Facetten des Partizipanten in unterschiedlich starkem Maße abgebaut“ ist. Eine klarere Grenze ziehen Buscha (1982: 170-171), Eroms (2000: 307, 411), von Polenz (2008: 126-129) und auch die Duden-Grammatik (Duden 2016: 408): Sie gehen davon aus, dass Reflexiva bei ,echt reflexiven ${ }^{6}$ Verben $^{71}$ „nicht als Ergänzungen, sondern als Bestandteil des Prädikatsausdrucks“ (von Polenz 2008: 127)

71 Ich halte mich hier an die z.B. bei Hentschel \& Weydt (2013: 59-60) und in Duden (2016: 410) zu findende Terminologie. In der Reflexivitätsforschung ist sie freilich nicht einheitlich, statt ,echt reflexiv‘ finden sich dort unter anderem auch die Begriffe pseudo-reflexiv (Buscha 1972: 157), re- 
bzw. „Verbteil“ (Eroms 2000: 307) eingestuft werden müssen und damit keine semantische Rolle tragen. Das Reflexivum ist in diesen Fällen also „Teil des verbalen Lexems und gehört zur Lexikoneintragung der Verben.“ (Buscha 1982: 171).72

Bei ,reflexiv gebrauchten ${ }^{6}$ Verben ${ }^{73}$ „entspricht dagegen dem echten Reflexivpronomen sich/mich usw. (das durch selbst ergänzbar ist) im Satzinhalt eine Referenzstelle, die mit der ersten Referenzstelle der gleichen Prädikation bezugsidentisch ist“" (von Polenz 2008: 127). ${ }^{74}$ Auf derselben Linie nimmt auch Duden (2016: 408) an, dass eine Arbeitsteilung zwischen zwei semantischen Rollen nur möglich ist, wenn es sich um ,reflexiv gebrauchte' Verben handelt, bei inhärent reflexiven Verben jedoch nicht. Mit Verweis auf Primus (2012: 6-7) hält Hoherz (2017: 304) fest, dass bei ,echt reflexiven“ Verben „der sich-Marker keinen lexikalischen Wert hat und daher keine explizite semantische Rolle übernehmen kann“, wohingegen dies bei ,reflexiv gebrauchten' Verben durchaus der Fall sein kann. ${ }^{75}$

flexiv (Buscha 1982: 171), lexikalisch (Reis 1982: 4), medial (Haider 1985: 246-248; Ágel 1997b: 71; Welke 2005: 229-259, 2011: 308, 2019: 406-426), nicht-referenziell (Kunze 1997: 88; Zifonun 2003: 68-69) oder mitszenierend (Ágel 2017: 334).

72 Obwohl er dem Reflexivum bei ,echt reflexiven` Verben keine semantische Rolle zuweist, zählt Duden (2016: 410-411) es nicht zum Verb, sondern betrachtet es im Sinne einer Trennung von syntaktischer und semantischer Valenz als syntaktische, aber nicht semantische Ergänzung des Verbs.

73 Auch hier bleibe ich bei der Terminologie von Hentschel \& Weydt (2013: 59-60) und Duden (2016: 407). Alternative Begriffe dafür sind reflexive Konstruktion (Buscha 1982: 196), anaphorisch (Reis 1982: 3; Haider 1985: 242-244), reflexiv (Ágel 1997b: 70-71), koreferenziell (Kunze 1997: 89), reflexivierbar bzw. optional reflexiv (Zifonun 2003: 68-69) oder szenariokomplementierend (Ágel 2017: 334).

74 Hierbei ist allerdings zu berücksichtigen, dass die Anzahl der einem Prädikat zuzuordnenden Referenzstellen nicht mit der Anzahl der semantischen Rollen übereinstimmen muss, gerade bei Verben in Kombination mit Reflexiva (vgl. Geniušienè 1987: 44-47; Dux 2020: 28-29).

75 Dass diese Positionen jedoch nicht die einzige Lösung darstellen, wird bei einem Blick in weitere Arbeiten sichtbar: Schon Wagner (1977: 49-55) umgeht das Problem, indem er bei der Anwendung von Fillmores Kasusgrammatik auf eine Familie von Reflexivkonstruktionen lediglich mögliche semantische Rollenzuweisungen an das Antezendens betrachtet und das Reflexivum dabei außer Acht lässt. Kunze (1997: 96) geht bereits einen Schritt weiter, indem er die Frage, ob das Reflexivum eine semantische Rolle in einem Kasusrahmen besetzen kann, als Kriterium für die Unterscheidung von Typen von Reflexivkonstruktionen ansetzt. Ágel (1997a: 163) geht davon aus, dass Antezendens und Reflexivum (immer?) zwei verschiedene semantische Rollen tragen, elaboriert dies aber nicht weiter. Schließlich spricht Oya (2010: 228) in seiner Klassifikation reflexiver Verben lediglich für einen einzigen Typ dem Reflexivum eine semantische Rolle zu. Somit scheint mit Lehmann (2017: 73-74, 109-110, 123-124) davon auszugehen zu sein, dass die Zuweisung zweier unterschiedlicher semantischer Rollen (bzw. FE) ebenso wie die Frage nach der Referenzidentität des KEE mit dem KtE des KE BEWEGENDES nicht auf alle Reflexivkonstruktionen verallgemeinerungsfähig, sondern nur konstruktionsspezifisch anzunehmen ist. Auf die 
Ob eine entsprechende Arbeitsteilung zwischen zwei FE möglich ist, muss in Fällen der möglichen einfachen Motivierung des KEE durch den (relatierten) lexikalischen Frame betrachtet werden, aber auch in solchen, in denen ein unrelatierter lexikalischer Frame die Konstitution des Konstrukt-Frames gemeinsam mit dem Konstruktions-Frame leistet. Voraussetzung dafür ist freilich, dass überhaupt zwei FE existieren, die einem KtE des KE BEWEGENDES einerseits und dem KEE andererseits zugeordnet werden können. Für zahlreiche lexikalische Frames ist dies in der Tat nicht möglich, worauf ich in Unterabschnitt 6.3.1 eingehe.

Analog zu den drei Varianten der Motivierung von KtE in Abschnitt 6.2 unterscheide ich auch für KEE eine einfache Motivierung durch den lexikalischen Frame (Unterabschnitt 6.3.1), eine einfache Motivierung durch den KonstruktionsFrame (Unterabschnitt 6.3.2) sowie eine doppelte Motivierung durch beide FrameTypen (Unterabschnitt 6.3.3).

\subsubsection{Einfache Motivierung durch lexikalischen Frame}

Damit ein Reflexivum als KEE durch den lexikalischen Frame einfach motiviert werden kann, muss der dafür herangezogene Frame die angesprochene Arbeitsteilung zwischen dem KEE und dem KtE des KE BEWEGENDES ermöglichen, mit anderen Worten: Er muss über zwei unterschiedliche FE verfügen, die dem KtE des KE BEWEGENDES und dem KEE zugeordnet werden können. Fälle, in denen das KEE allein durch den lexikalischen Frame motiviert wird, erscheinen also zunächst ana$\log \mathrm{zu}$ denjenigen, in denen alle KtE einfach durch den lexikalischen Frame motiviert werden (Unterabschnitt 6.2.1). Dass alle KtE eines Konstrukts durch einen lexikalischen Frame motiviert werden können, lässt allerdings nicht den Schluss zu, dass auch das KEE analog motiviert werden kann. Eine Arbeitsteilung zwischen zwei FE ist also nicht in jedem Fall möglich. Einerseits liegt ein möglicher Grund dafür in dem Problem, dass die für das Englische entwickelten Frames in FrameNet 1.7 in dieser Hinsicht nur bedingt auf das Deutsche zu übertragen sind, wenn man beabsichtigt, sowohl dem KtE des KE BEWEGENDES als auch dem KEE jeweils ein eigenes FE zuzuordnen. Andererseits aber gibt dieser Befund Anlass zu der Überlegung, ob die Arbeitsteilung zwischen zwei FE auch für das Deutsche nur möglich ist, wenn es sich bei den entsprechenden LE um ,reflexiv gebrauchte، Verben handelt und dies bei ,echt reflexiven' Verben gerade nicht möglich ist, wie es Buscha (1982: 170-171), Eroms (2000: 307, 411), Zifonun (2003: 17), von Polenz

aus frame-semantischer Sicht aufkommenden Probleme dieses Phänomens gehe ich in Unterabschnitt 6.3.1 ein. 
(2008: 126-129) sowie Hoherz (2017: 304) und die Duden-Grammatik (Duden 2016: 408) nahelegen.

Als erstes Beispiel soll einmal mehr Motion dienen. In den Belegen, die ich bisher zitiert habe (z.B. in Unterabschnitt 6.2.1), wurde den KtE des KE BEWEGENDES stets das FE Motion.THEME zugeordnet. Ein FE, das dem KEE zugeordnet werden könnte, fehlt: Eine Arbeitsteilung zwischen zwei FE kann nicht stattfinden. Es ist also denkbar, dass es sich bei LE wie begeben, bewegen, mäandern, ringeln, rücken, schlängeln, verschieben oder winden in Verbindung mit einem Reflexivum (vgl. die Belege in Unterabschnitt 6.2.1) um ,echt reflexive` Verben handelt.

Anders verhält sich dies für die LE, die dem lexikalischen Frame Cause_motion zugeordnet werden können. Dieser verfügt sowohl über ein FE Cause_motion. THEME als auch über ein FE Cause_motion.AGENT. Zwischen diesen beiden FE kann eine Arbeitsteilung stattfinden. Abweichend von der Praxis für Motion kann das FE Cause_motion.THEmE nun dem Reflexivum als KEE zugeordnet werden, während Cause_motion.AGENT für die Motivierung der KtE des KE BEWEGENDES verantwortlich gemacht werden kann. Die Beispiele in (60)-(62) für die LE schieben (push.v) in (60), drängeln (push.v) in (61) und heben (lift.v) in (62) sollen dies illustrieren.

(60) a. Wieder [cause_motion schiebt] [THEME sich] [Agent die Alte] in den Blick, sie will unsere Großmutter vollständig verdecken. (Beyer, Marcel: Spione, Köln: DuMont 2000, S. 80)

b. Laura ist ganz klein, sogar noch ein paar Zentimeter kleiner als ich, aber [Agent $\mathrm{Sie}$ ] [cause_motion $\mathrm{schiebt]}$ [Tнеме $\mathrm{Sich}$ ] durch die Menge wie ein Türsteher. (Bach, Tamara: Marsmädchen, Hamburg: Verlag Friedrich Oetinger 2003, S. 59)

c. [Agent Er] [cause_motion schob] [THEME sich] ächzend nach vorn auf die Kante seines Sessels, erhob sich aber dann trotz seines mächtigen Bauches überraschend mühelos. (Schulze, Ingo: Neue Leben, Berlin: Berlin Verlag 2005, S. 25)

(61) a. [AGENT $\mathrm{Sie}$ [ [cause_motion drängelt] [THEME $\mathrm{sich}$ ] noch enger an ihn. (Dölling, Beate: Hör auf zu trommeln, Herz, Weinheim: Beltz \& Gelberg 2003, S. 81)

b. [AGENT Katharina] [cause_motion drängelte] [THEME sich] durch die Leute. (Dölling, Beate: Hör auf zu trommeln, Herz, Weinheim: Beltz \& Gelberg 2003, S. 59)

c. [AGENT Sie] [cause_motion drängelt] [Tнеме $\mathrm{Sich}$ ] in den Saal zurück, trifft überall Leute, bleibt aber nicht stehen. (Dölling, Beate: Hör auf zu trommeln, Herz, Weinheim: Beltz \& Gelberg 2003, S. 136) 
a. Und da der Flügel stark genug ist, um den Rumpf zu tragen, [Cause_motion hebt] [Theme $\mathrm{sich}$ [ [Agent das ganze Flugzeug] in die Höhe. (Die Zeit, 05.01.2000, Nr. 2)

b. Dann [Cause_motion hebt] [Theme $\operatorname{sich}$ ] [Agent ihr Kopf] lang in die Stille hinein, ich komme in ihr, Marah krümmt sich weiter vom Boden weg. (Schrott, Raoul: Tristan da Cunha oder die Hälfte der Erde; Hanser Verlag 2003, S. 543)

Die Unterschiede zwischen Frames wie Motion und Cause_motion sind offensichtlich und systematisch: Cause_motion ist Motion in der Kausativ-Relation übergeordnet (vgl. Tabelle 5.16 in Unterabschnitt 5.4.3), was das zusätzliche FE Cause_motion.AGENT erklärt. ${ }^{76}$ In traditionellen Termini, die etwa Ágel (2007) verwendet, handelt es sich bei Cause_motion um einen kausativen Frame, während Moti on ein rezessiver Frame ist. ${ }^{77}$ Kausative Frames sind in FrameNet häufig daran zu erkennen, dass sie den Ausdruck Cause im Namen tragen. Zudem verfügen sie meist (aber nicht immer) ${ }^{78}$ über ihnen in der Kausativ-Relation untergeordnete rezessive Frames. Aus der Reihe der für die reflexive Bewegungskonstruktion belegten unrelatierten Frames besitzen in FrameNet 1.7 etwa Cause_bodily_experience, Cause_to_amalgamate oder Cause_to_move_in_place mit Perception_body, Amalgamation und Moving_in_place rezessive Pendants. Kausative Frames müssen in FrameNet allerdings nicht zwingend in dieser Form gekennzeichnet sein. Neben Cause_motion findet sich unter den belegten relatierten lexikalischen Frames etwa Bringing als kausativer Frame. Die Belege in (63) und (64) zeigen noch einmal

76 Zwar wird die Kausativ-Relation in der FrameNet-Dokumentation von Ruppenhofer et al. (2016: 85), wie für andere FrameNet-bezogene Schriften schon Busse (2012: 190) kritisiert, „,nicht näher definiert, sondern nur an Beispielen erläutert“, besonders in der Darstellung von Petruck et al. (2004), in der sie gemeinsam mit der Inchoativ-Relation eingeführt wird (vgl. schon Unterabschnitt 5.4.3). Allerdings sind beide Relationen, wie hier die systematischen Zusammenhänge um die Kausativ-Relation zeigen, anders als Busse (2012: 190) sie bezeichnet, keineswegs als „,adhoc-Kategorien“ zu verstehen.

77 Der Terminus rezessiv findet sich auch in Duden (2016: 409). Statt rezessiv sind unter anderem auch die Begriffe inchoativ und antikausativ geläufig (vgl. für einen Überblick aus typologischer Perspektive Haspelmath 1987: 8-10). Ersterer ist aufgrund der gleichnamigen Frame-zu-FrameRelation in FrameNet für die vorliegenden Zwecke ungeeignet. Ich folge mit der Dichotomie kausativ und rezessiv deshalb Ágel (2007). Semantisch korreliert Ágel (2017: 344) explizit für Reflexivkonstruktionen die Unterscheidung zwischen rezessiv und kausativ mit den Begriffen endoaktiv und exoaktiv. Über das unterschiedliche Inventar an FE in den jeweiligen Frames (vgl. Tabelle 6.9) lässt sich dieser genuin semantische Unterschied erkennen.

78 Frames, die ein Cause im Namen tragen, aber nicht über untergeordnete rezessive Frames verfügen sind unter den für die reflexive Bewegungskonstruktion belegten etwa Cause_harm oder Cause_to_experience. 
Konstrukte für die LE bringen (bring.v) und schleppen (schlep.v), die Bringing als lexikalischen Frame evozieren.

(63) a. [AgEnt Der Mann, der auf sexuelle Avancen nicht eingeht], [Bringing bringt] [Theme sich] in den Verdacht der Unmännlichkeit. (Schwanitz, Dietrich: Männer, Frankfurt a. M.: Eichborn 2001, S. 208)

b. [Agent Die] [Bringing bringen] [Theme sich] nicht unnötig in Gefahr. (Pressler, Mirjam: Malka Mai, Weinheim Basel: Beltz \& Gelberg 2001, S. 292)

c. [AGENT Die,Herrschaften'] [Bringing bringen] [Theme $\mathrm{sich}$ ] in einer lächerlich-grotesken Flucht in Sicherheit, rennen über die Stufen davon, verschwinden (an dieser Stelle: wandte man. die komischen Abgänge von Zirkusspielern an). (Schlögel, Karl: Petersburg, München Wien: Carl Hanser Verlag 2002, S. 463)

(64) a. [AgENT Ich] [Bringing schleppe] [THeme mich] nach Hause, 2,5 km, schaffe es, die Tür aufzuschließen, die Treppe hoch, und da stehe ich auch schon in meinem Zimmer. (Braun, Marcus: Hochzeitsvorbereitungen, Berlin: Berlin Verlag 2003, S. 114)

b. [AGENT Schwere, sonnenlahme Schildkröten] [Bringing schleppten] [TнEмE sich] über rissigen Uferschlick und versanken in schlammbraunem Wasser. (Düffel, John von: Houwelandt, Köln: DuMont Literatur und Kunst Verlag 2004, S. 142)

c. [AgENT Großvaters Uhrenzeiger] [Bringing schleppten] [Theme sich] von Minute zu Minute, zauderten und zockelten, als seien sie widerwillige Pferde, und Zeigern konnte man mit einer Peitsche nicht kommen! (Koneffke, Jan: Paul Schatz im Uhrenkasten, Köln: DuMont Buchverlag 2000, S. 40)

Dass kausative Frames aber nicht zwangsläufig über die Kausativ-Relation und durch Cause im Namen in FrameNet 1.7 integriert sein müssen, zeigt die Einordnung der in Unterabschnitt 5.4.3 aufgelisteten zum Konstruktions-Frame Motion relatierten lexikalischen Frames in Tabelle 6.9. Dort sind die relatierten lexikalischen Frames nach ihrem Status als rezessiv oder kausativ und den unterschiedlichen Frame-zu-Frame-Relationen geordnet gegenübergestellt. Für die kausativen Frames sind zudem jeweils die beiden FE angegeben, die für die Motivierung der KtE von BEWEGENDES und das KEE in einer Arbeitsteilung infrage kommen. Deutlich zu sehen ist, dass weder die Kausativ-Relation noch die Kennzeichnung Cause im Namen nötig ist, um einen Frame als kausativ zu verstehen. Welche der kausativen Frames in Tabelle 6.9 nun für die einfache Motivierung des KEE der reflexiven 
Bewegungskonstruktion durch lexikalische Frames belegt sind, kann durch einen Vergleich mit Tabelle 6.2 in Unterabschnitt 6.2.1 ermittelt werden.

Tab. 6.9: Kausative und rezessive relatierte lexikalische Frames des Systems der Frame-Nähen von Motion in FrameNet 1.7

\begin{tabular}{|c|c|c|}
\hline Frame-zu-Frame-Relation & Rezessiv & Kausativ (FE) \\
\hline- & Motion & \\
\hline \multirow[t]{5}{*}{ Vererbung $(+1)$} & Fluidic_motion & \\
\hline & Mass_motion & \\
\hline & Motion_directional & \\
\hline & Motion_noise & \\
\hline & Self_motion & \\
\hline \multirow[t]{4}{*}{ Vererbung $(+2)$} & Cotheme & \\
\hline & Fleeing & \\
\hline & Intentional_traversing & \\
\hline & Travel & \\
\hline Vererbung $(-1)$ & Event & \\
\hline Kausativ $(-1)$ & & Cause_motion (AGENT, THEME) \\
\hline \multirow[t]{15}{*}{ Benutzt $(+1)$} & Body_movement & \\
\hline & & Bringing (AgENT, THEME) \\
\hline & Change_direction & \\
\hline & Cotheme & \\
\hline & Departing & \\
\hline & Emanating & \\
\hline & & Evading (EVADER, PURSUER) \\
\hline & Excreting & \\
\hline & & Light_movement (EMITTER, BEAM) \\
\hline & & Operate_vehicle (DRIVER, VeHICLE) \\
\hline & Path_traveled & \\
\hline & & Placing (AgEnt, Theme) \\
\hline & & Redirecting (AgENT, THEME) \\
\hline & & Removing (AgENT, THEME) \\
\hline & Roadways & \\
\hline \multirow[t]{7}{*}{ Benutzt $(+2)$} & & Arranging (AGENT, THEME) \\
\hline & Convoy & \\
\hline & & Disembarking (TRAVELLER, VeHICLE) \\
\hline & & Dressing (WeARER, CLOThING) \\
\hline & & Dunking (Agent, Theme) \\
\hline & Entourage & \\
\hline & Facial_expression & \\
\hline
\end{tabular}


Tab. 6.9 - fortgesetzt

\begin{tabular}{|c|c|c|}
\hline \multirow[t]{6}{*}{ Frame-zu-Frame-Relation } & Rezessiv & Kausativ (FE) \\
\hline & & Installing (AgENT, Component) \\
\hline & & Piracy (Perpetrator, VehicLe) \\
\hline & & Sending (SENDER, Theme) \\
\hline & & Storing (AgENT, ThEME) \\
\hline & & Undressing (WeArer, CLOThing) \\
\hline \multirow[t]{5}{*}{ Benutzt (+3) } & & Carry_goods (DISTRIBUTOR, GOODS) \\
\hline & & Delivery (Deliverer, THEME) \\
\hline & Making_faces & \\
\hline & Sent_items & \\
\hline & Store & \\
\hline \multirow[t]{2}{*}{ Sub-Frame $(+1)$} & Getting_underway & \\
\hline & Halt & \\
\hline Perspektive_auf (-1) & Motion_scenario & \\
\hline \multirow[t]{4}{*}{ Siehe_auch $(+1)$} & & Bringing (AgENT, THEME) \\
\hline & & Operate_vehicle (DRIVER, VEHICLE) \\
\hline & Ride_vehicle & \\
\hline & Self_motion & \\
\hline \multirow[t]{3}{*}{ Siehe_auch $(+2)$} & & Passing (Agent, Theme) \\
\hline & & Sending (SENDER, THEME) \\
\hline & Spatial_contact & \\
\hline \multirow[t]{2}{*}{ Siehe_auch $(+3)$} & & Topic (Communicator, TeXt/Topic) \\
\hline & & Wearing (WeARER, CLOThing) \\
\hline
\end{tabular}

Für die Analyse der drei untersuchten Konstruktionen mit Motion als Konstruktions-Frame ergibt sich nun die Situation, dass das KEE für alle Konstrukte, die einen relatierten lexikalischen Frame evozieren, nur dann als durch ein FE dieses Frames motiviert gelten kann, wenn es sich um einen kausativen Frame handelt. Das Problem äußert sich zunächst auf konstruktikographischer Seite: Da es keine zwei FE gibt, die eine Arbeitsteilung in der Motivierung der KtE von BEWEGENDES und des KEE übernehmen können, kann im Falle eines rezessiven Frames nur eines der beiden Strukturelemente annotiert werden. Wie in den Belegen (5)-(20) in Unterabschnitt 6.2.1 zu sehen, entscheide ich mich dafür, die KtE des KE BEwEGENDES mit FE wie Motion.THEME zu annotieren, während das KEE nicht annotiert wird. Daraus ergibt sich freilich eine inkonsistente Annotationspraxis, da für den Fall eines kausativen Frames, wie in den Belegen in (60)-(62) und (63)-(64) die Arbeitsteilung dahingehend wechselt, dass ein FE wie Cause_motion.THEME oder Bringing.THEME nun für die Motivierung des KEE annotiert wird, während die KtE des KE BewEgendes mit Cause_motion.AGENT oder Bringing.AGENT annotiert werden können. Dies gilt im Übrigen auch für die Fälle unrelatierter lexikalischer Frames, in denen das KEE nun durch das FE Motion.THEME motiviert 
wird (Unterabschnitt 6.3.2). Selbst wenn es sich bei einem unrelatierten lexikalischen Frame um einen rezessiven Frame handeln sollte, kann dieser durch den Konstruktions-Frame ,kausativiert‘ werden (vgl. ähnlich Kunze 1997: 135).

Dieses technische Problem, das sich für den vorliegenden Ansatz vorerst nicht umgehen lässt, ist freilich nur die Vorrede zu einer ganzen Reihe theoretischer Diskussionen, die mit der Untersuchung von Reflexivkonstruktionen einhergehen und die gerade für einen frame-semantischen Ansatz noch weit von einer Klärung entfernt sind. Aus allgemein grammatiktheoretischer Perspektive liegt das Problem darin, dass ,echt reflexive“ Verben, wie Ágel (1997b: 70-71) darlegt (bei ihm: ,mediale“ Verben), valenziell nicht vollständig intransitiv sind, während ,reflexiv gebrauchte‘ Verben nicht vollständig transitiv sind. Während die Annahme eines FE für das Reflexivum am ehesten bei ,reflexiv gebrauchten“ Verben (bei ihm: ,reflexive' Verben) gerechtfertigt scheint, ist diese Rechtfertigung für Verben, die im Kontinuum zwischen Intransitivität und Transitivität näher am intransitiven Pol stehen, schwächer. Dass sie einen kausativen und keinen rezessiven Frame evozieren, lässt sich also schwieriger begründen als bei ,reflexiv gebrauchten“ Verben. Die Problemlage lässt sich auf Grundlage des von Ágel (1997b: 71) postulierten Kontinuums zwischen Intransitivität und Transitivität wie in Abbildung 6.4 dargestellt illustrieren.

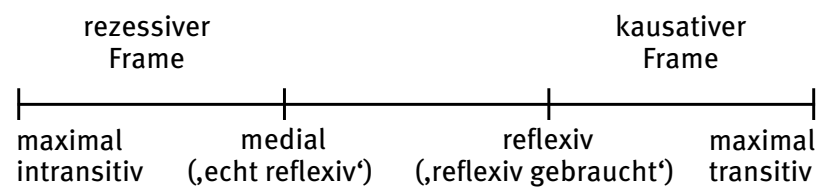

Abb. 6.4: Transitivitäts-Kontinuum (nach Ágel 1997b: 71) und dessen Korrelation zur Kausativität von Frames

Es stellt sich also die Frage, ob all diejenigen Frames, die kein FE zur Motivierung eines Reflexivums zur Verfügung stellen, ,echt reflexive' Verben als LE besitzen, bei denen das Reflexivum aus genau diesem Grund nicht durch ein FE motiviert wird, sondern als zur LE zugehörig angesehen werden muss. Für das Beispiel des lexikalischen Frames Motion würde dies bedeuten, dass die in Unterabschnitt 6.2.1 diskutierten LE nicht nur auf die Verben begeben, bewegen, mäandern, ringeln, rücken, schlängeln, verschieben und winden begrenzt werden können, sondern dass das Reflexivum jeweils mit zur LE gezählt werden muss. Für das Deutsche wären demnach also die LE als sich begeben, sich bewegen, sich mäandern, sich ringeln, sich rücken, sich schlängeln, sich verschieben und sich winden anzusetzen. Dies wäre die frame-semantische Umsetzung des z.B. von Buscha (1982: 
170-171), Eroms (2000: 307, 411) und von Polenz (2008: 127) praktizierten Verfahrens, die Reflexiva bei ,echt reflexiven' Verben zu ebendiesen Verben zugehörig zu betrachten. In FrameNet-Terminologie wären also die eben aufgezählten komplexen LE aus Verb und Reflexivum anzusetzen. ${ }^{79}$

Ein damit zusammenhängendes Problem aus frame-semantischer Perspektive ist schließlich die schon angesprochene Eigenheit, dass die Frames im FrameNet für das Englische entwickelt worden sind und zwar grundsätzlich (vgl. Unterabschnitt 2.1.2), aber eben nicht in jedem Fall auf das Deutsche angewendet werden können. Reflexivkonstruktionen, die über rezessive Frames erfasst werden sollen, stellen einen Phänomenbereich dar, für den sich solche Diskrepanzen ergeben. So ist es eindeutig, dass viele im Englischen nicht-reflexive Verben, die problemlos mit einem rezessiven Frame erfasst werden können, im Deutschen reflexiv sind (vgl. Wagner 1977: 68-69; Oya 2002: 968; Zifonun 2003: 67; Ágel 2017: 343-344; Welke 2019: 435-438; Mortelmans \& Smirnova 2020: 62). Wie mit dem Reflexivum im Deutschen letztlich umzugehen ist, ob die Verben, die als deutsche LE für einen rezessiven Frame infrage kommen, tatsächlich immer auch ,echt reflexive` Verben sind, muss durch zukünftige Studien geklärt werden. Für den Fall, dass sich die Korrelation zwischen, echt reflexiven` Verben und rezessiven Frames sowie ,reflexiv gebrauchten` Verben und kausativen Frames nicht bestätigt, müssen die einschlägigen (für das Englische rezessiven) Frames für das Deutsche angepasst werden. Solange muss es bei der skizzierten Annotationspraxis und den damit verbundenen Annahmen der Motivierung des KEE einer Reflexivkonstruktion bleiben.

\subsubsection{Einfache Motivierung durch Konstruktions-Frame}

Eine einfache Motivierung des KEE der drei untersuchten Konstruktionen liegt immer dann vor, wenn sowohl der Konstruktions-Frame als auch ein zu diesem in keiner Frame-Nähe stehender (also unrelatierter) lexikalischer Frame an der Konstitution eines Konstrukt-Frames beteiligt ist. Anders als bei der einfachen Motivierung des KEE durch den lexikalischen Frame, die mit für das Englische entwickelten rezessiven Frames wie Motion oder Self_motion nicht unmittelbar erklärt werden kann (vgl. Unterabschnitt 6.3.1), steht für den Fall der einfachen Motivierung des KEE durch den Konstruktions-Frame stets ein FE zur Verfügung:

79 Ähnlich geht im Übrigen das elektronische Valenzwörterbuch E-VALBU vor (https://grammis. ids-mannheim.de/verbvalenz, zuletzt abgerufen am 07.09.2021): Dort findet sich für das Verb bewegen ein eigener Eintrag mit dem Lemma bewegen, sich, der also das Reflexivum mit zum Verb zählt. Die anderen hier betrachteten Verben sind dort nicht verzeichnet. 
Motion.THEME. Während rezessive Frames bei der einfachen Motivierung des KEE durch den (relatierten) lexikalischen Frame Anlass zu einer Differenzierung von ,echt reflexiven' und,reflexiv gebrauchten'Verben geben, ist diese Problematik bei unrelatierten lexikalischen Frames einfacher aufzulösen, da die Motivierung des KEE nun durch den Konstruktions-Frame übernommen werden kann:

Aus nicht-kausativen Verben der verschiedensten Art werden kausative Ortswechselverben, die zusätzliche Prädikation [d.h. der Konstruktions-Frame, A.W.] bezieht sich auf eine Lokation des Subjekt-Arguments. (Kunze 1997: 135)

Aus diesem Grund erscheint es plausibel, dass es sich bei all denjenigen LE, die innerhalb einer Konstruktion wie der reflexiven Bewegungskonstruktion einen unrelatierten lexikalischen Frame evozieren, stets um ,reflexiv gebrauchte، Verben handelt, da ihr gemeinsames Auftreten mit einem Reflexivum nicht bereits durch den (relatierten) lexikalischen Frame erklärt werden kann, sondern durch eine Motivierung durch den Konstruktions-Frame lizenziert wird.

Um den Unterschied zwischen der einfachen Motivierung des KEE durch den lexikalischen Frame und den Konstruktions-Frame zu verdeutlichen, seien ein Beleg für den lexikalischen Frame Motion als relatierten lexikalischen Frame in (65) und ein Beleg für den Konstruktions-Frame Motion mit dem unrelatierten lexikalischen Frame Ingestion in (66) miteinander verglichen.

(65) Besser wird es nicht, [Theme ich] [Motion begebe] mich sofort [GoAL in mein Bett]. (Braun, Marcus: Hochzeitsvorbereitungen, Berlin: Berlin Verlag 2003, S. 71)

(66) Auch wenn ich als Kind die Inhalte der Angst nicht kapierte, [Ingestion fraß] [sich Theme] [Ingestor das Gefühl der Angst] [Ingestibles in den Kopf Goal]. (Müller, Herta: Der König verneigt sich und tötet, München: Carl Hanser Verlag 2003, S. 161)

In (65) motiviert das FE Motion.THeme das KtE des KE Bewegendes. Für das KEE steht, wie in 6.3.1 argumentiert, kein FE zur Verfügung. In (66) hingegen ist eine Arbeitsteilung zwischen einem FE des lexikalischen Frames und dem FE Motion.Theme des Konstruktions-Frames möglich. Der lexikalische Frame Ingestion kann nun das KtE des KE BEwEGENDES motivieren, sodass das FE Motion.THEME für das KEE zur Verfügung steht. Diese unterschiedliche Annotation des KEE muss aus praktischen Gründen in Kauf genommen werden, bringt

80 Dass die lexikalische Bedeutung des Verbs, anders als Kunze argumentiert, allerdings erhalten bleibt und die ,zusätzliche Bedeutung lediglich in Form des Konstrukt-Frames und der Konstruktbedeutung entsteht, sollte mittlerweile deutlich geworden sein. 
aber einen Vorteil mit sich: An der einfachen Motivierung des KEE durch den Konstruktions-Frame ist sofort erkennbar, dass der Konstrukt-Frame ein Blend aus lexikalischem Frame und Konstruktions-Frame ist und somit beide FrameTypen im Spiel sind, also eine Frame-Anpassung stattfindet. Zudem lässt sich in vielen Fällen aufgrund der Motivierung des KEE durch das FE Motion.THEME erst das Auftreten des Reflexivums mit LE erklären, die, wie fressen in (66), ein solches eigentlich nicht erwarten lassen. Mit der semantischen Motivierung des KEE geht damit die syntaktische Lizenzierung des Reflexivums einher (vgl. auch Unterabschnitt 6.1.2).

Analog zu der Verteilung lexikalischer Frames, die von einer einfachen Motivierung des KE WEG durch den Konstruktions-Frame betroffen sind (Unterabschnitt 6.2.2, Tabelle 6.3) lassen sich auch diejenigen unrelatierten lexikalischen Frames ermitteln, zu denen im Konstrukt-Frame das FE Motion.THEME hinzutritt, das das KEE einfach motiviert. Wie Tabelle 6.10 zeigt, betrifft dies insgesamt 104 Konstrukte und 21 verschiedene lexikalische Frames.

Tab. 6.10: Einfache Motivierung des KEE der reflexiven Bewegungskonstruktion durch den Konstruktions-Frame nach unrelatierten lexikalischen Frames

\begin{tabular}{lrr}
\hline Lexikalischer Frame & Konstrukte & Anteil \\
\hline Daring & 18 & $17,31 \%$ \\
Seeking & 14 & $13,46 \%$ \\
Work & 13 & $12,50 \%$ \\
Hostile_encounter & 11 & $10,58 \%$ \\
Ingestion & 10 & $9,62 \%$ \\
Scouring & 7 & $6,73 \%$ \\
Manipulation & 4 & $3,85 \%$ \\
Chatting & 3 & $2,88 \%$ \\
Desiring & 3 & $2,88 \%$ \\
Impact & 3 & $2,88 \%$ \\
Make_noise & 3 & $2,88 \%$ \\
Others_situation_as_stimulus & 3 & $2,88 \%$ \\
Reshaping & 3 & $2,88 \%$ \\
Progression & 2 & $1,92 \%$ \\
Cause_bodily_experience & 1 & $0,96 \%$ \\
Cause_harm & 1 & $0,96 \%$ \\
Dead_or_alive & 1 & $0,96 \%$ \\
Hunting & 1 & $0,96 \%$ \\
Perception_experience & 1 & $0,96 \%$ \\
Prevarication & 1 & $0,96 \%$ \\
Shopping & 1 & $0,96 \%$ \\
\hline Gesamt & 104 & $100,00 \%$ \\
\hline
\end{tabular}


Als frequentester lexikalischer Frame, zu dem eine einfache Motivierung des KEE durch den Konstruktions-Frame tritt, ergibt sich Daring, der bereits hinsichtlich der einfachen Motivierung des KtE WEG durch den Konstruktions-Frame (vgl. Tabelle 6.3 in Unterabschnitt 6.2.2) den für die reflexive Bewegungskonstruktion frequentesten lexikalischen Frame darstellt. Die Belege in (67) und (68) sind für die LE (ge)trauen und wagen (beide dare.v) Beispiele für den Fall, in dem das KtE des KE BEWEGENDES jeweils durch das FE Daring.AGENT des lexikalischen Frames motiviert wird und das KEE einzeln durch das FE Motion.THEME des KonstruktionsFrames.

a. Aber früher, als wir noch klein waren, [Daring traute] [sich ${ }^{\text {THEME}}$ ] niemand an uns heran, auch nicht in Mückes Gegend, denn wir standen unter dem Schutz seines Bruders, und dessen Rache würde fürchterlich sein. (Goosen, Frank: Liegen lernen, Frankfurt am Main: Eichborn AG 2000, S. 88)

b. Er [Daring traute] [sich ${ }^{\text {THEME }] ~ n i c h t ~ m e h r ~ a u s ~ d e m ~ H a u s, ~ h o ̈ r t e ~ i m m e r ~}$ wieder Stimmen, fühlte sich bedroht und sah im Spiegel jemanden, den er nicht kannte. (Die Zeit, 09.03.2000, Nr. 11)

c. Er machte sich Vorwürfe und [Daring getraute] [sich ${ }^{\text {THEME}] ~ a u s ~ s c h l e c h-~}$ tem Gewissen nicht einmal mehr zu seinen Kindern. (Wondratschek, Wolf: Mozarts Friseur, München, Wien: Carl Hanser Verlag 2002, S. 79)

(68) a. Trotzdem [Daring wagen] [sich ${ }^{\text {THEME}}$ ] laufend neue B2B-Firmen an die Börse. (Die Zeit, 03.02.2000, Nr. 6)

b. Er meidet diese Gegend, [Daring wagt] [sich ${ }^{\text {THEME}}$ ] nicht auf den Hügel herauf, fürchtet noch den entferntesten Blickkontakt mit einem Mitglied seiner Familie. (Beyer, Marcel: Spione, Köln: DuMont 2000, S. 126)

c. Seit es nicht mehr allzu riskant ist, [Daring wagen] [sich THEME] auch andere aus der jüngeren CDU-Generation gegen den Altkanzler vor. (Die Zeit, 03.02.2000, Nr. 6)

d. Mehr und mehr französische Politiker [Daring wagen] [sich THEмE] in Unterhaltungssendungen. (Die Zeit, 27.04.2000, Nr. 18)

e. Das Kleeblatt [Daring wagt] [sich ${ }^{\text {THEME}}$ ] nach draußen. (Braun, Marcus: Hochzeitsvorbereitungen, Berlin: Berlin Verlag 2003, S. 19)

Bevor ich auf den lexikalischen Frame Seeking eingehe, der an zweiter Stelle nach Daring liegt und für den eine Besonderheit in der semantischen Motivierung des KEE zu beobachten ist, sei zunächst ein Blick auf den in der Frequenz an dritter Stelle stehenden Frame Work geworfen. Die Arbeitsteilung bei der Motivierung der KtE des KE BEWEGENDES und des KEE erfolgt gemeinsam mit dem FE Work.AGENT, 
das nun die KtE des KE BewEGEndes motiviert. Die Belege in (69) sind Beispiele dafür.

(69) a. Die Ermittler [work arbeiteten] [sich ${ }^{\text {THEME}}$ ] an den großen Zampano heran. (Die Zeit, 27.04.2000, Nr. 18)

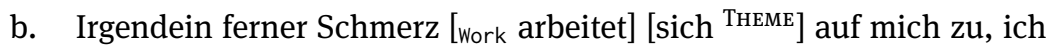
sehe ihn kommen. (Genazino, Wilhelm: Die Liebesblödigkeit, München, Wien: Carl Hanser Verlag 2005, S. 108)

c. „Manchmal [work arbeiten] [sich ${ }^{\text {THEME] }}$ aus der teigigen, formlosen Volksmasse irgendwelche besonderen, starken, sehr arbeitsfähigen Menschen nach oben. (Schlögel, Karl: Petersburg, München Wien: Carl Hanser Verlag 2002, S. 292)

d. Sie [work arbeitete] [sich ${ }^{\text {THEME}] ~ d u r c h ~ s c h u l t e r h o h e s ~ D i c k i c h t ; ~ d i e ~ M a ̈ n-~}$ ner waren angeseilt, da sich unter dem Moosbewuchs tiefe Felsspalten verbargen; im stellenweise dichten Nebel hätte man sich ohne Kompaß leicht verirrt. (Schrott, Raoul: Tristan da Cunha oder die Hälfte der Erde; Hanser Verlag 2003, S. 31)

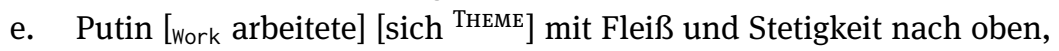
nicht mit Brillanz. (Die Zeit, 23.03.2000, Nr. 13)

f. Sein Herz [work arbeitete] [sich ${ }^{\text {THEME}] ~ v o r a n, ~ i m m e r ~ h o ̈ h e r, ~ i m m e r ~ d i c h-~}$ ter unter die Haut. (Schulze, Ingo: Neue Leben, Berlin: Berlin Verlag 2005, S. 751)

g. Man begann bei den Gesetzen der Körperwelt, [work arbeitete] [sich Tнеме] empor zur Psychologie, zu den Gesetzen des Empfindens und Denkens, Ausflüge in die Schöne Literatur waren vorgesehen, sie ermöglichten den Übergang zum Feinseelischen und zu den letzten Fragen, Gott und Unsterblichkeit. (Safranski, Rüdiger: Friedrich Schiller, München Wien: Carl Hanser 2004, S. 46)

Der lexikalische Frame Hostile_encounter, der ebenfalls bereits bei der einfachen Motivierung der KtE des KE WEG durch den Konstruktions-Frame einer der frequentesten ist, tritt in einer ähnlichen Rolle auch bei jener Motivierung des KEE auf. Anders als bei den lexikalischen Frames Daring und Work wird in allen Konstrukten von Hostile_encounter, in denen das KtE des KE WEG bereits einfach durch den Konstruktions-Frame motiviert ist, auch das KEE einfach durch den Konstruktions-Frame motiviert. Für die Motivierung der KtE des KE BEWEGENDES ist nun das FE Hostile_encounter.SIDE_1 zuständig. Einige Belege sind für die LE kämpfen und kloppen (beide fight.v) sowie ringen (struggle.v) sind in (70)-(72) zusammengestellt. 
(70) a. Ich [Hostile_encounter kämpfte] [mich THEME] durch dieses Unterholz, hielt mich an den Stämmen, Asten und Wurzeln fest, weil ich hinauf zum Gipfel wollte, der wie jeden Tag in den Wolken lag; wie dick die Schicht war, ließ sich nicht sagen - vielleicht schien oben die Sonne. (Schrott, Raoul: Tristan da Cunha oder die Hälfte der Erde; Hanser Verlag 2003, S. 161)

b. Das von Uganda unterstützte MLC BEMBAs, mittlerweile eine der größten Rebellengruppen, kontrolliert weite Gebiete im Osten und Norden des Kongo und [Hostile_encounter kämpft] [sich ${ }^{\text {THEME}] ~ i n ~ R i c h-~}$ tung der Hauptstadt Kinshasa vor. (Archiv der Gegenwart, 2001 [2000])

(71) Im Video zu Berlin liebt dich [Hostile_encounter kloppen] [sich ${ }^{\text {THEmE}] ~ S u r r o-~}$ gat in bester Tatort-Manier durch die Großstadt und parodieren nebenbei noch den Beastie-Boys-Clip-Klassiker Sabotage: Patrick unverschämt grinsend im Fitness-Studio, Mai-Linh schwer bewaffnet und gefährlich als Martial Artist, Tilo als smoother Supercop. (Die Zeit, 06.04.2000, Nr. 15)

(72) Maria [Hostile_encounter ringt] [sich THEмE] in Wirklichkeit zu einer inneren Freiheit durch, die es ihr erlaubt, Verantwortung für ihr Leben zu übernehmen, das ungerechte Urteil und den Tod als Sühne hinzunehmen und in einen Akt der Freiheit zu verwandeln. (Safranski, Rüdiger: Friedrich Schiller, München Wien: Carl Hanser 2004, S. 478)

Die Motivierung der einzelnen Strukturelemente durch den Konstruktions-Frame ist also grundsätzlich unabhängig voneinander und hängt vielmehr von dem lexikalischen Frame und der Präposition ab, die den Kopf des KtE des KE WEG bildet. Wird das KEE durch den Konstruktions-Frame einfach motiviert, bedeutet dies nicht automatisch, dass auch andere Strukturelemente, insbesondere die KtE des KE WEG, ebenso einfach durch den Konstruktions-Frame motiviert werden müssen (dazu Unterabschnitt 7.4.1). Umgekehrt gilt nicht, dass eine einfache Motivierung von KtE des KE WEG auch mit einer einfachen Motivierung des KEE einhergehen muss, was am deutlichsten an den teils unterschiedlichen Frequenzen von Konstrukten für die lexikalischen Frames in den Tabellen 6.3 und $6.10 \mathrm{zu}$ erkennen ist. Am Beispiel von Seeking seien diese Zusammenhänge aus Sicht der einfachen Motivierung des KEE illustriert.

Für den lexikalischen Frame Seeking sind zwei Kombinationen der Motivierung der Konstrukte belegt. Während das KEE stets einfach durch den Konstruktions-Frame motiviert wird, können die KtE des KE WEG auf zwei Arten motiviert werden. Die erste Variante ist eine einfache Motivierung durch den KonstruktionsFrame, wie dies in den Konstrukten in (73) zu sehen ist. 
(73) a. Wir [seeking tasteten] [uns ${ }^{\text {THEME }] ~ l a n g s a m ~[d u r c h ~ d a s ~ D u n k e l, ~ i n ~ d e m ~}$ es kein Ziel, keine Richtung mehr gab, nur den Gestank von totem Wasser und das schwache Tröpfeln der Rinnsale, unterbrochen vom platschenden Schritt unserer unsichtbaren Füße und dem Stöhnen unseres beklommenen Atems ${ }^{\text {PATH }}$. (Düffel, John von: Vom Wasser, München: dtv 2006, S. 39)

b. Die Frau nimmt nicht den Fahrstuhl, sondern [seeking tastet] [sich ${ }_{\text {THEME] [über die Treppen }}{ }^{\mathrm{PATH}}$ ] hinauf in den sechsten Stock, vorbei an den schlafenden Bettlern, die der heilige Martin vergaß. (Venske, Regula: Marthes Vision, Frankfurt am Main: Eichborn Verlag 2006, S. 184)

In der zweiten Variante werden die KtE des KE WEG doppelt motiviert, einerseits durch das FE Motion.GoAL des Konstruktions-Frames, andererseits durch das FE Seeking.SougHT_ENTITY des lexikalischen Frames, wie in den Konstrukten in (74) zu sehen.

(74) a. Die jetzt 30-köpfige Truppe der Poststaatsfarmer [seeking tappt] [sich THEME] nach Hörensagen [Sought_ENTITy an EU-Vorschriften GoAL] heran, entsorgt die Gülle bodenschonend, gibt den Schweinen mehr Kräuter als Hormone. (Die Zeit, 16.03.2000, Nr. 12)

b. Dabei [seeking tastet] [sich ${ }^{\text {THEME}}$ ] die Elektronik [Sought_Entity an jenen Punkt heran, an dem die Motordrehzahl zu sinken beginnt ${ }^{\text {GoAL }}$. (Die Zeit, 06.04.2000, Nr. 15)

c. Schiller [seeking tastet] [sich ${ }^{\text {THEME}}$ ] [Sought_entity an den Urschmerz des Bewußtseins ${ }^{\text {GoAL }}$ ] heran, an jenen Augenblick, da das erwachende Bewußtsein die unmittelbare Leichtigkeit des Seins, die traumwandlerische Sicherheit der natürlichen Lebensvollzüge, die Unbefangenheit verliert. (Safranski, Rüdiger: Friedrich Schiller, München Wien: Carl Hanser 2004, S. 419)

Neben dem lexikalischen Frame spielt freilich die Präposition, die den Kopf des KtE des KE WEg bildet, eine Rolle. In Konstrukten wie denen in (73) mit den Präpositionen durch und über ist lediglich eine einfache Motivierung durch FE wie Motion.PATH denkbar. Handelt es sich jedoch um die Präposition an, wie in den Konstrukten in (74), erscheint es plausibel, dass das KtE neben dem FE Motion.GoAL zusätzlich durch das FE Seeking.SouGHT_ENTITY motiviert wird. Darüber hinaus spielt freilich die Frage, ob das durch das KtE des KE WEG bezeichnete Referenzobjekt, wie in (73), als eine ,SoUGHT_ENTITY' interpretiert werden kann oder, wie in (73), nicht. Entscheidend ist hier die Konstruktbedeutung, die aus dem entsprechenden Konstrukt-Frame hervorgehen soll. 
Die Kombinationen von Motivierungen unterschiedlicher Strukturelemente eines Konstrukts haben das Potenzial, Konstrukt-Frames nach eigenen Mustern, ähnlich den Valenzmustern in FrameNet (Unterabschnitt 2.1.2), zu bilden. Für die reflexive Bewegungskonstruktion und analog für die reflexive Partikelverbkonstruktion und die reflexive Weg-Konstruktion leuchtet es ein, dass diese Muster primär von Präferenzen für lexikalische Frames (Unterabschnitt 5.3.3) und sekundär von den Präpositionen als Köpfe der KtE des KE WEG sowie der Konstruktbedeutung abhängen. Solche Muster zu untersuchen dient der Differenzierung von Koerzionseffekten in einzelnen Konstrukten und ist damit Voraussetzung für die Messung des Koerzionspotenzials (Unterabschnitt 7.4.1).

\subsubsection{Doppelte Motivierung des KEE}

Analog zu der doppelten Motivierung der KtE des KE WEG (Unterabschnitt 6.2.3) kann auch das KEE der reflexiven Bewegungskonstruktion doppelt motiviert werden. Die hierbei involvierte konzeptuelle Integration von lexikalischem Frame und Konstruktions-Frame hin zu einem Konstrukt-Frame stellt sich bezogen auf die beiden FE, die das KEE motivieren, genauso dar wie bei der doppelten Motivierung eines KtE: Die beiden FE werden fusioniert und sind als Gegenstücke in den Inputs verbunden (vgl. Abbildung 6.5).

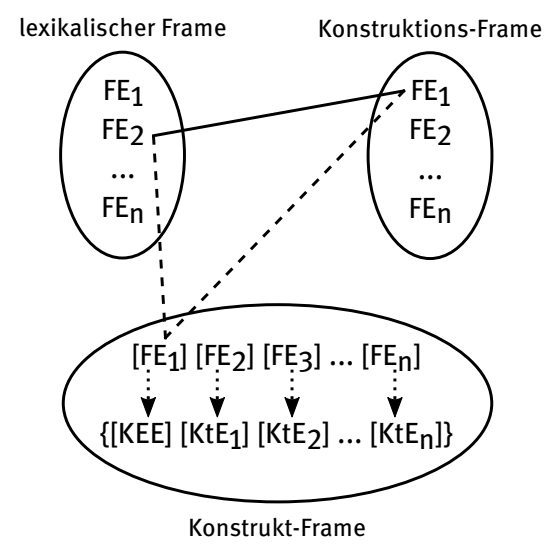

Abb. 6.5: Doppelte Motivierung eines KEE als konzeptuelle Integration

Damit ein KEE doppelt motiviert werden kann, muss dieselbe Voraussetzung gegeben sein wie für dessen einfache Motivierung durch den lexikalischen Frame (Unterabschnitt 6.3.1): Damit der lexikalische Frame überhaupt ein FE zur Motivie- 
rung beitragen kann, muss es sich um einen kausativen lexikalischen Frame handeln, nur dass dies jetzt für unrelatierte lexikalische Frames gilt. Bei rezessiven unrelatierten lexikalischen Frames wie denen, die ich in Unterabschnitt 6.3.2 diskutiert habe, liegt zwangsläufig eine einfache Motivierung durch den KonstruktionsFrame vor. Allerdings muss, selbst wenn es sich um einen kausativen Frame handelt, die Motivierung des KEE nicht in jedem Fall doppelt ausfallen. Wie für die einfache Motivierung des KEE ist jedoch auch bei einer doppelten Motivierung davon auszugehen, dass es sich bei den LE, die die entsprechenden lexikalischen Frames evozieren, um ,reflexiv gebrauchte‘ Verben handelt.

Für insgesamt 74 Konstrukte der reflexiven Bewegungskonstruktion ist eine doppelte Motivierung des KEE belegt. Tabelle 6.11 listet sie nach lexikalischen Frames geordnet auf.

Tab. 6.11: Doppelte Motivierung des KEE der reflexiven Bewegungskonstruktion nach lexikalischen Frames

\begin{tabular}{llrr}
\hline Lexikalischer Frame & FE & Konstrukte & Anteil \\
\hline Cause_to_amalgamate & PART_1 & 30 & $40,54 \%$ \\
Manipulation & ENTITY & 16 & $21,62 \%$ \\
Cause_to_experience & EXPERIENCER & 11 & $14,86 \%$ \\
Rescuing & PATIENT & 5 & $6,76 \%$ \\
Emptying & THEME & 3 & $4,05 \%$ \\
Attaching & ITEM & 2 & $2,70 \%$ \\
Filling & THEME & 2 & $2,70 \%$ \\
Smuggling & GOODS & 2 & $2,70 \%$ \\
Assistance & HELPER & 1 & $1,35 \%$ \\
Burying & THEME & 1 & $1,35 \%$ \\
Grinding & PATIENT & 1 & $1,35 \%$ \\
\hline Gesamt & & 74 & $100,00 \%$ \\
\hline
\end{tabular}

Die beiden frequentesten lexikalischen Frames sind Cause_to_amalgamate und Manipulation. Zunächst zu Cause_to_amalgamate. Neben Motion.THEME wird das KEE in einschlägigen Konstrukten zusätzlich durch das FE Cause_to_amalgamate.PART_1 motiviert, wie an den Belegen in (75)-(77) mit den LE mengen und mischen (beide mix.v) sowie (ein)fädeln (etwa: merge.v) zu sehen ist.

(75) a. Es war verteufelt, Herr Joseph Schatz [Cause_to_amalgamate mischte] [PART_1 sich ${ }^{\text {THEME}}$ ] in seine Aufmerksamkeit. (Koneffke, Jan: Paul Schatz im Uhrenkasten, Köln: DuMont Buchverlag 2000, S. 221) 
b. Selbst Magne Furuholmen von der leicht verdaulichen Popband A-ha [Cause_to_amalgamate mischt] [PART_1 sich $^{\text {THEME }}$ ] unter die Runde der exzentrischen Glasperlenspieler, die an ihrer Version von ,arctic ambient“ weben. (Die Zeit, 20.04.2000, Nr. 17)

c. Unverständliches Genuschel [Cause_to_amalgamate mischte] [PART_1 sich THEME] zwischen einzelne Schluchzer, es war unmöglich zu sagen, ob das Dienstmädchen aus Rührung, Empörung oder Eifersucht weinte. (Düffel, John von: Vom Wasser, München: dtv 2006, S. 206)

(76) Lichtgarben und Wolkenschatten [Cause_to_amalgamate mengen] [PART_1 sich THEME] in den Strom, der in seiner Meeresgrüne schäumend aufblitzt. (Düffel, John von: Vom Wasser, München: dtv 2006, S. 69)

(77) a. Ein Lied singt sie zusammen mit Armand, [Cause_to_amalgamate fädelt] [PART_1 sich ${ }^{\text {THEME}] ~ i n ~ s e i n e ~ T o ̈ n e, ~ u n d ~ e r ~ s p i e l t ~ s i e ~ a n, ~ f a ̈ l l t ~ v o r ~ i h r ~ a u f ~}$ die Knie, kann ihr so unter den Rock gucken. (Dölling, Beate: Hör auf zu trommeln, Herz, Weinheim: Beltz \& Gelberg 2003, S. 205)

b. Ich [Cause_to_amalgamate fädelte] [PART_1 mich ${ }^{\text {THEME}] ~ i n ~ d e n ~ V e r k e h r ~ e i n . ~}$ (Schulze, Ingo: Neue Leben, Berlin: Berlin Verlag 2005, S. 584)

Bevor ich den nach Cause_to_amalgamate zweitfrequentesten Frame Manipulation betrachte, für den hinsichtlich der Motivierung des KEE ähnlich wie bei Seeking (Unterabschnitt 6.3.2) eine Besonderheit vorliegt, sei zunächst ein Blick auf den lexikalischen Frame Cause_to_experience geworfen, der an dritter Stelle steht. In den Konstrukt-Frames bringt er zur Motivierung des KEE das FE Cause_to_experience.EXPERIENCER ein. Die zum Teil bereits mehrfach zitierten Belege für die LE quälen (torment.v) in (78) sind Beispiele dafür.

(78) a. Sie [Cause_to_experience quälten] [EXPERIENCER sich ${ }^{\text {THEME}] ~ v i e l l e i c h t ~ a u c h ~}$ aus Tischen, wenn man voller Zweifel dagegen stieß? (Kuckart, Judith: Lenas Liebe, Köln: DuMont Literatur und Kunst Verlag 2002, S. 123)

b. „Ich sitze hier von morgens bis abends am Schreibtisch und [Cause_to_ experience quäle] [ExPERIEncer mich ${ }^{\text {THEME}}$ ] durch eine Akte nach der anderen. (Düffel, John von: Houwelandt, Köln: DuMont Literatur und Kunst Verlag 2004, S. 137)

c. Vom sonnigen unteren Centovalli aus steigt man über fette Feuersalamander durch Esskastanienwälder bergan, schwitzt, dampft, springt nackt in einen Bergbach, [Cause_to_experience quält] [EXPERIENCER sich THEME] über verrutschte Pfade an Ziegenvolk vorbei ins Baumlose, zieht einen Pullover an, wirft den ersten Schneeball, und bei 1000 Metern über null versinkt man bis zur Hüfte im Schnee. (Die Zeit, 30.03.2000, Nr. 14) 
d. Lukian [Cause_to_experience quält] [ExPERIENCER sich THEME] $\mathrm{zu}$ einem Lächeln. (Krausser, Helmut: Eros, Köln: DuMont 2006, S. 146)

Schließlich seien in (79) einige ebenfalls bereits zitierte Belege für den lexikalischen Frame Rescuing illustriert, aus dem das FE Rescuing.PATIENT in die doppelte Motivierung des KEE eingeht. Er ist für die LE retten (rescue.v) belegt.

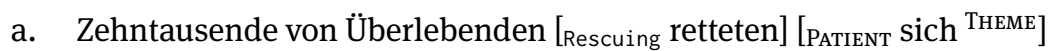
auf Hügel, Bäume und Hausdächer, wo sie ohne Trinkwasser und Nahrungsmittel teilweise tagelang ausharrten, bis sie von Hubschraubern gerettet und in Sammellager geflogen wurden. (Archiv der Gegenwart, 2001 [2000])

b. Mommsen [Rescuing rettete] [Patient sich $^{\text {THEmE}}$ ] aus der Welt der Tatsachen in die der Gefühle: (de Bruyn, Günter: Preußens Luise, Siedler 2001, S. 87)

c. Ich weiß, daß sie mir alles aus dem Gesicht ablesen kann, will es nicht dazu kommen lassen, werde immer linkischer und [Rescuing rette] [PATIENT mich ${ }^{\text {THEME}}$ ] in die Einsilbigkeit. (Schrott, Raoul: Tristan da Cunha oder die Hälfte der Erde; Hanser Verlag 2003, S. 513)

Wie bereits angedeutet, liegt für den Frame Manipulation, der in Tabelle 6.11 nach mit Cause_to_amalgamate der zweitfrequenteste ist, eine Besonderheit hinsichtlich der Motivierung des KEE in Kombination mit derjenigen der KtE des KE WEG vor. Dies ist schon daran zu erkennen, dass dieser Frame sowohl in Tabelle 6.10 in Unterabschnitt 6.3.2 für die einfache Motivierung des KEE durch den Konstruktions-Frame erscheint, als auch in Tabelle 6.11 für die doppelte Motivierung des KEE. Um die Unterschiede zu erkennen, ist die gleichzeitige Betrachtung der Motivierung der KtE des KE WEg von Nutzen. Die Belege in (80) sind daher zunächst Beispiele für die einfache Motivierung des KEE durch den KonstruktionsFrame, wobei ich die Annotation für die KtE des KE WEG ebenfalls darstelle. Als LE ist hier krallen (claw.v) belegt.

(80) a. Er watete mehrere Meter in eisigem Wasser und kroch eine Treppe hoch, [Manipulation krallte] [sich ${ }^{\text {THEME}}$ ] mit seinen Fingern [ENTITY in rieselnden Kalk GoAL]. (Koneffke, Jan: Paul Schatz im Uhrenkasten, Köln: DuMont Buchverlag 2000, S. 217)

b. Einmal, als ich später mit ihnen auf der Flucht war vor dem ebenfalls fliehenden deutschen Heer, lag da ein verwundeter Deutscher und [Manipulation krallte] [sich ${ }^{\text {THEME}}$ ] vor Schmerz [ENTITy in die Erde ${ }^{\text {GoAL}}$ ]. (Hahn, Ulla: Unscharfe Bilder, München: Deutsche Verlags-Anstalt 2003, S. 222) 
Wie an den Belegen in (80) $\mathrm{zu}$ erkennen ist, sind die Referenzobjekte der KtE des KE WEg stets im Sinne einer ,ENTITY‘ des lexikalischen Frames Manipulation zu interpretieren, die als „[t]he ENTITy being manipulated“ (FrameNet 1.7, Manipulation) definiert ist. Hinzu kommt eine Motivierung dieser KtE durch den Konstruktions-Frame, sodass eine doppelte Motivierung entsteht.

Anders verhält sich diese Motivierung bei den Konstrukten in (81) und LE wie quetschen oder zwängen (beide squeeze.v). Hier ist nun nicht mehr das Referenzobjekt eines KtE des KE WEG als manipulierte ,ENTITY‘ zu verstehen, sondern dasjenige des KEE. Es entsteht eine doppelte Motivierung des KEE, während die KtE des KE WEG einzeln motiviert werden.

a. Die Babylonier nickten und [Manipulation quetschten] [ENTITY sich $^{\text {THEME] }}$ ungelenk [durch die Glastür ${ }^{\mathrm{PATH}}$ ] auf die Steinstraße. (Kopetzky, Steffen: Grand Tour, Frankfurt am Main: Eichborn 2002, S. 283)

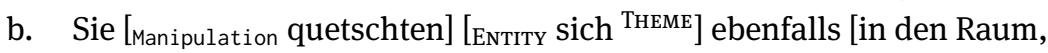
der nicht viel größer als das Ehebett war, das in ihm stand ${ }^{\text {GoAL }}$. (Widmer, Urs: Das Buch des Vaters, Zürich: Diogenes 2004, S. 142)

c. Dann ließ er Bechthold stehen, [Manipulation zwängte] [EnтітY sich ${ }^{\text {THEME] }}$ [durch die Gruppe wahnsinnig Gewordener ${ }^{\text {GoAL }}$ ], kam auf den Würger zu, gab ihm fröhlich die Hand und sagte: (Kopetzky, Steffen: Grand Tour, Frankfurt am Main: Eichborn 2002, S. 725)

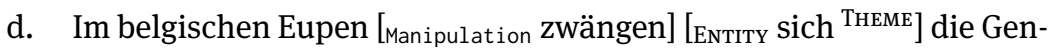
darmen der Rijkswacht mit ihren Computern und Funkanlagen [in die düsteren Kammern einer Kaserne anno $1900^{\mathrm{GOAL}}$ ], klaglos übrigens, weil wenigstens technisch gut versorgt; im Hof stehen Streifenwagen, aber sonst sieht alles aus wie damals, als hier Pferde wieherten. (Die Zeit, 20.04.2000, Nr. 17)

Die Frage, ob das KEE doppelt oder einzeln durch den Konstruktions-Frame motiviert wird, lässt sich erneut zwar anhand der lexikalischen Frames beantworten, die in die Konstrukt-Frames eingehen, die Belege in (80) und (81) zeigen allerdings, dass die lexikalischen Bedeutungen unterschiedlicher LE desselben lexikalischen Frames auch hier Feindifferenzierungen erforderlich machen.

Meine in Unterabschnitt 6.2.3 für die doppelte Motivierung des KE WEG geäußerte Kritik am Semantic Coherence Principle von Goldberg (1995: 50-52) gilt ebenso für die Motivierung des KEE. Die wenigsten FE lexikalischer Frames, die gemeinsam mit dem FE Motion.THEME das KEE motivieren, lassen sich als spezifischere Instanzen dieses FE betrachten. In Tabelle 6.12 sind die drei lexikalischen Frames samt FE zusammengefasst, für die eine Kohärenz mit dem FE Motion.THEME des Konstruktions-Frames noch plausibel erscheint. 
Tab. 6.12: Mögliche Parallelen zwischen dem FE Motion.THEME des Konstruktions-Frames und FE lexikalischer Frames der reflexiven Bewegungskonstruktion

Motion.THEME

Burying.THEME

Emptying.THEME

Filling.THEME

\subsection{Sonderfälle}

In den letzten beiden Abschnitten 6.2 und 6.3 habe ich jeweils einzelne KtE sowie das KEE der reflexiven Bewegungskonstruktion in den Blick genommen und die semantische Motivierung dieser Strukturelemente durch FE des lexikalischen Frames, des Konstruktions-Frames oder beider zugleich untersucht. Diese Untersuchungen sind für eine konstruktikographische Analyse äußerst relevant, da sie Aufschluss über einige zentrale Eigenschaften der Konstruktion geben und Ausgangspunkt von deren Analyse sind: ihr Koerzionspotenzial (Abschnitt 5.5), ihre Produktivität (Abschnitt 5.6) und ihre emergente Struktur (Abschnitt 5.7). Dies sind nicht zufällig drei semantische Parameter, die auch Eingang in einen Konstruktionseintrag finden müssen (Unterabschnitt 7.1.3) und für deren Messung die Analyse der Strukturparallelen eine Voraussetzung ist. Auf die dahingehende Verwertbarkeit dieser Analysen werde ich deshalb insbesondere in Kapitel 7 (besonders in den Abschnitten 7.4 bis 7.6) zurückkommen. Bisher noch nicht betrachtet wurden allerdings einige in den Daten auftretende Fälle, die teilweise im Zusammenhang mit der in den Abschnitten 6.2 und 6.3 diskutierten Klassifikation von Parallelen zwischen den Strukturelementen der Konstrukte einer Konstruktion und den FE von lexikalischen Frames und dem Konstruktions-Frame stehen. Zudem fehlen noch Erkenntnisse über die reflexive Partikelverbkonstruktion, welche sich bei der Analyse der Strukturparallelen von der reflexiven Bewegungskonstruktion in einigen Aspekten unterscheidet.

In diesem Abschnitt geht es um solche Sonderfälle. Zunächst untersuche ich den für die reflexive Bewegungskonstruktion öfter belegten Fall, dass gleich mehrere FE des Konstruktions-Frames, die die KtE des KE WEG motivieren, instanziiert werden: Motion.SourCE, Motion.PATH, Motion.GoAL und Motion.DiReCTION können nicht nur abwechselnd über verschiedene Konstrukte hinweg auftreten, sondern auch gemeinsam in einem Konstrukt. Ich spreche in diesem Fall von einer mehrfachen Instanziierung eines KE (Unterabschnitt 6.4.1).

Ein gänzlich anderer Fall ist für die reflexive Partikelverbkonstruktion belegt: Ihr KE 〈WEG〉 ist, wie in Unterabschnitt 3.2.2 bereits dargestellt, als Nicht-Kern- 
KE zu charakterisieren. Es kann durch ein KtE instanziiert werden (was im Falle der Realisierung als PP eine Überschneidung mit der reflexiven Bewegungskonstruktion markiert, vgl. Unterabschnitt 3.2.3) oder uninstanziiert bleiben. Da ich die Leistung des Konstruktions-Frames für die reflexive Bewegungskonstruktion überwiegend an den KtE des KE WEG demonstriert habe, ist es also interessant zu untersuchen, wie sich der Anteil des Konstruktions-Frames an Konstrukt-Frames äußert, die keine FE zur Motivierung dieses KtE vorsehen. In Unterabschnitt 6.4.2 ziehe ich deshalb ergänzend zur bisherigen Analyse die Daten der reflexiven Partikelverbkonstruktion heran, um diesem Aspekt nachzugehen.

Zuletzt zeigen sich in den Daten Fälle, in denen der lexikalische Frame, der in einen Konstrukt-Frame eingeht, zwar als unrelatiert zu klassifizieren ist (da er nicht in Frame-Nähe zum Konstruktions-Frame Motion steht), ein Anteil des Konstruktions-Frames an entsprechenden Konstrukt-Frames aber, entgegen der bisherigen Analysen, nicht nachzuweisen ist. Dies ist für die reflexive Partikelverbkonstruktion sowie für die reflexive Bewegungskonstruktion belegt. In Unterabschnitt 6.4.3 möchte ich dieses ebenso zu Sonderfällen von Strukturparallelen zwischen Konstruktionen und Frames zählende Phänomen in den Blick nehmen.

\subsubsection{Mehrfache Instanziierung eines KE}

In Unterabschnitt 3.1.2 habe ich die reflexive Bewegungskonstruktion als aus vier Strukturelementen bestehend eingeführt: den drei KE BEWEGENDES, EREIGNIS und WeG sowie dem KEE. Die KtE des KE Weg können dabei, wie ich in Unterabschnitt 6.2.2 gezeigt habe, im Falle einer einfachen Motivierung durch den Konstruktions-Frame durch die FE Motion.GoAL, Motion.PATH, Motion.Source und Motion.DIRECTION einfach motiviert werden oder, wie in Unterabschnitt 6.2.3 gezeigt, unter Beteiligung ebendieser FE auch doppelt. Dass zwischen diesen FE eine Gemeinsamkeit besteht, ist nicht nur dadurch evident, dass sie das Inventar der möglichen FE des Konstruktions-Frames bilden, die für die Motivierung der KtE dieses KE infrage kommen, sondern auch dadurch, dass sie bereits innerhalb von Motion zusammenhängen und ein Core Set (Ruppenhofer et al. 2016: 25) bilden (dazu Unterabschnitt 7.3.3). Nun kommt es bisweilen vor, dass nicht nur eines dieser FE in einem Konstrukt instanziiert wird, sondern gleich mehrere.

Für Motion als lexikalischen Frame möchte ich dies an einigen Konstrukten zunächst illustrieren. Dabei sind verschiedene Kombinationen von FE möglich. Die Belege in (82) sind Beispiele für die Kombination aus Motion.SourcE und Motion.GoAL. 
(82) a. BÉDIÉ - er [Motion begab] sich am 3. Januar [Source von seinem Zufluchtsort in Togo aus] [GoAL nach Paris, wo er eine Wohnung besitzt] - wollte damit OUATTARA, der einen burkinabischen Vater hat, von einer Kandidatur ausschließen. (Archiv der Gegenwart, 2001 [2000])

b. HASSAN [Motion begab] sich Mitte Oktober in Begleitung einer tausend Mann starken bewaffneten Eskorte [Source von Djibouti aus] [GoAl nach Mogadischu], um seine Macht von dort aus auszuüben. (Archiv der Gegenwart, 2001 [2000])

c. Der Kommissionsentwurf [Motion bewegte] sich [Source vom anfänglichen Bestimmungslandprinzip] inzwischen [GoAL zu einem Herkunftslandprinzip], freilich mit vielen Ausnahmen, darunter „Maßnahmen“ (was immer das meint) zum Schutz der öffentlichen Ordnung, Gesundheit, Sicherheit - und der Verbraucher. (Die Zeit, 23.03.2000, Nr. 13)

Die Belege in (83) sind Beispiele für die Kombination aus den FE Motion.PATH und Motion.GoAL.

(83) a. Von einem Lkw aus gestartet, [Motion begibt] sich der zwei Meter lange Deltaflügler [РАTH auf programmierter Flugbahn] [GoAL in die Zielregion]. (Die Zeit, 17.02.2000, Nr. 8)

b. Das „wahrscheinlich“ gründete sich dabei ausschließlich auf das Vertrauen in die Überzeugungskraft seiner eigenen Argumente, die er am gleichen Tag Bismarck in einem Schreiben und am folgenden Tag dem Kaiser in einem ebensolchen vortrug, in dem er diesen zugleich um eine Audienz für einen Bevollmächtigten bat - er selber [Motion begab] sich in diesen Tagen ,auf ärztlichen Rat“ [ ${ }_{\mathrm{P}_{A T H}}$ über Italien] [GoAL nach Nizza]. (Gall, Lothar: Krupp, Berlin: Siedler 2000, S. 174)

Belegt sind indes nicht nur Kombinationen aus zwei FE, wie die soeben betrachteten, sondern ebenso Kombinationen aus drei FE. Die Belege in (84) sind Beispiele für die Instanziierung von Motion.SourCE, Motion.Direction und Motion.GoAL.

(84) a. Jonas [Motion wand] sich [Source aus dem Kofferraum] [Direction nach vorne] [GoAl auf die Rückbank des Autos]. (Glavinic, Thomas: Die Arbeit der Nacht, München Wien: Carl Hanser Verlag 2006, S. 346)

b. Schiller war kein Autor, der von innen kommt, er [Motion bewegte] sich [Direction in der Gegenrichtung], [Source von außen] [GoAL nach innen]. (Safranski, Rüdiger: Friedrich Schiller, München Wien: Carl Hanser 2004, S. 118) 
Eine zweite Kombination aus drei FE zeigen die beiden Belege in (85) mit den FE Motion.PATH, Motion.SourcE und Motion.GoAL.

a. Er [Motion schlängelt] sich [Source vom Hermon-Gebirge] [GoAL zum See Genezareth und zum Toten Meer], [РАтн durch ein Gebiet, das unter ständiger Trockenheit leidet]. (o. A. [kago]: Jordan. In: Aktuelles Lexikon 1974-2000, München: DIZ 2000 [2000])

b. Ein Drahtseil ist über den Fluß gespannt, mit einer beweglichen Winde ist das Fährboot daran festgetäut, und während die gewaltige Strömung auf das querstehende Ruder drückt, [Motion bewegen] sich Boot und Winde seitlich [РATH am Seil entlang] [SOURCE von einem Ufer] [GoAL zum andern]. (Düffel, John von: Vom Wasser, München: dtv 2006, S. 192)

Das Phänomen ist indes nicht allein auf Motion als lexikalischen Frame beschränkt, auch andere relatierte lexikalische Frames erlauben es. Die Belege in (86) sind Beispiele für den relatierten lexikalischen Frame Self_motion und die Kombination aus den FE Self_motion.SourcE und Self_motion.GoAL.

a. Ich [Self_motion hangelte] mich [Source von einem Zweijahresvertrag] [GoAL zum nächsten] und schrieb ab und zu Aufsätze. (Goosen, Frank: Liegen lernen, Frankfurt am Main: Eichborn AG 2000, S. 236)

b. Menschenaffen [self_motion hangeln] sich noch heute [Source von Ast] [GoAl zu Ast], unter elegantem Einsatz von Hand und Fuß. (Die Zeit, 23.03.2000, Nr. 13)

Auch für den relatierten lexikalischen Frame Body_movement ist eine ähnliche Kombination belegt, allerdings mit den beiden Nicht-Kern-FE Body_movement. $\langle$ SourCE $\rangle$ und Body_movement.(GoAL〉, wie die Beispiele in (87) zeigen.

a. Der Schläfer [Body_movement wälzte] sich wie üblich [〈SouRCE $\rangle$ von einer Seite] [〈GoAL $\rangle$ zur anderen]. (Glavinic, Thomas: Die Arbeit der Nacht, München Wien: Carl Hanser Verlag 2006, S. 172)

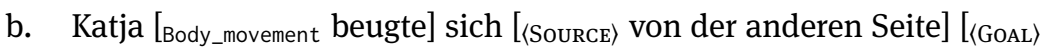
über ihren Bruder] und legte die Hand auf seine Stirn, als sei sie die Mutter. (Franck, Julia: Lagerfeuer, Köln: DuMont Literatur und Kunst Verlag 2003, S. 163)

Die mehrfache Instanziierung ein und desselben Elements in voneinander getrennten syntaktischen Konstituenten ist bereits in der traditionellen Grammatik bei Korrelaten wie dem Pronomen es bekannt (vgl. z.B. Duden 2016: 835-836, 1069-1071), konstruktionsgrammatisch wird ein solcher Fall als nominale Extra- 
position (Michaelis \& Lambrecht 1996) oder „[d]ouble instantiation“ (Fried 2015: 994) analysiert. Anders als bei Korrelaten ist es bei der mehrfachen Instanziierung eines KE allerdings nicht so, dass ,the properties of a single valence element are distributed over two discrete syntactic units" (Fried 2015: 994), sondern es sind gerade mehrere unterschiedliche FE, die instanziiert werden. Ihre Zusammengehörigkeit begründet sich darin, dass sie KtE motivieren, die zu einem KE zusammengefasst werden können. Die Notwendigkeit einer Unterscheidung zwischen KE und KtE einerseits und (deren Motivierung durch) FE andererseits zeigt sich hier besonders deutlich.

Argumentiert man zunächst rein frame-semantisch und nicht aus der Perspektive einer syntaktischen Konstruktion, so erscheinen diese Belege unproblematisch: Mehrere FE eines Frames können problemlos gemeinsam instanziiert werden. Lenkt man den Blick nun aber auf Konstrukte der reflexiven Bewegungskonstruktion, deren Konstrukt-Frames - anders als diejenigen der Belege in (82)(85) - nicht auf einem relatierten, sondern auf einem unrelatierten lexikalischen Frame basieren, braucht es einen anderen Erklärungsansatz. Für die bisher diskutierten Konstrukte der reflexiven Bewegungskonstruktion (Unterabschnitt 6.2.2 und 6.2.3) bin ich implizit davon ausgegangen, dass das KE WEG lediglich durch eine einzelne PP als KtE realisiert wird (vgl. Abbildung 6.2 in Unterabschnitt 6.1.2). Nun existieren allerdings analog zu den in (82)-(85) zitierten Belegen mit einem relatierten lexikalischen Frame solche, in deren Konstrukt-Frames ein unrelatierter Frame eingeht. Unter den - wenigen - Konstrukten, die dafür belegt sind, ist Beleg (88) ein Beispiel für die Kombination der FE Motion.SouRCE und Motion.GoAL mit dem lexikalischen Frame Rescuing und der LE retten (rescue.v).

(88) Mommsen [Rescuing rettete] sich [aus der Welt der Tatsachen Source] [in die der Gefühle ${ }^{\text {GoAL }}$ : (de Bruyn, Günter: Preußens Luise, Siedler 2001, S. 87)

Auch die Kombination aus Motion.Path und Motion.GoAL ist belegt, so für Beleg (89) für den lexikalischen Frame Smuggling und die LE schmuggeln (smuggle.v). In beiden Fällen liegt eine doppelte Motivierung mit Smuggling.PATH bzw. Smuggling.GoAL vor.

(89) Und wenn die Jungs nach getaner Tat in den Armen der Liebsten schlummern, [smuggling schmuggelt] sich Rudi mittels Dollars und Autogrammfußbällen [РАтн am Endlosstau von Brest vorbei ${ }^{\text {PATH }}$ [ [GoAL nach Polen ${ }^{\text {GOAL }}$ ] rein. (Die Zeit, 24.02.2000, Nr. 9)

Darüber hinaus findet sich auch ein Beleg (90), in dessen Konstrukt-Frame eines der FE des Konstruktions-Frames, Motion.GoAL, in doppelter Motivierung mit ei- 
nem FE des lexikalischen Frames Seeking für die LE tasten (grope.v) erscheint. Hinzu kommt separat das FE Motion.PATH in einfacher Motivierung.

(90) Die Frau [seeking tastet] sich [an der Wand entlang PATH] [Sought_entity zu der Alten ${ }^{\text {GoAL] }}$ hinüber und hockt sich schwerfällig neben sie hin. (Venske, Regula: Marthes Vision, Frankfurt am Main: Eichborn Verlag 2006, S. 189)

Wie ist mit diesen Befunden umzugehen? Die bisherige Annahme, das KE WEG wird durch eine einzige PP realisiert, kann vor diesem Hintergrund nicht mehr aufrechterhalten werden. Gleichzeitig stellen solche Phänomene aber auch die in diesem Kapitel entwickelte Vorstellung der semantischen Motivierung von KtE vor eine Herausforderung: Die Instanz eines KE wird hier nicht nur entweder durch ein FE des Konstruktions-Frames (einfach) oder durch jeweils ein FE des lexikalischen Frames und des Konstruktions-Frames (doppelt) motiviert, sondern es treten gleich mehrere FE des Konstruktions-Frames auf, teils sogar in doppelter Motivierung mit FE eines lexikalischen Frames. Wie gestalten sich die Strukturparallelen zwischen KtE und FE in diesen Fällen? Ich möchte dafür argumentieren, dass hier ein besonderer Fall der Instanziierung eines KE vorliegt, nämlich der, dass ein KE durch mehrere KtE instanziiert wird, die jeweils einfach oder doppelt motiviert werden. In den Belegen (88)-(90) - aber auch in allen für einen relatierten lexikalischen Frame - entspricht also jede Instanz eines FE wie Motion.GoAL, Motion.PATH, Motion.Source oder Motion.DiRECTION (ggf. in doppelter Motivierung mit einem FE eines unrelatierten lexikalischen Frames) einem KtE des KE WEG, das KE wird mithin mehrfach instanziiert. Dass es sich dabei zwar um mehrere KtE, aber um dasselbe KE, nämlich WEG, handelt, liegt offensichtlich daran, dass die vier FE von Motion zu einem Core Set gehören. Diese Eigenschaft der FE ist, wie ich in Unterabschnitt 7.3.3 näher diskutieren werde, eine wesentliche Voraussetzung, die Instanzen dieser vier FE als mehrere KtE eines KE zu betrachten.

Die Verhältnisse der mehrfachen Instanziierung eines KE lassen sich wie in Abbildung 6.6 schematisch darstellen. Die Pfeile von den FE des Frames Motion zum KE WEG der reflexiven Bewegungskonstruktion sollen andeuten, dass die semantische Definition des KE gewissermaßen eine Kumulation der Definitionen der FE darstellt, die entsprechend konstruktikographischen Niederschlag finden muss (dazu Unterabschnitt 7.3.3). Gleichzeitig wird durch die Pfeile vom KE zu den einzelnen KtE deutlich, dass für diesen Fall nicht ein FE die Motivierung eines einzigen KtE leistet, sondern dass mehrere FE zugleich in mehreren KtE instanziiert werden können.

Bei der in Unterabschnitt 3.1.2 eingeführten Struktur der reflexiven Bewegungskonstruktion mit vier Strukturelementen (BEWEGENDES, EREIGNIS, KEE 


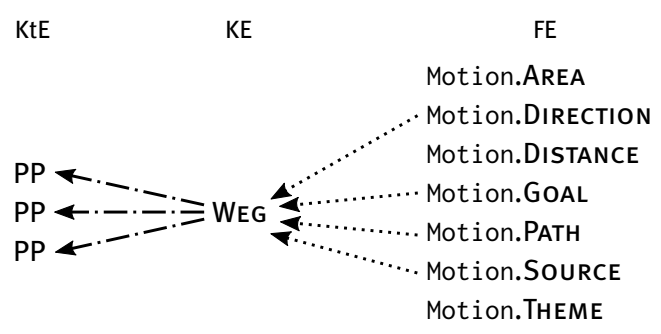

Abb. 6.6: Mehrfache Instanziierung des KE WEG der reflexiven Bewegungskonstruktion

und WEG) kann es somit bleiben. Die Anzahl der KE ändert sich in Konstrukten wie denen in (82)-(85) und (88)-(90) nicht, sondern lediglich die Anzahl der KtE, die für die reflexive Bewegungskonstruktion größer sein kann als die Anzahl der KE. Diese Besonderheit lässt sich durch den Konstruktions-Frame Motion erklären. Seine Struktur mit den vier FE, die zu einem Core Set gehören, macht diese Strukturerweiterung eines Konstrukts erst möglich. Selbstverständlich sie auch mit (relatierten) lexikalischen Frames möglich, die über ein analoges Core Set verfügen. $\mathrm{Zu}$ ihnen zählen etwa Self_motion (in Frame-Nähe von +1 in Vererbungsrelation) oder Fleeing (in Frame-Nähe von +2 in Vererbungsrelation).

Die Generalisierung des KE WEG im Rückgriff auf ein Core Set unterscheidet sich von derjenigen des FrameNet-Konstruktikons, das für die way-Konstruktion mit den FE Motion.Direction, Motion.Goal, Motion.Path und Motion.Source korrespondierende, einzelne KE ansetzt (vgl. Tabelle 2.4 in Unterabschnitt 2.3.2). ${ }^{81}$ Dieses Vorgehen halte ich aus mehreren Gründen für problematisch. Zunächst resultiert es im Konstruktionseintrag in einer inflationären Vermehrung von KE, die regelmäßig nicht instanziiert werden, denn die gleichzeitige Instanziierung der zu einem Core Set gehörenden FE ist für die reflexive Bewegungskonstruktion, insbesondere wenn es sich um unrelatierte lexikalische Frames handelt, ein eher seltenes Phänomen. Zudem missachtet die Ansetzung unterschiedlicher KE die Unterscheidung in relatierte und unrelatierte lexikalische Frames. Geht in den Konstrukt-Frame ein relatierter lexikalischer Frame ein, dessen FE sich von denen

\footnotetext{
81 Ähnlich gehen auch Ziem \& Flick (2019: 205, Anm. 5) und Ziem, Flick \& Sandkühler (2019: 68, Anm. 3) sowie Ziem (2020a: 27, Anm. 19) vor, wenn sie das zweite in einem Konstrukt instanziierte FE als Nicht-Kern-KE deuten. Diese Analyse schreibt jedoch einem der FE eines Core Sets den Status als Kern-KE zu, während es anderen den Status als Nicht-Kern-KE zuschreibt. Die Festlegung, welches FE den Kern-Status erhält, ist dann jedoch nicht nur potenziell arbiträr, sondern verschleiert auch den Fakt, dass jedes der FE in einem Core Set auch nur einmal instanziiert werden kann (vgl. die Analysen in Unterabschnitt 6.2.2). Somit müssen die FE des Core Sets gleichberechtigt behandelt werden, ein weiteres Argument für die Analyse einer mehrfachen Instanziierung eines KE WEG.
} 
von Motion hinreichend unterscheiden, sind manche dieser multiplen KE gänzlich obsolet. So verfügt etwa der Frame Body_movement, für den das Phänomen, wie in (87) zu sehen, ebenfalls belegt ist, nicht über ein FE ,DIRECTION`, das bei ihm als relatiertem lexikalischen Frame nicht instanziiert werden kann. Der Konstruktionseintrag würde dann ein KE vorsehen, das analog in diesem Fall nicht instanziierbar ist, was ihn unnötig überkomplex werden lässt und die Eigenschaften bestimmter lexikalischer Frames schlicht übergeht. Im ungünstigsten Fall würde dies zusätzlichen konstruktikographischen Aufwand bedeuten, da entsprechende Ausnahmen für bestimmte lexikalische Frames formuliert werden müssten.

Eine Generalisierung, die von der mehrfachen Instanziierung eines KE ausgeht, ist demgegenüber im Vorteil, weil sie ebendiese inflationäre Vermehrung von KE im Konstruktionseintrag vermeidet und gleichzeitig eine Generalisierung über unterschiedliche FE, die zu einem Core Set gehören, in einem KE ermöglicht - ein wesentliches Instrument zur Definition von KE (vgl. Unterabschnitt 7.3.3). Zudem wird dadurch überhaupt die Unterscheidung zwischen KE und (die Motivierung ihrer KtE durch) FE aufrecht erhalten, denn würden FE direkt als Äquivalenzen zu KE in den Konstruktionseintrag übernommen, würden die sich unterschiedlich ausprägenden Motivierungsverhältnisse, die ich in diesem Kapitel untersucht habe, gänzlich verwischt. Zuletzt kann die vorliegende Generalisierung für eine größere Zahl relatierter lexikalischer Frames Geltung beanspruchen, eben für solche, deren FE sich nicht direkt mit denjenigen von Motion parallelisieren lassen oder, wie Body_movement, gar nicht erst über bestimmte FE verfügen. Dass ein separater Konstruktions-Frame wie Motion für den Fall, dass die Motivierung aller KtE durch die FE eines relatierten lexikalischen Frames geleistet wird (Unterabschnitt 6.2.1) an einem Konstrukt-Frame gar keinen Anteil hat, wird damit offengelegt. Diese Zusammenhänge würden bei der Annahme multipler KE wie im FrameNet-Konstruktikon ignoriert.

\subsubsection{Nicht-Kern-KE und null-instanziierte FE}

Der zur mehrfachen Instanziierung eines KE, die ich soeben diskutiert habe, genau entgegengesetzte Fall betrifft KE, die in einigen Konstrukten der betreffenden Konstruktion einfach instanziiert werden, in anderen wiederum gar nicht. Die reflexive Beweungskonstruktion, die ich bisher als Beispiel für die Strukturparallelen zwischen Konstruktionen und Frames herangezogen habe, betrifft dies nicht, wohl aber die reflexive Partikelverbkonstruktion.

Wie bereits in Unterabschnitt 3.2.2 bemerkt, weist die reflexive Partikelverbkonstruktion ähnlich wie die reflexive Bewegungskonstruktion das KE 〈WEG〉 auf, mit dem Unterschied, dass es sich dabei um ein Nicht-Kern-KE handelt. An 
den Belegpaaren in (91)-(93) lässt sich erkennen, dass das KE 〈WEG〉 für die reflexive Partikelverbkonstruktion nicht obligatorisch ist, sondern fakultativ instanziiert werden kann. ${ }^{82}$ Es handelt sich um ein Nicht-Kern-KE im Sinne von Ziem \& Flick (2019: 205) und Ziem, Flick \& Sandkühler (2019: 67-68). Die jeweiligen a-Belege in (91)-(93) zeigen nun Konstrukte, in denen das KE uninstanziiert ist, während die b-Belege solche, in denen es instanziiert ist, zeigen. Besonders deutlich lässt sich diese Alternation zwischen uninstanziiertem und instanziiertem KE in Belegen mit derselben LE, die denselben lexikalischen Frame evoziert, beobachten. In (91) ist dies Self_motion mit der Basisverb-LE schleichen (sneak.v), in (92) Motion_directional mit der LE senken (descend.v) und in (93) Cause_to_move_in_place mit der LE drehen (turn.v).

(91) a. Im vierten Affärenmonat \{schleicht sich das Gift der Ermüdung ein\}. (Die Zeit, 24.02.2000, Nr. 9)

b. \{Sie schleichen sich ${ }_{\langle\mathrm{WEG}}{ }_{\mathrm{W}}$ ins Innere der Festung] ein\}. (Schwanitz, Dietrich: Männer, Frankfurt a. M.: Eichborn 2001, S. 295)

(92) a. Aus den sieben Uhr, wo wir die Insel hätten erreichen müssen, wurden acht, dann neun, halb zehn in Wolken, \{die sich immer weiter herabsenkten\}. (Schrott, Raoul: Tristan da Cunha oder die Hälfte der Erde; Hanser Verlag 2003, S. 440)

b. Er lügt zu jeder Zeit, dieser Nevskij-Prospekt, doch am meisten dann,

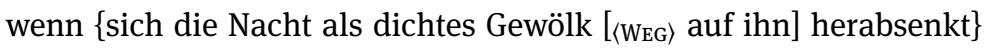
und die weißen und gelben Mauern der Häuser trennt, wenn sich die ganze Stadt in Donner und Blitz verwandelt, Myriaden Karossen sich über die Brücken wälzen, die Vorreiter schreien und auf ihren Pferden vorbeisprengen und wenn der Dämon selber die Lampen anzündet - einzig deshalb, um alles nicht in seiner wahren Gestalt zu zeigen. (Schlögel, Karl: Petersburg, München Wien: Carl Hanser Verlag 2002, S. 206)

(93) a. Barbara sagte „Okay“, \{drehte sich um\} und ging. (Goosen, Frank: Liegen lernen, Frankfurt am Main: Eichborn AG 2000, S. 132)

82 Dass es sich dabei tatsächlich um ein Charakteristikum dieser Konstruktion handelt, die auf den Gegensatz zwischen Partikelverben und simplizischen Verben zurückzuführen ist, bemerkt bereits Knobloch (2009: 552-553). Auch dies ist ein Grund, den formalen Aspekt der Instanziierung des KE EREIGNIS durch ein Partikelverb etwa im Namen der Konstruktion zu betonen (dazu auch Unterabschnitt 7.2.1). 
b. Dann fuhr er rechts ran, \{drehte sich [ ${ }_{\langle\mathrm{WEG}\rangle} \mathrm{zu}$ ihr] $\left.\mathrm{um}\right\}$ und fragte sie, ob sie nicht irgendwo was trinken gehen sollten. (Goosen, Frank: Liegen lernen, Frankfurt am Main: Eichborn AG 2000, S. 121)

Für eine Partikelverbkonstruktion wie die reflexive Partikelverbkonstruktion sind Fälle, in denen zusätzlich zur Verbpartikel eine direktionale PP, hier also ein KtE des KE 〈WEG〉 instanziiert wird, ungewöhnlich. Aus diesem Grund hat Olsen (1996b) für dieses Phänomen den Begriff des pleonastischen Direktionals eingeführt. ${ }^{83}$ Sie bezeichnet solche PP als pleonastisch, ,um den (von der Grammatik aus gesehen) redundanten Charakter, den sie im Zusammenhang mit einem Partikelverb zweifellos haben, hervorzuheben.“ (Olsen 1996b: 304). ${ }^{84}$ Für lexikalistisch ausgerichtete Grammatiktheorien sind pleonastische Direktionale, die zusammen mit Partikelverben auftreten, problematisch, weil die Verbpartikel bereits als ein Argument des Basisverbs gelten muss, sodass für die semantisch verwandte direktionale PP keine Argumentstelle mehr übrig bleibt (vgl. Olsen 1996b: 305, 1997b: 17, 1999: 232). Die damit verbundene Frage nach dem Lizenzierungsmechanismus pleonastischer Direktionale beantwortet Olsen (1996b: 304305, 1997b: 15, 1999: 232) dadurch, dass die PP als Modifikator der Verbpartikel auftritt und deren eher implizite semantische Eigenschaften expliziert.

Diese Beobachtungen lassen sich aus konstruktionssemantischer Perspektive einerseits einfach reformulieren, bedürfen andererseits aber einer Konkretisierung. Das erwähnte Lizenzierungsproblem ist unter der Annahme einer Konstruktion als eigenständiges ,Form-Bedeutungs-Paar' keines mehr: Die Lizenzierung eines pleonastischen Direktionals, im Falle der reflexiven Partikelverbkonstruktion als eines KtE des KE 〈WEG〉, findet nicht lexikalisch über das Verb, das als KtE des KE EREIGNIS in Erscheinung tritt, statt, sondern geschieht über die Konstruktion an sich. Selbst wenn die Argumentstruktur des Verbs keine Argumentstelle für ein Direktional vorsieht, kann diese weiterhin durch die Konstruktion beigetragen werden. Mehr noch: Nicht nur das Auftreten des pleonastischen KtE von $\langle$ WEG $\rangle$ kann durch die Konstruktion lizenziert werden, sondern ebenso das Auftreten der Verbpartikel (als Instanz des KEE RICHTUNG), mithin also der Wortbil-

83 Allerdings spricht bereits Harnisch (1982: 119) bei diesen Fällen von „pleonastisch oder zumindest redundant erscheinenden ,Doppelungen““. Das Phänomen selbst wird indes bereits von Kühnhold (1973: 184) beschrieben, die von einem „Valenzabbau gegenüber dem Grundverb“ (im Falle einer fehlenden Instanziierung des KE 〈WEG $\rangle$ ) spricht und gleichermaßen auf die Fakultativität der PP hinweist (vgl. Kühnhold 1973: 184).

84 In einem ähnlichen Kontext ist auch die Beobachtung von Kunze (1995: 22) für die reflexive Bewegungskonstruktion zu sehen, dass die direktionale PP (also das KE WEG) durch eine Verbpartikel ersetzt werden kann, was allerdings - ohne dass Kunze dies erwägt - den Übergang zur reflexiven Partikelverbkonstruktion markiert (vgl. dazu bereits Unterabschnitte 3.2.1 und 3.2.3). 
dungsprozess von einem simplizischen Basisverb hin zu einem Partikelverb. Beides lässt sich an Konstrukten der reflexiven Partikelverbkonstruktion, die einen unrelatierten lexikalischen Frame evozieren, illustrieren.

Bevor ich also auf die Strukturparallelen zwischen den KtE des KE 〈WEG〉 und entsprechenden FE eingehe und im Zuge dessen auf die Frage nach der Lizenzierung von Ersteren zurückkomme, sei ein Blick auf die Lizenzierung der Partikel (also des KEE RICHTUNG) gerichtet. In Konstrukten der reflexiven Partikelverbkonstruktion, die einen unrelatierten lexikalischen Frame evozieren, ist bereits für die Partikel keine Argumentstelle, d.h. kein FE im jeweiligen lexikalischen Frame, vorgesehen. Dieses muss also durch den Konstruktions-Frame bereitgestellt werden. Als FE kommt hierfür Motion.DiRECTION infrage, auf die tendenziell unspezifische Richtung der im Konstrukt ausgedrückten ,Bewegung' hinweist und die wesentliche Motivation für die Benennung des KEE mit Richtung ist (vgl. dazu schon Unterabschnitt 3.2.2). Fälle, in denen die Partikel nicht durch den lexikalischen Frame allein syntaktisch lizenziert und damit semantisch motiviert werden kann, sind eine wesentliche Motivation dafür, sie als eigenständiges KEE anzunehmen.

Beispiele, in denen sich Motivierungen der Partikel durch den KonstruktionsFrame finden lassen, sind größtenteils analog zur einfachen Motivierung der KtE des KE WEG der reflexiven Bewegungskonstruktion (Unterabschnitt 6.2.2) und betreffen lexikalische Frames wie Daring (94), Hostile_encounter (95), Chatting (96), Work (97) oder Ingestion (98).

(94) a. Sie [Daring wagte] sich allerdings nur [hinein ${ }^{\text {DIRECTION] }}$, wenn jemand dabei war, entweder Minna, ihre große Schwester, oder ihre Mutter. (Pressler, Mirjam: Malka Mai, Weinheim Basel: Beltz \& Gelberg 2001, S. 6)

b. Obwohl nach den Untersuchungen der Uno und der OECD fast alle EU-Staaten Einwanderer brauchen werden, [Daring wagt] sich im Augenblick niemand [vor Direction]. (Die Zeit, 02.03.2000, Nr. 10)

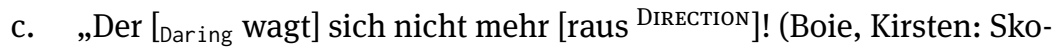
gland, Ort: Hamburg 2005, S. 202)

(95) a. Mühsam [Hostile_encounter kämpft] sich dieses Ein-Milliarden-Volk [voran Direction]. (Weizsäcker, Richard von: Dreimal Stunde Null? 1949 1969 1989, Berlin: Siedler Verlag 2001, S. 186)

b. Ich [Hostile_encounter kämpfte] mich [hinaus ${ }^{\text {DiRECTion] }}$ und stieg im Garten den Hang hinauf. (Schulze, Ingo: Neue Leben, Berlin: Berlin Verlag 2005, S. 159)

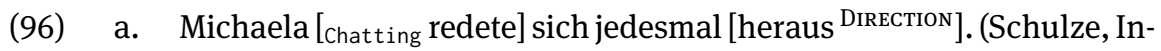
go: Neue Leben, Berlin: Berlin Verlag 2005, S. 577) 


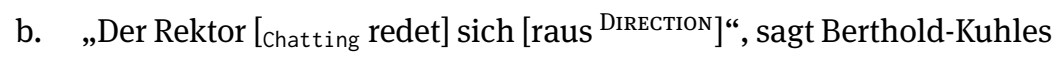
und sieht sich mittlerweile vor der Frage, „ob es für mich nicht günstiger ist, zum Sozialamt zu gehen“. (Die Zeit, 13.04.2000, Nr. 16)

(97) Breiring kam ins Krankenhaus, Eichhorn hatte Semesterferien, er über-

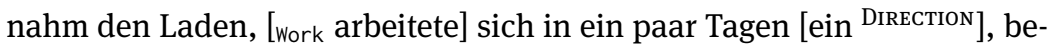
nutzte die Karte und die Fähnchen bald so geläufig wie jedes andere einleuchtend konstruierte Werkzeug. (Kopetzky, Steffen: Grand Tour, Frankfurt am Main: Eichborn 2002, S. 154)

(98) Der Gestank, der trotz des Regens in der Luft liegt, ist so widerlich, daß sogar die Fliegen fortbleiben; nur die Ratten vermehren sich, [Ingestion fressen] sich [durch ${ }^{\text {DIRECTION] }}$ und bauen sich Nester in den fauligen Eingeweiden. (Schrott, Raoul: Tristan da Cunha oder die Hälfte der Erde; Hanser Verlag 2003, S. 256)

In solchen Konstrukten wird die Partikel als Instanz des KEE RICHTUNG nicht durch den lexikalischen Frame, sondern durch den Konstruktions-Frame motiviert, da im lexikalischen Frame kein FE für ihre Motivierung angelegt ist. Das FE Motion.DIRECTION ergänzt somit den ansonsten unvollständigen KonstruktFrame. Darüber hinaus kann die Frage gestellt werden, ob die Partikel dafür verantwortlich gemacht werden kann, den Konstruktions-Frame überhaupt erst zu evozieren (dazu Unterabschnitt 8.3.1).

Für die fakultative Instanziierung des Nicht-Kern-KE 〈WEG) gelten nun dieselben Prinzipien wie für die Lizenzierung der Partikel. Relatierte lexikalische Frames wie diejenigen, die in Konstrukten wie denen in (91) und (92) den KonstruktFrame konstituieren, stellen bereits ein FE bereit, das durch ein KtE von 〈WEG) instanziiert wird. Die Zusammenhänge sind hier also analog zu der Instanziierung des KE WEG der reflexiven Bewegungskonstruktion, die ich in Unterabschnitt 6.2.1 diskutiert habe.

Ebenso analog ist die Instanziierung des Nicht-Kern-KE $\langle\mathrm{WEG}\rangle$ der reflexiven Partikelverbkonstruktion, die sich in Konstrukten mit einem unrelatierten lexikalischen Frame beobachten lässt. Die folgenden Belege sind Beispiele für die bereits diskutierten lexikalischen Frames Daring (99), Hostile_encounter (100), Work (101) und Ingestion (102).

(99) a. Wie die Franzosen platzierten sie ihre Anlagen an den attraktivsten Stränden rund ums Mittelmeer und [Daring wagten] sich bald auch [auf andere sonnenverwöhnte Erdteile $\left.{ }^{\mathrm{GOAL}}\right]$ vor, in denen immer sommerliche Temperaturen herrschten. (Die Zeit, 09.03.2000, Nr. 11)

b. Aber früher, als wir noch klein waren, [Daring traute] sich niemand [an uns ${ }^{\text {GOAL }}$ ] heran, auch nicht in Mückes Gegend, denn wir standen un- 
ter dem Schutz seines Bruders, und dessen Rache würde fürchterlich sein. (Goosen, Frank: Liegen lernen, Frankfurt am Main: Eichborn AG 2000, S. 88)

c. Er meidet diese Gegend, [Daring wagt] sich nicht [auf den Hügel GoAL] herauf, fürchtet noch den entferntesten Blickkontakt mit einem Mitglied seiner Familie. (Beyer, Marcel: Spione, Köln: DuMont 2000, S. 126)

d. Und doch erschrickt man bei der neuen CD des Altmeisters, der sich von der Musik weit vor[Daring wagt] [in Meditation und Glaubenswelten ${ }^{\text {GoAL }}$ ], wie er es in seinem jüngsten Buch dokumentiert: (Die Zeit, 27.01.2000, Nr. 5)

(100) a. Die überrumpelte Frau will, man merkt es ihr an, keine Spielverderberin sein und [Hostile_encounter ringt] sich gegen spürbare Widerstände [zum Mitmachen GoAL] durch. (Die Zeit, 20.01.2000, Nr. 4)

b. Das von Uganda unterstützte MLC BEMBAs, mittlerweile eine der größten Rebellengruppen, kontrolliert weite Gebiete im Osten und Norden des Kongo und [Hostile_encounter kämpft] sich [in Richtung der Hauptstadt Kinshasa DiRection] vor. (Archiv der Gegenwart, 2001 [2000])

c. Nass geschwitzt stiegen sie wieder zur Straße hinauf - nachdem Staubfinger dem starrköpfigen Wagen einen letzten Tritt versetzt hatte -, kletterten über die Mauer, die aussah, als wäre jeder einzelne Stein mehr als tausend Jahre alt, und [Hostile_encounter kämpften] sich [den Hang ${ }^{\mathrm{PATH}}$ ] hinauf. (Funke, Cornelia: Tintenherz, Hamburg: Cecilie Dressler Verlag 2003, S. 226)

d. Sie haben die Menschenrechte zur Richtschnur der Außenpolitik erklärt - und [Hostile_encounter ringen] sich [zu keiner Verurteilung des Tschetschenien-Krieges GoAL] durch. (Die Zeit, 23.03.2000, Nr. 13)

(101) Sein Herz [work arbeitete] sich voran, immer höher, immer dichter [unter die Haut ${ }^{\text {GoAL }}$. (Schulze, Ingo: Neue Leben, Berlin: Berlin Verlag 2005, S. 751)

(102) Frisch beieinander sollten die Kleider der beiden verhindern, daß er sich [aus dieser Ehe Source] heraus[Ingestion säuft]. (Müller, Herta: Der König verneigt sich und tötet, München: Carl Hanser Verlag 2003, S. 8)

In Konstrukten wie diesen werden sowohl die Partikel als auch die KtE des KE 〈WEG〉 durch den Konstruktions-Frame Motion motiviert. Die Partikel wird stets durch das FE Motion.DiREcTION motiviert, während die Motivierung der KtE des $\mathrm{KE}\langle\mathrm{WEG}\rangle$ wie in der reflexiven Bewegungskonstruktion für das KE WEG variiert. 
Tab. 6.13: Konstrukte der reflexiven Partikelverbkonstruktion ohne Instanziierung des NichtKern-KE 〈WEG

\begin{tabular}{lrr}
\hline Lexikalischer Frame & Konstrukte & Anteil \\
\hline Relatiert & 168 & $36,13 \%$ \\
Unrelatiert & 297 & $63,87 \%$ \\
\hline Gesamt & 465 & $100,00 \%$ \\
\hline
\end{tabular}

Tab. 6.14: Konstrukte der reflexiven Partikelverbkonstruktion mit Instanziierung des Nicht-KernKE 〈WEG

\begin{tabular}{lrr}
\hline Lexikalischer Frame & Konstrukte & Anteil \\
\hline Relatiert & 136 & $35,32 \%$ \\
Unrelatiert & 249 & $64,68 \%$ \\
\hline Gesamt & 385 & $100,00 \%$ \\
\hline
\end{tabular}

Aber noch immer unbeantwortet bleibt die Frage, warum das KE in manchen Konstrukten instanziiert wird, in anderen aber nicht. Eine Antwort darauf muss den Zusammenhang zwischen der semantischen Motivierung der Partikel als Instanz des KEE RICHTUNG und derjenigen der KtE des KE 〈WEG〉 adressieren, was zugleich eine detailliertere Untersuchung der FE, die für diese Motivierung infrage kommen, erfordert.

Wie bereits aus der Diskussion um die pleonastische Natur der KtE des KE $\langle$ WEG $\rangle$ abzuleiten ist, liegt es nahe anzunehmen, dass die fehlende Instanziierung dieses Nicht-Kern-KE den prototypischen Fall darstellt, während die durch seine Instanziierung ausgelöste ,doppelte‘ Kodierung desselben semantischen Aspekts einen selteneren Fall darstellt. Anhand der Daten zur reflexiven Partikelverbkonstruktion lässt sich diese Annahme überprüfen. Wie die Tabellen 6.13 und 6.14 zeigen, bestätigt sie sich: Die Mehrheit der insgesamt 850 Konstrukte dieser Konstruktion weist eine fehlende Instanziierung des KE (WEG) auf (465 Konstrukte), während die Instanzierung des KE 〈WEG〉 quantitativ niedriger liegt (385 Konstrukte).

Wie lassen sich diese Beobachtungen, sowohl die Instanziierung des KE als auch dessen frequentere fehlende Instanziierung, semantisch deuten? Um eine Antwort zu bekommen, ist es vonnöten, noch einmal den Zusammenhang zwischen der Partikel und der Instanziierung des KE 〈WEG〉 in den Blick zu nehmen. Für Olsen (1996b) stellt sich der Zusammenhang zwischen Partikel und pleonastischem Direktional wie folgt dar: 
Die Partikeln [...] unterscheiden sich von den verwandten vollen Präpositionalphrasen [...] lediglich dadurch, daß die Partikeln das Ziel (bzw. im Fall von durch das Durchgangsobjekt) der Bewegung implizit lassen, während es in der Präpositionalphrase explizit zum Ausdruck kommt. (Olsen 1996b: 304)

Aus frame-semantischer Perspektive ist dieses Implizitlassen der durch die Instanziierung des KE $\langle\mathrm{WEG}\rangle$ kodierten Information leicht zu erfassen. Es handelt sich um eine Null-Instanziierung des entsprechenden FE, genauer gesagt: eine definite Null-Instanziierung (DNI). Definite Null-Instanziierung liegt laut Ruppenhofer et al. (2016: 28) in Fällen vor, ,in which the missing element must be something that is already understood in the linguistic or discourse context." Sie betreffen hinsichtlich des Konstruktions-Frames für das KE 〈WEG〉 der reflexiven Partikelverbkonstruktion ebenso wie für das KE WEG der reflexiven Bewegungskonstruktion die ein Core Set bildenden Kern-FE Motion.SourCE, Motion.Path, Motion.Goal und Motion.DiRECTION. Interessanterweise argumentiert schon Olsen (1999) für die Weglassung pleonastischer Direktionale in einer an den Begriff der definiten Null-Instanziierung erinnernden Weise, wenn sie feststellt, ${ }^{85}$

daß der Wert des implizit gelassenen Relatums der zugrundeliegenden Präpositionalrelation aus dem Kontext zu ergänzen ist - meist dadurch, daß entweder ein im Kontext prominent vorhandenes oder zumindest ein für den entsprechenden Situationstyp typisches Objekt aufgrund konzeptuellen Wissens logisch und pragmatisch dafür in Frage kommt. (Olsen 1999: 227$)^{86}$

Konstruktikographisch setzen Ziem \& Flick (2019: 205) sowie Ziem, Flick \& Sandkühler (2019: 67-68) für potenziell von Null-Instanziierungen betroffene KE die bereits angesprochene Unterscheidung von Kern-KE und Nicht-Kern-KE an, die unverkennbar an FrameNets Unterscheidung zwischen Kern-FE und Nicht-KernFE angelehnt ist. Werden Kern-KE weggelassen, handelt es sich um eine NullInstanziierung, Nicht-Kern-KE können wie Nicht-Kern-FE frei weggelassen werden (Unterabschnitt 2.1.2). Das KE 〈WEG〉 der reflexiven Partikelverbkonstruktion ist in dieser Unterscheidung also als Nicht-Kern-KE zu verstehen. Problematisch

85 Allerdings macht bereits Mungan (1986: 59) eine ähnliche Beobachtung der Weglassbarkeit eines Direktionals, wenn dieses im situationalen Kontext mitverstanden wird.

86 An anderer Stelle spricht Olsen (1997a: 124) davon, „daß die Partikelbedeutung, die ein implizites (d. h. existentiell abgebundenes) Argument beinhaltet, durch eine vollständige Präpositionalphrase mit meist kongruenter Präposition näher expliziert werden kann. "Der Verweis auf die existenzielle Deutung des Direktionals ließe prinzipiell auch eine Analyse als indefinite NullInstanziierung (INI) zu (vgl. dazu die Definition von Ruppenhofer et al. 2016: 28-29). Da aber die overt realisierte Partikel als ko(n)textueller Indikator für den im KtE von 〈WEG〉 ausgedrückten semantischen Aspekt dient, bleibe ich bei einer Analyse als DNI. 
ist an dieser Terminologie einzig die Tatsache, dass die FE, die für die semantische Motivierung der KtE des KE 〈WEG〉 im Falle der reflexiven Partikelverbkonstruktion gerade keine Nicht-Kern-FE sind, wie es die terminologische Parallele suggerieren könnte. Es handelt sich dabei im Falle des Konstruktions-Frames um die ein Core Set bildenden FE Motion.Source, Motion.PATH, Motion.GoAL und Motion.DIRECTION, die Kern-FE sind. Auf sie ist der Begriff der Null-Instanziierung also problemlos anzuwenden, für Nicht-Kern-KE ist er hingegen (wie bei NichtKern-FE) gerade nicht vorgesehen: ${ }^{87}$

Note that for some constructions it is necessary to distinguish between core and non-core CEE. [sic!] While core CEs have to be realized unless their omission is licensed by a null instantiation mechanism, instantiations of non-core CEs are optional [...]. (Ziem, Flick \& Sandkühler 2019: 68).

Auf der Ebene von KE kann also nicht von Null-Instanziierung gesprochen werden, wenn es sich dabei nicht um Kern-KE handelt. Indes können die FE, die potenziell der Motivierung eines KtE dienen, im Falle der fehlenden Instanziierung eines Nicht-Kern-KE sehr wohl null-instanziiert werden (wie für den KonstruktionsFrame Motion). Trotz dieser Diskrepanz zwischen der Instanziierung bestimmter KE und der semantischen Motivierung ihrer KtE ist die Weglassbarkeit eines NichtKern-KE Anlass genug, es als solches zu bezeichnen, solange deutlich gemacht wird, dass damit nicht eine Strukturparallele zu Nicht-Kern-FE impliziert ist.

Wird ein Nicht-Kern-KE wie 〈WEG〉 der reflexiven Partikelverbkonstruktion nicht instanziiert, handelt es sich, wie gesehen, um eine definite Null-Instanziierung des FE, das die potenziellen KtE des KE motivieren kann. Die durch das entsprechende KtE von $\langle\mathrm{WEG}\rangle$ realisierbare semantische Information ist aus dem Kontext zu verstehen, weil bereits die Partikel darauf hinweist, dass ein solcher semantischer Aspekt kodiert ist. Dies leitet noch einmal über zu der Frage, welche FE für die Motivierung der Partikel und möglicher KtE des KE 〈WEG〉 infrage kommen. Wie bereits erwähnt, gehe ich davon aus, dass die Partikel stets durch das FE Motion.DiRECTION motiviert wird. Sie zeigt an, dass die in der Konstruktbedeutung enthaltene ,Bewegung‘ in eine bestimmte ,Richtung‘ verläuft, lässt die Details dieser ,Richtung‘ aber offen. Zu diesen Details gehören Informationen wie der ,Ursprung‘ der ,Bewegung‘ (Motion.Source), der ,Weg‘, auf dem die ,Bewegung‘ verläuft (Motion.PATH) oder der ,Endpunkt‘ der ,Bewegung‘ (Motion.GoAL).

87 Wichtig ist hier, dass der Null-Instanziierung nicht einzelne FE des Core Set unterworfen sind, sondern das Core Set als Ganzes: Sobald eines der FE des Core Set instanziiert wird, müssen die anderen nicht instanziiert sein (vgl. Ruppenhofer et al. 2016: 25), weshalb sie dann nicht von einer Null-Instanziierung betroffen sind. Ich komme auf diesen Aspekt in Unterabschnitt 7.3.3 zurück. Zu Problemen bei der Annotation von null-instanziierten Core Sets vgl. Dux (2020: 72-73). 
Diese Details können nun durch eine Instanziierung des KE 〈WEG〉, dessen KtE durch eines der FE im Core Set overt ausgedrückt und damit spezifiziert werden. ${ }^{88}$

Frame-semantisch gesehen ist die höhere Frequenz an Konstrukten ohne die Instanziierung von $\langle\mathrm{WEG}\rangle$ und mit einer Null-Instanziierung eines entsprechenden FE (vgl. Tabelle 6.13) keine Überraschung: „Im Gegensatz zu einem weit verbreiteten linguistischen Vorurteil ist ,Implizitheit' [...] der Normalfall, und keineswegs die Ausnahme (wie es in fast allen gängigen semantischen und syntaktischen Theorien unterstellt wird.“ (Busse 2012: 645-646). ${ }^{89}$ Lönneker (2003: 67) schreibt dazu: „Auch die Abwesenheit bestimmter Eigenschaften und Relationen kann einen wichtigen Teil des Weltwissens darstellen“. Ganz ähnlich ist bei Fraas (2005: 246) zu lesen: „Auch das Frei-Bleiben von Slots [...] ist ein wichtiger Befund und provoziert die Frage nach den Gründen." FrameNet bietet mit dem Konzept der Null-Instanziierung einen möglichen Grund an: Liegt eine DNI vor, ist die sprachlich unausgedrückte Information im Kontext bereits mitzuverstehen, ${ }^{90}$ aber nicht relevant genug, um overt ausgedrückt zu werden. Um die Frage zu beantworten, was genau aus dem Kontext mitverstanden werden muss, ist es sinnvoll, sich diejenigen Konstrukte anzusehen, in denen die Information sprachlich ausgedrückt ist; für die reflexive Partikelverbkonstruktion also jene, in denen das $\mathrm{KE}\langle\mathrm{WEG}\rangle$ instanziiert ist. Die semantische Motivierung seiner KtE gibt also darüber Aufschluss, welche Informationen in den Konstrukten ohne instanziiertes KE am häufigsten unausgedrückt bleibt.

Ein Blick auf die Motivierung der KtE des instanziierten KE 〈WEG〉 ist deshalb ebenso für die Interpretation der Konstrukte ohne dessen Instanziierung interessant. Sieht man sich die FE des Konstruktions-Frames Motion an, die in die Mo-

88 Eine systematische Betrachtung der Beziehungen zwischen dem FE Motion.Direction, das die Partikel motiviert und den FE Motion.SourCE, Motion.PATH und Motion.GoAl würde eine separate Repräsentationsebene innerhalb der konstruktikographischen Beschreibung verlangen, die in etwa den von Barsalou (1992b: 35-37, 1993: 39) postulierten strukturellen Invarianten entsprächen, die in der Lage sind, Relationen zwischen den FE eines Frames zu erfassen. Relationen dieser Art bezeichnet Busse (2012: 629) auch als Intra-Frame-Relationen in Kontrast zu den in FrameNet überwiegend dokumentierten Inter-Frame-Relationen. Aus Gründen der Übersichtlichkeit verzichte ich auf eine Modellierung solcher Relationen, zumal bereits an der quantitativen Verteilung der FE, die das KE $\langle\mathrm{WEG}\rangle$ motivieren, gewisse Präferenzen zu erkennen sind, die in die konstruktionsspezifische Formulierung struktureller Invarianten für Motion eingehen müssten. 89 Busse stützt sich hier auf Bartlett (1932: 195). An anderer Stelle bringt Busse (2012: 42) diese Idee als Schlussfolgerung aus den Überlegungen zu einer Salienz-Hierarchie semantischer Rollen von Fillmore (1977b: 80) in das Postulat eines minimalistischen Kommunikationsmodells. Vgl. auch die Systematisierungsversuche von hintergründigen Bezugsobjekten bei von Polenz (2008: 130-137).

90 Zum Begriff des Mitverstehens vgl. von Polenz (2008: 302-307) 
tivierung der KtE des instanziierten KE 〈WEG involviert sind (Tabelle 6.15), ist eine deutliche Präferenz zugunsten des FE Motion.GoAL zu beobachten, die bereits in Unterabschnitt 6.2.2 analog für das KE WEG der reflexiven Bewegungskonstruktion festgestellt werden konnte. ${ }^{91}$ Damit ist unschwer zu erkennen, dass die durch die Partikel und deren Motivierung mit Motion.DiREcTION zunächst implizit ausgedrückte ,Richtung‘ der ,Bewegung‘ am häufigsten auf ihr ,Ziel` hin spezifiziert wird. Gleichzeitig lässt dies darauf schließen, dass in Konstrukten, in denen das $\mathrm{KE}$ 〈WEG $\rangle$ uninstanziiert bleibt, am häufigsten jene Information über das ,Ziel` der ,Bewegung‘ implizit bleibt und damit mitverstanden werden muss.

An einigen der als (99)-(102) zitierten Belege, die ich in (103)-(106) wiederhole, lassen sich diese Zusammenhänge verdeutlichen, indem man die Motivierungen der Partikel und derjenigen der KtE des $\mathrm{KE}\langle\mathrm{WEG}\rangle$ zugleich betrachtet.

(103) a. Wie die Franzosen platzierten sie ihre Anlagen an den attraktivsten Stränden rund ums Mittelmeer und [Daring wagten] sich bald auch [auf andere sonnenverwöhnte Erdteile GoAL] [vor Direction], in denen immer sommerliche Temperaturen herrschten. (Die Zeit, 09.03.2000, Nr. 11)

b. Aber früher, als wir noch klein waren, [Daring traute] sich niemand [an uns GoAL] [heran Direction], auch nicht in Mückes Gegend, denn wir standen unter dem Schutz seines Bruders, und dessen Rache würde fürchterlich sein. (Goosen, Frank: Liegen lernen, Frankfurt am Main: Eichborn AG 2000, S. 88)

c. Er meidet diese Gegend, [Daring wagt] sich nicht [auf den Hügel Goal] [herauf ${ }^{\text {DiREction] }}$, fürchtet noch den entferntesten Blickkontakt mit einem Mitglied seiner Familie. (Beyer, Marcel: Spione, Köln: DuMont 2000, S. 126)

d. Und doch erschrickt man bei der neuen CD des Altmeisters, der sich von der Musik weit [vor Direction][Daring wagt] [in Meditation und Glaubenswelten ${ }^{\mathrm{GOAL}}$ ], wie er es in seinem jüngsten Buch dokumentiert: (Die Zeit, 27.01.2000, Nr. 5)

91 Tabelle 6.15 enthält die Anzahl der Konstrukte, in denen die KtE des instanziierten KE 〈WEG) einfach durch den lexikalischen Frame Motion, den Konstruktions-Frame Motion (bei einem unrelatierten lexikalischen Frame) sowie doppelt motiviert wird. Die Summe der Konstrukte liegt damit höher als diejenige der Konstrukte mit unrelatierten Frames und Instanziierung des KE $\langle\mathrm{WEG}\rangle$, die in Tabelle 6.14 dargestellt ist. 
Tab. 6.15: FE des Konstruktions-Frames Motion in einfacher und doppelter Motivierung der KtE des instanziierten KE 〈WEG〉 der reflexiven Partikelverbkonstruktion

\begin{tabular}{lrrrr}
\hline & Motion.Goal & Motion.PatH & Motion.SOURCE & Motion.DiRection \\
\hline$a b$ & 2 & 2 & 0 & 0 \\
an & 11 & 0 & 0 & 0 \\
auf & 4 & 5 & 0 & 0 \\
aus & 1 & 0 & 2 & 0 \\
bei & 0 & 4 & 0 & 0 \\
durch & 11 & 6 & 0 & 0 \\
ein & 23 & 0 & 0 & 0 \\
fort & 1 & 1 & 0 & 0 \\
frei & 0 & 0 & 0 & 0 \\
heim & 1 & 0 & 0 & 0 \\
her & 0 & 0 & 0 & 0 \\
hin & 3 & 0 & 0 & 0 \\
hinter & 0 & 0 & 0 & 0 \\
hoch & 0 & 0 & 0 & 0 \\
mit & 0 & 0 & 0 & 0 \\
nach & 0 & 0 & 0 & 0 \\
über & 0 & 0 & 0 & 0 \\
um & 0 & 1 & 0 & 0 \\
unter & 0 & 1 & 1 & 0 \\
vor & 8 & 3 & 1 & 1 \\
weg & 0 & 0 & 0 & 0 \\
zu & 7 & 0 & 1 & 0 \\
zurück & 3 & 0 & 5 & 0 \\
\hline Gesamt & 75 & 23 & & 0 \\
\hline & & & 0 & 0
\end{tabular}

(104) a. Die überrumpelte Frau will, man merkt es ihr an, keine Spielverderberin sein und [Hostile_encounter ringt] sich gegen spürbare Widerstän-

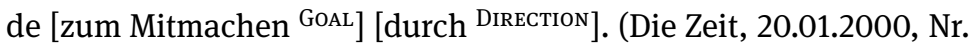
4)

b. Nass geschwitzt stiegen sie wieder zur Straße hinauf - nachdem Staubfinger dem starrköpfigen Wagen einen letzten Tritt versetzt hatte -, kletterten über die Mauer, die aussah, als wäre jeder einzelne Stein mehr als tausend Jahre alt, und [Hostile_encounter kämpften]

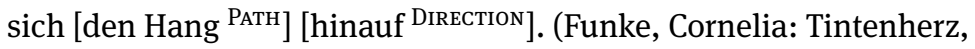
Hamburg: Cecilie Dressler Verlag 2003, S. 226)

c. Sie haben die Menschenrechte zur Richtschnur der Außenpolitik erklärt - und [Hostile_encounter ringen] sich [zu keiner Verurteilung des 
Tschetschenien-Krieges GoAL] [durch Direction]. (Die Zeit, 23.03.2000, Nr. 13)

(105) Sein Herz [work arbeitete] sich [voran DiREction], immer höher, immer dichter [unter die Haut ${ }^{\text {GoAL }}$ ]. (Schulze, Ingo: Neue Leben, Berlin: Berlin Verlag 2005, S. 751)

(106) Frisch beieinander sollten die Kleider der beiden verhindern, daß er sich [aus dieser Ehe Source] [heraus ${ }^{\text {DiREction] }}$ [Ingestion säuft]. (Müller, Herta: Der König verneigt sich und tötet, München: Carl Hanser Verlag 2003, S. 8)

Die Beobachtungen, dass die KtE des KE 〈WEG〉 die durch die Partikel ausgedrückte ,Richtung، (Motion.DiRECTION) näher spezifizieren, kann somit auf die Annahme zurückgeführt werden, „daß die Partikelbedeutung, die ein implizites Argument enthält, durch eine vollständige Präpositionalphrase näher expliziert werden kann.“ (Olsen 1999: 227). Diese Explikation wird immer dann geleistet, wenn die bereits durch das FE Motion.DIRECTION motivierte Partikel durch ein KtE des $\mathrm{KE}$ 〈WEG〉 ergänzt wird, das ein anderes FE zum Konstrukt-Frame hinzufügt. Da auf der Ebene eines Frames nicht zwischen der impliziten Instanziierung eines FE (durch die Partikel, also das KEE RICHTUNG) und der expliziten Instanziierung eines FE (durch eine PP, also durch ein KtE des KE 〈WEG〉) unterschieden werden kann, muss der Zusammenhang zwischen beiden also dadurch dargestellt werden, dass es sich um FE desselben Core Set handelt.

Eine Besonderheit ergibt sich allerdings, wenn in einem Konstrukt sowohl die Partikel als auch das KtE des KE 〈WEG〉 durch das FE Motion.DIRECTION motiviert sind. Entgegen des von Fillmore (1968: 21) postulierten Prinzips, dass jede semantische Rolle nur ein einziges Mal in einem Satz instanziiert werden kann, wird das betreffende FE somit zweimal instanziiert. Ein Beispiel liegt mit Beleg (107) vor.

Das von Uganda unterstützte MLC BEMBAs, mittlerweile eine der größten Rebellengruppen, kontrolliert weite Gebiete im Osten und Norden des Kongo und [Hostile_encounter kämpft] sich [in Richtung der Hauptstadt Kinshasa Direction] [vor Direction]. (Archiv der Gegenwart, 2001 [2000])

In Fällen wie diesen ist also die Annahme, dass dasselbe FE sowohl durch die Partikel als auch durch ein KtE des KE 〈WEG〉 instanziiert werden kann, unumgänglich. Strenggenommen handelt es sich im Falle der fehlenden Instanziierung von 〈WEG $\rangle$ in diesen Fällen dann nicht um eine vollständige Null-Instanziierung, da das FE ja implizit bereits durch die Partikel instanziiert wird, eine vollständige Spezifizierung seines Füllwertes allerdings ausbleibt. Die Instanziierung der Partikel ohne ein durch Motion.DIRECTION motiviertes KtE des KE 〈WEG〉 wäre so- 


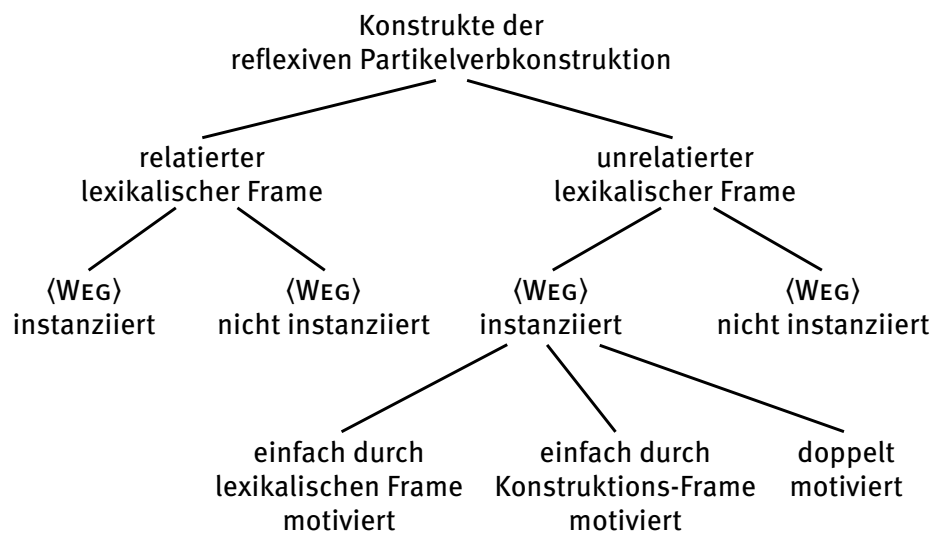

Abb. 6.7: Verteilung der Konstrukte der reflexiven Partikelverbkonstruktion nach Instanziierung des KE 〈WEG

mit auf einem Kontinuum zwischen der vollständigen Null-Instanziierung des betreffenden FE und seiner vollständigen Instanziierung durch einen entsprechend konkreten Füllwert etwa in der Mitte zu lokalisieren. Da die aktuelle Annotationspraxis von FrameNet ebenso wie der Konstruktikographie keine Möglichkeit bietet, dies darzustellen, muss im Falle der Instanziierung des KE 〈WEG〉 hier auf eine doppelte Annotation desselben FE zurückgegriffen werden.

Dadurch, dass es sich bei 〈WEG〉 um ein Nicht-Kern-KE handelt, gestaltet sich die Verteilung aller Konstrukte dieser Konstruktion hinsichtlich der Instanziierung dieses KE und der semantischen Motivierung seiner KtE deutlich komplexer als etwa für die KtE des KE WEG der reflexiven Bewegungskonstruktion. Es können folgende vier Charakteristika, die in Abbildung 6.7 zusammengefasst sind, im Kontrast zur reflexiven Bewegungskonstruktion festgehalten werden.

a) Die Anzahl der Konstrukte mit einem relatierten lexikalischen Frame entspricht nicht automatisch der Anzahl der Konstrukte, in denen die KtE des KE 〈WEG〉 auch durch ein FE dieses lexikalischen Frames motiviert werden. Die Anzahl der Konstrukte mit einem relatierten lexikalischen Frame teilt sich somit noch einmal in diejenigen, in denen das KE 〈WEG〉 instanziiert ist, und in diejenigen, in denen es nicht instanziiert ist, auf.

b) Die Anzahl der Konstrukte mit einem relatierten lexikalischen Frame teilt sich ebenfalls in solche auf, in denen das $\mathrm{KE}\langle\mathrm{WEG}\rangle$ instanziiert ist und solche, in denen es nicht instanziiert ist. Konstrukte, in denen die KtE des KE 〈WEG〉 durch ein FE des Konstruktions-Frames (einfach oder doppelt) motiviert sind, stellen nicht, wie bei der reflexiven Bewegungskonstruktion, eine Teilmenge aller Konstrukte der Konstruktion dar, sondern nur eine Teilmenge der Kon- 
strukte, in denen das KE 〈WEG $\rangle$ überhaupt instanziiert ist. Ihre Verteilung über lexikalische Frames muss daher immer vor dem Hintergrund der Menge der Konstrukte, in denen das KE instanziiert ist (Tabellen 6.13 und 6.14) betrachtet werden.

c) Zusätzlich ergibt sich eine Menge an Konstrukten, in denen die KtE des KE 〈WEG〉 durch ein FE eines unrelatierten lexikalischen Frames motiviert wird und keine Beteiligung des Konstruktions-Frames stattfindet (vgl. Unterabschnitt 6.4.3). Dass es sich dabei nicht um Falschpositive handelt, kann daran erkannt werden, dass in anderen Konstrukten mit demselben unrelatierten lexikalischen Frame der Konstruktions-Frame an der Motivierung des KE $\langle\mathrm{WEG}\rangle$ - ebenso wie an anderen KE und dem KEE - beteiligt sein kann.

Insbesondere durch die beiden letzteren Punkte sind quantitative Angaben über die semantische Motivierung der KtE des $\mathrm{KE}\langle\mathrm{WEG}\rangle$ komplexer als diejenigen über die KtE des KE Weg der reflexiven Bewegungskonstruktion. Ich gehe deshalb in mehreren Schritten vor, um die noch ausstehenden quantitativen Verhältnisse der Instanziierung des $\mathrm{KE}$ 〈WEG〉 zu untersuchen. Bereits in den Tabellen 6.13 und 6.14 sind die Frequenzdaten zu den beiden oberen Ebenen in Abbildung 6.7 angegeben. $\mathrm{Zu}$ der für die reflexive Partikelverbkonstruktion noch in Unterabschnitt 7.5.2 festzustellenden hohen Produktivität passt die Beobachtung, dass die Mehrheit der insgesamt 850 Konstrukte dieser Konstruktion unrelatierte lexikalische Frames evoziert.

Zunächst jedoch zu den Konstrukten mit relatierten lexikalischen Frames. Deren Verteilung ist in Tabelle 6.16 dargestellt. Sie lässt sich weiter unterscheiden in eine fehlende Instanziierung des $\mathrm{KE}$ 〈WEG ) sowie in eine vorhandene Instanziierung des $\mathrm{KE}\langle\mathrm{WEG}\rangle$. Die Anzahl der Konstrukte mit instanziiertem KE $\langle\mathrm{WEG}\rangle$ entspricht zugleich derjenigen Anzahl an Konstrukten, in denen die KtE dieses und aller anderen KE einfach durch FE des betreffenden (relatierten) lexikalischen Frame motiviert sind, wie ich es in Unterabschnitt 6.2.1 für die reflexive Bewegungskonstruktion untersucht habe. Stellt man die gesamte Anzahl an Konstrukten für jeden lexikalischen Frame nun mit der jeweiligen Anzahl der Konstrukte ohne Instanziierung des KE $\langle\mathrm{WEG}\rangle$ gegenüber, stellt die Differenz aus beiden die Verteilung der Konstrukte mit instanziiertem KE $\langle\mathrm{WEG}\rangle$ dar, in denen zugleich die KtE aller anderen KE durch diese lexikalischen Frames motiviert werden.

Die Daten in Tabelle 6.16 zeigen, dass von insgesamt 304 Konstrukten mit relatierten lexikalischen Frames 168 ohne Instanziierung des KE 〈WEG〉 bleiben, während in 136 Konstrukten dieses KE instanziiert ist. Interessanterweise ist die Verteilung lexikalischer Frames in beiden Arten von Konstrukten dabei fast identisch, nur wenige Frames mit mittlerer und niedriger Frequenz tauschen untereinander die Plätze. 
Tab. 6.16: Konstrukte der reflexiven Partikelverbkonstruktion mit relatierten lexikalischen Frames

\begin{tabular}{lrrrrr}
\hline \multirow{2}{*}{ Lexikalischer Frame } & Gesamt & \multicolumn{2}{c}{$\langle$ KEG nicht instanziiert } & \multicolumn{2}{c}{$\langle$ WEG instanziiert } \\
& 118 & 83 & $49,40 \%$ & 35 & $25,74 \%$ \\
Body_movement & 56 & 26 & $15,48 \%$ & 30 & $22,06 \%$ \\
Self_motion & 53 & 25 & $14,88 \%$ & 28 & $20,59 \%$ \\
Cause_motion & 24 & 4 & $2,38 \%$ & 20 & $14,71 \%$ \\
Motion & 17 & 13 & $7,74 \%$ & 4 & $2,94 \%$ \\
Change_direction & 15 & 5 & $2,98 \%$ & 10 & $7,35 \%$ \\
Motion_directional & 11 & 5 & $2,81 \%$ & 6 & $4,40 \%$ \\
Placing & 8 & 6 & $3,57 \%$ & 2 & $1,47 \%$ \\
Bringing & 1 & 1 & $0,60 \%$ & & \\
Excreting & 1 & & & 1 & $0,74 \%$ \\
Operate_vehicle & 304 & 168 & $100,00 \%$ & 136 & $100,00 \%$ \\
\hline Gesamt & & & & &
\end{tabular}

Ein Blick auf die Konstrukte mit unrelatierten lexikalischen Frames zeigt, dass diese sich ebenso wie diejenigen mit relatierten Frames zunächst nach der Instanziierung des $\mathrm{KE}\langle\mathrm{WEG}\rangle$ aufspalten. Interessant sind hier wie bei der reflexiven Bewegungskonstruktion zunächst diejenigen unrelatierten lexikalischen Frames, die für die semantische Motivierung des KE 〈WEG $\rangle$ belegt sind, die also eine Verteilung derjenigen Konstrukte, in denen dieses KE instanziiert ist, aufzeigen. Dabei lassen sich, wie in Abbildung 6.7 angedeutet, analog zur reflexiven Bewegungskonstruktion (Unterabschnitte 6.2.2 und 6.2.3) eine einfache Motivierung der KtE des KE $\langle\mathrm{WEG}\rangle$ durch den Konstruktions-Frame sowie eine doppelte Motivierung der KtE durch lexikalischen Frame und Konstruktions-Frame unterscheiden. Diese beiden Fälle betrachte ich aus Gründen der Vergleichbarkeit mit der reflexiven Bewegungskonstruktion abschließend in diesem Unterabschnitt, bevor ich in mich in Unterabschnitt 6.4.3 einem für die reflexive Partikelverbkonstruktion besonderen Fall widme, in dem das KE 〈WEG einfach durch ein FE eines unprototpyischen lexikalischen Frames motiviert ist.

Von den insgesamt 249 Konstrukten mit unrelatierten lexikalischen Frames, in denen das KE 〈WEG) instanziiert ist (vgl. Tabelle 6.14), enthalten 49 eine einfache Motivierung des KtE durch ein FE des Konstruktions-Frames. Die Verteilung der lexikalischen Frames hierzu ist in Tabelle 6.17 dargestellt.

Vergleicht man die einfache Motivierung der KtE des KE $\langle\mathrm{WEG}\rangle$ mit derjenigen der KtE des KE WEG der reflexiven Bewegungskonstruktion (Unterabschnitt 6.2.2, Tabelle 6.3), ist zu erkennen, dass zahlreiche lexikalische Frames mit beiden Konstruktionen auftreten. Dazu zählen (in absteigender Rei- 
Tab. 6.17: Konstrukte der reflexiven Partikelverbkonstruktion mit einfacher Motivierung der KtE des KE $\langle\mathrm{WEG}\rangle$ durch den Konstruktions-Frame

\begin{tabular}{lrr}
\hline Lexikalischer Frame & Konstrukte & Anteil \\
\hline Daring & 11 & $22,45 \%$ \\
Hostile_encounter & 11 & $22,45 \%$ \\
Cause_to_experience & 6 & $12,24 \%$ \\
Manipulation & 4 & $8,16 \%$ \\
Shopping & 4 & $8,16 \%$ \\
Board_vehicle & 2 & $4,08 \%$ \\
Participation & 2 & $4,08 \%$ \\
Assistance & 1 & $2,04 \%$ \\
Attack & 1 & $2,04 \%$ \\
Grinding & 1 & $2,04 \%$ \\
Impact & 1 & $2,04 \%$ \\
Ingestion & 1 & $2,04 \%$ \\
Perception_experience & 1 & $2,04 \%$ \\
Processing_materials & 1 & $2,04 \%$ \\
Progression & 1 & $2,04 \%$ \\
Rescuing & 1 & $2,04 \%$ \\
\hline Gesamt & 49 & $100,00 \%$ \\
\hline
\end{tabular}

henfolge der Frequenz ihrer Konstrukte) die Frames Daring, Hostile_encounter, Cause_to_experience, Manipulation, Shopping, Impact und Ingestion. Da die Belege für diese lexikalischen Frames analog zu denen der reflexiven Bewegungskonstruktion sind, verzichte ich darauf, sie hier detailliert zu diskutieren. Stattdessen seien einige Belege für die weniger frequenten lexikalischen Frames Board_vehicle (108), Participation (109), Assistance (110), Attack (111), Grinding (112), Processing_materials (113) und Progression (114) zitiert.

(108) Sie [Board_vehicle Schiffte] sich an einem schönen Tag auch [zur Insel Brioni ${ }_{\text {GOAL }}$ ein, der berühmten Residenz erst Titos und später Tudjmans. (Die Zeit, 13.01.2000, Nr. 3)

(109) Man [participation klinkt] sich einfach [in die Konvention ${ }^{\mathrm{GOAL}}$ ] ein, ohne sich jemals „profiliert“, das heißt, von ihr unterschieden zu haben. (Schuh, Franz: Schreibkräfte, Köln: DuMont 2000, S. 174)

(110) Margarethe Sartoris, Tochter eines kleinen Angestellten, selbst Angestellte in einem Maschinenteilgroßhandel und Ehefrau eines kriegsinvaliden, früh schon ältlichen und gemütlichkeitsfixierten „Vereinsmeiers“, der sich [bis in die Leitungsebene einer Provinzsparkasse ${ }^{\mathrm{GOAL}}$ ] 
herauf[Assistance $\operatorname{dient}]$, ist klug, wach, couragiert und den schönen Künsten zugetan: (Die Zeit, 23.03.2000, Nr. 13)

(111) Der Präsident und seine Höflinge glaubten, die abtrünnige Republik ließe sich heim [ins russische Reich ${ }^{\text {GoAL }}$ [ [Attack bomben]. (Die Zeit, 05.01.2000, Nr. 2)

(112) Ein Motor dröhnt von der Straße herüber, und diese dröhnende Straße [Grinding fräst] sich [in mein Gedächtnis ${ }^{\mathrm{GOAL}}$ ] ein. (Riedel, Susanne: Eine Frau aus Amerika, Berlin: Berlin Verlag 2003, S. 95)

(113) Wir nahmen sie in die Mitte, von der Menschenmenge bedrängt, die die Runde auf den Hauptstraßen machte, Hand in Hand in Hand, zwei Stunden lang, während sich die Raketen über unseren Köpfen auffächerten, aus[Processing_materials färbten] [in die künstlichen Nächte ihrer Gestirne, Meteore, die unsichtbar am Horizont einschlugen GoAL]. (Schrott, Raoul: Tristan da Cunha oder die Hälfte der Erde; Hanser Verlag 2003, S. 260)

(114) Die zweite Schöpfung, die in der Enterprise nicht bloß aus Kontroll- und Waffensystemen, sondern auch aus Computermenschen mit Identitätsproblemen besteht und den Angriffen der intergalaktischen Maschinenmenschen namens „Borg“ im Dienste der „authentischen“ Menschen widerstehen muss, [progression entwickelt] sich konstant, aber höchst bedrohlich [in die Zukunft ${ }^{\text {GoAL}}$ ] hinein. (Die Zeit, 10.02.2000, Nr. 7)

Die Konstrukte, in denen KtE des instanziierten KE 〈WEG〉 doppelt motiviert werden, lassen sich wie bei der reflexiven Bewegungskonstruktion danach unterscheiden, welches FE des Konstruktions-Frames Motion in diese doppelte Motivierung eingeht. Wie bereits anhand der Daten in Tabelle $6.15 \mathrm{zu}$ erahnen ist, liegt eine deutliche Präferenz für das FE Motion.GoAL vor - nicht nur bezogen auf alle Konstrukte, an deren Motivierung Motion beteiligt ist, sondern auch speziell für die doppelte Motivierung der KtE des KE 〈WEG〉. In Tabelle 6.18 wird deutlich, dass das FE Motion.GoAL mit Abstand am frequentesten in doppelten Motivierungen auftritt, während die FE Motion.PATH (Tabelle 6.19) und Motion.SouRCE (Tabelle 6.20) in dieser Reihenfolge folgen. Das FE Motion.DiRECTION ist, anders als bei einfacher Motivierung, nicht unter den Konstrukten mit doppelter Motivierung der KtE des KE 〈WEG〉 belegt.

Auch hinsichtlich der doppelten Motivierung der KtE des KE 〈WEG〉 bietet sich ein Vergleich mit der reflexiven Bewegungskonstruktion an. Unter den lexikalischen Frames, die bei doppelter Motivierung mit dem FE Motion.GoAL belegt sind, sind Work, Cause_to_amalgamate, Desiring, Ingestion und Seeking für beide Konstruktionen belegt. Die im Folgenden zitierten Belege sind Beispiele für 
Tab. 6.18: Doppelte Motivierung der KtE des KE $\langle\mathrm{WEG}\rangle$ der reflexiven Partikelverbkonstruktion mit dem FE Motion.GoAL

\begin{tabular}{llrr}
\hline Lexikalischer Frame & FE & Konstrukte & Anteil \\
\hline Seeking & SOUGHT_ENTITY & 8 & $27,59 \%$ \\
Attaching & GOAL & 4 & $13,79 \%$ \\
Work & SALIENT_ENTITY & 4 & $13,79 \%$ \\
Cause_to_amalgamate & PART_2 & 3 & $10,34 \%$ \\
Cogitation & TOPIC & 2 & $6,90 \%$ \\
Desiring & EVENT & 2 & $6,90 \%$ \\
Experiencer_focus & CONTENT & 2 & $6,90 \%$ \\
Fire_burning & SUBREGION & 1 & $3,45 \%$ \\
Ingestion & INGESTIBLES & 1 & $3,45 \%$ \\
Manipulate_into_doing & RESULTING_ACTION & 1 & $3,45 \%$ \\
Questioning & TOPIC & 1 & $3,45 \%$ \\
\hline Gesamt & & 29 & $100,00 \%$ \\
\hline
\end{tabular}

die lexikalischen Frames Attaching (115), Cogitation (116), Experiencer_focus (117), Fire_burning (118), Manipulate_into_doing (119) und Questioning (120).

(115) a. Sie trug die Haare jetzt offen und [Attaching hängte] sich [GoAL bei ihm GoAL] ein. (Widmer, Urs: Das Buch des Vaters, Zürich: Diogenes 2004, S. 184)

b. Sie [Attaching henkelten] sich fest [Goal in den Armen ${ }^{\text {GoAl }}$ ] ein und verteilten sich auf die gesamte Straßenbreite. (Schulze, Ingo: Neue Leben, Berlin: Berlin Verlag 2005, S. 455)

(116) Lopomskis Methode, sich [Topıc in die Seelenzustände anderer GoAL] hineinzu[cogitation denken], bestand darin, den jeweiligen Gesichtsausdruck nachzuahmen und zu sehen, welche Gedanken und Empfindungen sich bei ihm einstellten. (Kopetzky, Steffen: Grand Tour, Frankfurt am Main: Eichborn 2002, S. 503)

(117) Kein Geringerer als Karl Marx stellte schon die Frage: „Ist die Presse frei, die sich [Content zum Gewerbe ${ }^{\text {GoAL}}$ ] herab[Experiencer_focus würdigt]?“ (Die Zeit, 17.02.2000, Nr. 8)

(118) Mit einer Nadel kratzt man hierauf die Konturen durchs Wachs und gießt Säure in diese fast unsichtbaren Furchen, die sich durchätzt und [SUBREGION im Kupfer GOAL] ein[Fire_burning brennt], die schwarze giftige Galle meiner Melancholie, mit der die wahren Umrisse der Welt einem so schmerzhaft anschaulich werden. (Schrott, Raoul: Tristan da Cunha oder die Hälfte der Erde; Hanser Verlag 2003, S. 133) 
Tab. 6.19: Doppelte Motivierung der KtE des KE $\langle\mathrm{WEG}\rangle$ der reflexiven Partikelverbkonstruktion mit dem FE Motion.PATH

\begin{tabular}{llrr}
\hline Lexikalischer Frame & FE & Konstrukte & Anteil \\
\hline Ingestion & INGESTIBLES & 1 & $25,00 \%$ \\
Manipulation & ENTITY & 1 & $25,00 \%$ \\
Processing_materials & MATERIAL & 1 & $25,00 \%$ \\
Work & SALIENT_ENTITY & 1 & $25,00 \%$ \\
\hline Gesamt & & 4 & $100,00 \%$ \\
\hline
\end{tabular}

Tab. 6.20: Doppelte Motivierung der KtE des KE $\langle$ WEG $\rangle$ der reflexiven Partikelverbkonstruktion mit dem FE Motion.SOURCE

\begin{tabular}{llrr}
\hline Lexikalischer Frame & FE & Konstrukte & Anteil \\
\hline Emptying & SOURCE & 1 & $50,00 \%$ \\
Filling & SSOURCE & 1 & $50,00 \%$ \\
\hline Gesamt & & 2 & $100,00 \%$ \\
\hline
\end{tabular}

(119) Die Tatsache aber, daß ich allein auf der Welt war, verlor ich auch in jener Zeit, als wir uns schon [Resulting_Action in die Spätvorstellungen GoaL] hinein[Manipulate_into_doing mogelten], nicht aus den Augen. (Stadler, Arnold: Sehnsucht, Köln: DuMont Literatur und Kunst Verlag 2002, S. 160)

(120) Katharina stellte ihr Glas ab und [Questioning fragte] sich [Topic zum Umkleideraum ${ }^{\text {GoAL }}$ ] durch. (Dölling, Beate: Hör auf zu trommeln, Herz, Weinheim: Beltz \& Gelberg 2003, S. 57)

Für die doppelte Motivierung mit dem FE Motion.PATH ist neben den bereits für die reflexive Bewegungskonstruktion belegten lexikalischen Frames Ingestion, Manipulation und Work noch Processing_materials belegt. Für diesen sei - gerade als Kontrast zu Beleg (113), in dem er mit einfacher Motivierung des KtE von $\langle$ WEG $\rangle$ durch den Konstruktions-Frame erscheint - mit (121) der einzige dafür vorhandene Beleg zitiert.

(121) Katharina fühlt die Tränen im Hals, die sie nicht hochkommen lassen will, sie kratzen und beißen und [Processing_materials ätzen] sich [MATERIAL die Speiseröhre ${ }^{\text {РАTH}] ~ h i n a b ~-~ d e n ~ O ̈ s o p h a g u s ~-, ~ w o ~ i s t ~ d e n n ~ i h r e ~ M u t t e r, ~}$ warum darf sie sie nicht einmal trösten? (Dölling, Beate: Hör auf zu trommeln, Herz, Weinheim: Beltz \& Gelberg 2003, S. 202) 
Zuletzt sind für die doppelte Motivierung mit dem FE Motion.SouRcE zwei lexikalische Frames belegt. Die folgenden Belege sind die einzigen belegten Beispiele für sie: Emptying (122) und Filling (123).

(122) Als ich die Augen schloß, war das Rot hinter meinen Lidern, und [Source aus ihm SouRCE] [Emptying schälten] sich diese Backsteinhäuser des Lagers heraus. (Kuckart, Judith: Lenas Liebe, Köln: DuMont Literatur und Kunst Verlag 2002, S. 55)

(123) Die leuchtendgelbe Schale einer Zitrone [Filling wickelt] sich spiralförmig [ ${ }_{\text {SOURCE }\rangle}$ von einer halben Frucht ${ }^{\text {SOURCE }}$ ] herunter und gibt das hellere Fleisch und die weißliche Innenhaut frei. (Noll, Ingrid: Ladylike, Zürich: Diogenes 2006, S. 289)

Als letzte Kategorie von Konstrukten der reflexiven Partikelverbkonstruktion hinsichtlich der Instanziierung des KE 〈WEG〉 fehlen noch diejenigen Konstrukte, die einen unrelatierten lexikalischen Frame evozieren und in denen das KE 〈WEG〉 nicht instanziiert ist. Tabelle 6.21 zeigt die Verteilung der lexikalischen Frames für diese Menge von Konstrukten.

$\mathrm{Zu}$ erkennen ist, dass viele der unrelatierten lexikalischen Frames, die bereits für Konstrukte mit instanziiertem KE 〈WEG〉 belegt sind, ebenfalls für Konstrukte belegt sind, in denen es nicht instanziiert ist. Ein abschließender Blick auf einige Beispiele kann sich deshalb auch hier auf lexikalische Frames beschränken, die allein innerhalb dieser Menge von Konstrukten belegt sind. Die folgenden Belege sind Beispiele für die Frames Chatting (124), Becoming_aware (125), Change_of_phase (126), Renting (127) und Temperature (128).

(124) a. Michaela [chatting redete] sich jedesmal heraus. (Schulze, Ingo: Neue Leben, Berlin: Berlin Verlag 2005, S. 577)

b. „Der Rektor [Chatting redet] sich raus“, sagt Berthold-Kuhles und sieht sich mittlerweile vor der Frage, „ob es für mich nicht günstiger ist, zum Sozialamt zu gehen“. (Die Zeit, 13.04.2000, Nr. 16)

c. Ein kritisierter Kritiker, der gegen eine an ihm geübte Kritik Erinnerungen an Goebbels wecken möchte, [chatting redet] sich bloß aus. (Schuh, Franz: Schreibkräfte, Köln: DuMont 2000, S. 112)

(125) Daß du dich so leicht durch[Becoming_aware findest], hätte ich nicht gedacht - toll. “(Kopetzky, Steffen: Grand Tour, Frankfurt am Main: Eichborn 2002, S. 194)

(126) Es sieht aus wie ein Körper, die Lunge des Magma sich gegen die Rippen pressend, ein und ausatmend, pochend wie Blut, anschwellend, sich aufstauend und das Land darüber anhebend, es auseinanderziehend, bis es 
Tab. 6.21: Konstrukte der reflexiven Partikelverbkonstruktion mit unrelatierten lexikalischen Frames ohne Instanziierung des KE $\langle$ WEG $\rangle$

\begin{tabular}{lrr}
\hline Lexikalischer Frame & Konstrukte & Anteil \\
\hline Cause_to_move_in_place & 250 & $84,18 \%$ \\
Daring & 13 & $4,38 \%$ \\
Work & 6 & $2,02 \%$ \\
Chatting & 5 & $1,68 \%$ \\
Impact & 3 & $1,01 \%$ \\
Cause_to_amalgamate & 2 & $0,67 \%$ \\
Hostile_encounter & 2 & $0,67 \%$ \\
Seeking & 2 & $0,67 \%$ \\
Becoming_aware & 1 & $0,34 \%$ \\
Cause_to_experience & 1 & $0,34 \%$ \\
Change_of_phase & 1 & $0,34 \%$ \\
Change_posture & 1 & $0,34 \%$ \\
Filling & 1 & $0,34 \%$ \\
Fire_burning & 1 & $0,34 \%$ \\
Ingestion & 1 & $0,34 \%$ \\
Make_noise & 1 & $0,34 \%$ \\
Manipulate_into_doing & 1 & $0,34 \%$ \\
Manipulation & 1 & $0,34 \%$ \\
Processing_materials & 1 & $0,34 \%$ \\
Questioning & 1 & $0,34 \%$ \\
Renting & 1 & $0,34 \%$ \\
Temperature & 1 & $0,34 \%$ \\
\hline Gesamt & 297 & $100,00 \%$ \\
\hline
\end{tabular}

dünn wird wie Haut, schließlich reißt und geschmolzenes Gestein sich nun durch[Change_of_phase schmilzt] und sich in die Grabenbrüche ergießt, von Neufundland über Brasilien bis nach Westafrika. (Schrott, Raoul: Tristan da Cunha oder die Hälfte der Erde; Hanser Verlag 2003, S. 522)

(127) Zwar standen pro Wohnung zwei Kinderzimmer bereit, was einer Aufforderung zur Fortpflanzung gleichkam, doch [Renting mieteten] sich auch dort wiederum nur alte Leute ein, die offenbar zu schwach und hinfällig waren, um gegen die reizlosen schuhkartonförmigen Siebziger-JahreRäume aufzubegehren. (Düffel, John von: Houwelandt, Köln: DuMont Literatur und Kunst Verlag 2004, S. 20)

(128) Ein Buch aus lauter tiefgefrorenen Kapiteln - Tödlicher Frost, man [Temperature friert] sich durch. (Die Zeit, 05.01.2000, Nr. 2) 
Unrelatierte lexikalische Frames treten in der reflexiven Partikelverbkonstruktion nicht nur dann auf, wenn ein KtE des KE 〈WEG〉 durch den Konstruktions-Frame einfach oder durch lexikalischen Frame und Konstruktions-Frame doppelt motiviert werden kann. Es kann ebenso der Fall sein, dass der unrelatierte lexikalische Frame ein KtE des KE 〈WEG〉 einfach motiviert, der Konstruktions-Frame also an der Konstitution eines Konstrukt-Frames - auch ,im Hintergrund' nicht beteiligt ist. Dieses Phänomen kann als ein Charakteristikum der reflexiven Partikelverbkonstruktion gelten.

\subsubsection{Einfache Motivierung durch unrelatierten lexikalischen Frame}

Für die reflexive Bewegungskonstruktion habe ich in den Unterabschnitten 6.2.1 und 6.3.1 festgestellt, dass die KtE aller ihrer Strukturelemente, der KE wie des KEE, einfach durch einen lexikalischen Frame motiviert sein können. Ausgangspunkt war dabei stets die Motivierung der KtE des KE WEG: Ist eines dieser KtE durch das FE eines lexikalischen Frames motiviert, ist es automatisch auch das KtE des KE BEWEGENDES sowie das KEE. ${ }^{92}$ Infrage kommen dafür allein relatierte lexikalische Frames, da sie aufgrund ihrer Frame-Nähe eine ,hintergründige“ Beziehung zum Konstruktions-Frame herstellen und die entsprechenden Instanzen eindeutig als Konstrukte der reflexiven Bewegungskonstruktion identifiziert werden können (dazu ausführlicher Unterabschnitte 8.2.2 und 8.4.2). Liegt hingegen ein unrelatierter lexikalischer Frame vor, lässt sich die Beteiligung des KonstruktionsFrames an der Motivierung der KtE des KE WEG ermitteln: Sie können einfach durch den Konstruktions-Frame (Unterabschnitt 6.2.2) oder doppelt motiviert (Unterabschnitt 6.2.3) sein.

Für die reflexive Partikelverbkonstruktion stellen sich diese Verhältnisse etwas anders dar. Zunächst gilt auch für sie, dass die Motivierung eines KtE des KE $\langle$ WEG〉 durch einen relatierten lexikalischen Frame darauf hinweist, dass auch das andere KtE sowie das KEE durch einen solchen Frame motiviert sind. Die KtE des KE 〈WEG $\rangle$ sind für diesen Fall als Ausgangspunkt der Analyse also weiterhin geeignet. Nun sind für die reflexive Partikelverbkonstruktion allerdings auch Frames belegt, bei der diese Feststellung an Grenzen stößt. Einige der unrelatierten

92 Es sei an dieser Stelle darauf hingewiesen, dass Einschätzungen wie diese selbstverständlich auf Annotationen aus vorherigen Analysen basieren und damit interpretationsabhängig sind. Zudem bewegen sich diese Interpretationen stets im Rahmen der Möglichkeiten der (für das Englische entwickelten) FrameNet-Datenbank, die gewisse Grenzen bei der Analyse setzt (dazu Unterabschnitt 2.1.3). Es ist also nicht gesagt, dass keine anderen als die im Folgenden diskutierten Ergebnisse erzielbar sind, wenn die Analyse nicht auf den gegebenen FrameNet-Daten basiert. 
lexikalischen Frames sind in der Lage, KtE des KE 〈WEG〉 bzw. Weg genauso wie relatierte lexikalische Frames einfach zu motivieren, also ohne einen Anteil des Konstruktions-Frames an der Motivierung dieses KtE. Dies betrifft für die reflexive Partikelverbkonstruktion drei lexikalische Frames, deren Verteilung in Tabelle 6.22 zusammengefasst ist.

Tab. 6.22: Einfache Motivierung der KtE des KE $\langle\mathrm{WEG}\rangle$ der reflexiven Partikelverbkonstruktion durch unrelatierte lexikalische Frames

\begin{tabular}{lrr}
\hline Lexikalischer Frame & Konstrukte & Anteil \\
\hline Cause_to_move_in_place & 161 & $97,60 \%$ \\
Burying & 2 & $1,20 \%$ \\
Filling & 2 & $1,20 \%$ \\
\hline Gesamt & 165 & $100,00 \%$ \\
\hline
\end{tabular}

Die Beobachtung, dass diese unrelatierten lexikalischen Frames in der Lage sind, KtE des KE 〈WEG $\rangle$ bzw. WEG einfach zu motivieren, wirft folgende Probleme auf.

a) Die KtE des KE 〈WEG〉 können nicht mehr uneingeschränkt als Indiz dienen, ob der Konstrukt-Frame eines Konstrukts der reflexiven Partikelverbkonstruktion bei einem unrelatierten lexikalischen Frame einen Anteil des Konstruktions-Frames enthält. ${ }^{93}$

b) Instanzen, die diese unrelatierten lexikalischen Frames evozieren, müssen weiterhin als Konstrukte der reflexiven Partikelverbkonstruktion in Erwägung gezogen werden, da sich der Anteil des Konstruktions-Frames auch an der Motivierung anderer Strukturelemente als der KtE des KE 〈WEG $\rangle$ zeigen kann.

c) Auch wenn durch die Analyse der Motivierung aller Strukturelemente festgestellt wird, dass der Konstruktions-Frame keinen Anteil an der Motivierung des Konstrukts hat, müssen Instanzen mit diesen unrelatierten lexikalischen Frames weiterhin als Konstrukte der reflexiven Partikelverbkonstruktion angesehen werden.

Zunächst zu Punkt a. Hierfür seien in (129) die Belege für den lexikalischen Frame Filling mit der reflexiven Partikelverbkonstruktion zitiert. In beiden sind die KtE des KE 〈WEG〉 einfach durch FE dieses Frames motiviert: Filling.GoAL und

93 Dieses Problem hat insbesondere Auswirkungen auf die Messung des Koerzionspotenzials für die reflexive Partikelverbkonstruktion, für die die KtE des KE 〈WEG〉 ebenfalls analog zu denjenigen des KE WEG der reflexiven Bewegungskonstruktion als Indiz dienen müssen. Ich komme darauf in den Unterabschnitten 7.4.1 und 7.4.3 zurück. 
Filling.〈SourCE〉. Eine Beteiligung der FE Motion.GoAL bzw. Motion.SourCE des Konstruktions-Frames erscheint unplausibel.

(129) a. In diesem Klang bist $\mathrm{Du}$ (für mich) ganz enthalten, alles andere ist nur ein Teil von dir, und wenn ich mich [GoAL in Deine Stimme] ein[Filling hülle], bist Du ganz bei mir. (Die Zeit, 30.03.2000, Nr. 14)

b. Hinter dem Dr.-Kurt-Fischer-Platz sah ich aus mehreren hundert Metern Entfernung die Straßenbahnen, die sich [〈SOURCE) vom Platz der Einheit] zurück[Filling stauten]. (Schulze, Ingo: Neue Leben, Berlin: Berlin Verlag 2005, S. 452)

Lässt sich in Konstrukten wie diesen dennoch eine Beteiligung des KonstruktionsFrames erkennen, wenn sie schon nicht in den KtE des KE 〈WEG〉 wirksam wird? Dies ist in der Tat möglich, wie der Einbezug anderer Strukturelemente nahelegt. Zum Vergleich sei deshalb mit (130) ein weiteres Konstrukt mit dem lexikalischen Frame Filling herangezogen, diesmal ohne Instanziierung des KE 〈WEG〉. Vor dem Hintergrund von Punkt b sei hier besondere Aufmerksamkeit der Motivierung des KEE geschenkt.

(130) Er [Filling schmierte] [Theme sich THEme] durch. (Kopetzky, Steffen: Grand Tour, Frankfurt am Main: Eichborn 2002, S. 456)

Obwohl das KEE bereits durch das FE Filling.THEME motiviert ist, erscheint es plausibel, dass auch das FE Motion.THEME des Konstruktions-Frames einen Anteil an der Motivierung des KEE hat. Deshalb ist in Beleg (130) eindeutig ein Konstrukt der reflexiven Partikelverbkonstruktion $\mathrm{zu}$ identifizieren, obwohl ein KtE des KE 〈WEG $\rangle$ hierfür nicht als Indiz herangezogen werden kann, da es in diesem Fall nicht instanziiert ist. Als Indiz kann hier stattdessen die Motivierung des KEE dienen. Ist an der Motivierung des KEE ein Anteil des Konstruktions-Frames zu erkennen, ist der lexikalische Frame weiterhin als unrelatiert und der Beleg gleichzeitig als ein Konstrukt der Konstruktion enthaltend zu identifizieren. Es liegt also weiterhin eine Koerzion des lexikalischen Frames durch den Konstruktions-Frame vor (dazu Unterabschnitt 7.4.3).

Was bedeutet das für die Konstrukte in (129)? Für die Motivierung des KEE ist festzustellen, dass dieses nicht wie in (130) doppelt motiviert ist, sondern einfach durch den lexikalischen Frame. Dasselbe gilt für die KtE des KE BEwEGENDES. Die vollständige Annotation ist in (131) dargestellt.

(131) a. In diesem Klang bist Du (für mich) ganz enthalten, alles andere ist nur ein Teil von dir, und wenn [AgEnT ich] [Theme mich] [GoAL in Deine 
Stimme] ein[Filling hülle], bist Du ganz bei mir. (Die Zeit, 30.03.2000, Nr. 14)

b. Hinter dem Dr.-Kurt-Fischer-Platz sah ich aus mehreren hundert Metern Entfernung die Straßenbahnen, [Agent die] [THEME sich] [(SourcE) vom Platz der Einheit] zurück[Filling stauten]. (Schulze, Ingo: Neue Leben, Berlin: Berlin Verlag 2005, S. 452)

Die Belege mit dem lexikalischen Frame Filling in (129) bzw. (131) sind vollständig durch einen unrelatierten lexikalischen Frame motiviert, ohne einen Anteil des Konstruktions-Frames Motion. Bedeutet dies, dass sie keine Konstrukte der reflexiven Partikelverbkonstruktion zeigen? Diese Frage leitet über zu Punkt c: Ich möchte dafür plädieren, dass diese Belege in der Tat Konstrukte der Konstruktion zeigen, auch wenn diese vollständig durch einen unrelatierten lexikalischen Frame motiviert sind. Der Grund dafür ist simpel. Sobald Belege mit demselben lexikalischen Frame zu finden sind, die - wie (130) - einen Anteil des KonstruktionsFrames erkennen lassen, gilt diese Feststellung für den gesamten lexikalischen Frame und damit auch für Belege, in denen dieser lexikalische Frame alle Strukturelemente des Konstrukts einfach motiviert. Alle Instanzen mit dem lexikalischen Frame Filling sind also als Konstrukte der reflexiven Partikelverbkonstruktion einzustufen, wenngleich lediglich in einem Teil davon eine tatsächliche Beteiligung des Konstruktions-Frames am Konstrukt-Frame zu erkennen ist.

Auf dieselbe Art und Weise lässt sich das Problem für Burying lösen. Auch hier kann eine plausible doppelte Motivierung des KEE festgestellt werden, wie die Belege in (132) zeigen.

(132) a. Es war wie das sichtbare Zeichen einer Gunst, dieses lichterfüllte Tuch, dessen Leichtigkeit und Geschmeidigkeit auf mich abstrahlte und mich spielend vereinte mit dem Meer, in dem ich schwamm, mit dem ich verschwamm, [GoAL in dessen Wellenberge und Täler] ich [THEME mich $^{\text {THEME }}$ ein[Burying grub], begünstigt und beglückt von seiner Güte. (Düffel, John von: Vom Wasser, München: dtv 2006, S. 251)

b. Doch England ist weit, und Einigkeit ist unter den Schiffbrüchigen auch damit nicht mehr zu erzielen; die seltenen klaren Tage verstreichen ungenützt, obwohl [THeme $_{\text {sich }}{ }^{\text {THEME }}$ ] der Hunger [GoAL in die Gesichter] ein[Burying gräbt]. (Schrott, Raoul: Tristan da Cunha oder die Hälfte der Erde; Hanser Verlag 2003, S. 280)

Ein besonderer Fall, der auch für die reflexive Bewegungskonstruktion gilt, stellt der Frame Cause_to_move_in_place dar (vgl. schon Unterabschnitt 6.2.2). Wie in Tabelle $6.22 \mathrm{zu}$ sehen, ist dieser für die reflexive Partikelverbkonstruktion äußerst 
frequent ohne Beteiligung des Konstruktions-Frames am Konstrukt-Frame belegt. Die drei Belege (133)-(135) für die LE abwenden, umdrehen und wegdrehen (Basisverben alle: turn.v) zeigen, dass alle Strukturelemente dieser Konstrukte durch Cause_to_move_in_place plausibel abgedeckt werden.

Als [Theme sich] [Agent der hagere Mann] [〈_irection) vom Fenster] ab[Cause_ to_move_in_place wand], erreichte der Minutenzeiger der großen Bahnhofsuhr das zweite Viertel. (Kopetzky, Steffen: Grand Tour, Frankfurt am Main: Eichborn 2002, S. 436)

(134) Dann fuhr er rechts ran, [Cause_to_move_in_place drehte] [Theme $\operatorname{sich}$ ] [(DiRection〉 zu ihr] um und fragte sie, ob sie nicht irgendwo was trinken gehen sollten. (Goosen, Frank: Liegen lernen, Frankfurt am Main: Eichborn AG 2000, S. 121)

(135) Er putzt die Linse noch einmal, [AGEnT er] [Cause_to_move_in_place dreht] [Theme sich] mit dem Belichtungsmesser [〈Direction) von der Sonne] weg, er kneift das linke Auge zu und schaut durchs Objektiv. (Beyer, Marcel: Spione, Köln: DuMont 2000, S. 167)

Wie für die Fälle von Filling und Burying muss nun auch für Cause_to_move_in_ place Evidenz gefunden werden, dass eine Beteiligung des Konstruktions-Frames an der Motivierung anderer Konstrukte möglich ist, sodass Konstrukte wie diejenigen in (133)-(135) weiterhin als solche der reflexiven Partikelverbkonstruktion gelten können. Anders als für die Fälle von Filling und Burying können dafür allerdings nicht Belege der reflexiven Partikelverbkonstruktion selbst herangezogen werden, da Cause_to_move_in_place über die in Tabelle 6.22 aufgelisteten Belege hinaus nicht für diese Konstruktion belegt ist. Hier kann nun die reflexive Bewegungskonstruktion als zusätzliche Evidenz dienen. Zwar ist Cause_to_move_in_place auch für sie in vollständiger Motivierung von Konstrukten belegt, allerdings existieren auch zwei Belege, in denen der KonstruktionsFrame einen Anteil besitzt. Diese seien in (136) zitiert.

(136) a. Sie [Cause_to_move_in_place wiegte] sich [gegen ihn GoAL]. (Hettche, Thomas: Der Fall Arbogast, Köln: DuMont Buchverlag, 2001, S. 14)

b. Als sie den Finger auf eine blaue Blüte schob, färbte sich ihr Fingernagel bläulich und grüne Streifen [Cause_to_move_in_place wellten] sich [über ihren Handrücken ${ }^{\mathrm{P}}{ }^{\mathrm{T} H}$ ]. (Pressler, Mirjam: Malka Mai, Weinheim Basel: Beltz \& Gelberg 2001, S. 5)

Dieser Fall zeigt, dass auch Konstrukte einer anderen verwandten Konstruktion als Evidenz dienen können, um zu ermitteln, ob Belege, die durch einen unrela- 
tierten lexikalischen Frame vollständig motiviert werden, Konstrukte der betreffenden Konstruktion zeigen. Für die reflexive Partikelverbkonstruktion kann somit davon ausgegangen werden, dass alle Instanzen mit dem lexikalischen Frame Cause_to_move_in_place auch dann als ihre Konstrukte zu werten sind, wenn an ihrer Motivierung kein Anteil des Konstruktions-Frames zu erkennen ist.

Klammert man besondere Fälle wie denjenigen des Frames Cause_to_move_ in_place aus, zeigt sich, dass insbesondere die semantische Motivierung des KEE ausschlaggebend für die Einstufung eines lexikalischen Frames als für die reflexive Partikelverbkonstruktion unrelatiert sein kann. Ich möchte deshalb analog zur reflexiven Bewegungskonstruktion (Abschnitt 6.3) abschließend auf jene semantische Motivierung des KEE für die reflexive Partikelverbkonstruktion eingehen. Wie bei der reflexiven Bewegungskonstruktion kann das KEE einfach durch einen lexikalischen Frame, einfach durch den Konstruktions-Frame oder doppelt motiviert sein. Voraussetzung für eine einfache Motivierung durch einen lexikalischen Frame ist auch hier, dass es sich um einen kausativen Frame handelt. Wie Tabelle 6.23 zeigt, sind drei Frames, durch deren FE das KEE einfach motiviert werden kann, belegt: Cause_motion, Placing und Bringing.

Tab. 6.23: Einfache Motivierung des KEE der reflexiven Partikelverbkonstruktion durch relatierte lexikalische Frames

\begin{tabular}{lrr}
\hline Lexikalischer Frame & Konstrukte & Anteil \\
\hline Cause_motion & 53 & $73,61 \%$ \\
Placing & 11 & $15,28 \%$ \\
Bringing & 8 & $11,11 \%$ \\
\hline Gesamt & 72 & $100,00 \%$ \\
\hline
\end{tabular}

Damit eine Instanz als Konstrukt der reflexiven Partikelverbkonstruktion gelten kann, in dem die Motivierung des KtE des KE 〈WEG〉 keinen Anteil des Konstruktions-Frames aufweist, muss das KEE entweder einfach durch den KonstruktionsFrame oder doppelt motiviert sein. Wie bei der reflexiven Bewegungskonstruktion sind die lexikalischen Frames, die mit dieser einfachen oder doppelten Motivierung des KEE auftreten, also besonders interessant. Tabelle 6.24 zeigt die lexikalischen Frames, die mit einer einfachen Motivierung des KEE durch das FE Motion.THEmE des Konstruktions-Frames auftreten. Tabelle 6.25 stellt die lexikalischen Frames und FE zusammen, die das KEE gemeinsam mit dem FE Motion.THEME doppelt motivieren.

Spätestens anhand der Verteilung lexikalischer Frames bei der Motivierung des KEE ist also zu erkennen, ob ein Frame zu für die reflexive Partikelverbkon- 
struktion belegten unrelatierten lexikalischen Frames zählt oder nicht. Schon auf der Basis des Vergleichs zwischen der Motivierung der KtE des KE 〈WEG $\rangle$ und derjenigen des KEE ist diese Entscheidung einfach zu treffen. Besondere Fälle wie diejenigen für Cause_to_move_in_place müssen indes eingehender betrachtet und bisweilen unter dem Vergleich mit Daten einer verwandten Konstruktion, hier der reflexiven Bewegungskonstruktion, entschieden werden.

Tab. 6.24: Einfache Motivierung des KEE der reflexiven Partikelverbkonstruktion durch den Konstruktions-Frame

\begin{tabular}{lrr}
\hline Lexikalischer Frame & Konstrukte & Anteil \\
\hline Daring & 24 & $23,53 \%$ \\
Hostile_encounter & 13 & $12,75 \%$ \\
Work & 11 & $10,78 \%$ \\
Seeking & 10 & $9,80 \%$ \\
Chatting & 5 & $4,90 \%$ \\
Impact & 4 & $3,92 \%$ \\
Ingestion & 4 & $3,92 \%$ \\
Shopping & 4 & $3,92 \%$ \\
Board_vehicle & 2 & $1,96 \%$ \\
Cogitation & 2 & $1,96 \%$ \\
Desiring & 2 & $1,96 \%$ \\
Experiencer_focus & 2 & $1,96 \%$ \\
Fire_burning & 2 & $1,96 \%$ \\
Manipulate_into_doing & 2 & $1,96 \%$ \\
Participation & 2 & $1,96 \%$ \\
Questioning & 2 & $1,96 \%$ \\
Assistance & 1 & $0,98 \%$ \\
Becoming_aware & 1 & $0,98 \%$ \\
Change_of_phase & 1 & $0,98 \%$ \\
Change_posture & 1 & $0,98 \%$ \\
Make_noise & 1 & $0,98 \%$ \\
Manipulation & 1 & $0,98 \%$ \\
Perception_experience & 1 & $0,98 \%$ \\
Processing_materials & 1 & $0,98 \%$ \\
Progression & 1 & $0,98 \%$ \\
Renting & 1 & $0,98 \%$ \\
Temperature & 1 & $0,98 \%$ \\
\hline Gesamt & 102 & $100,00 \%$ \\
\hline & &
\end{tabular}


Tab. 6.25: Doppelte Motivierung des KEE der reflexiven Partikelverbkonstruktion

\begin{tabular}{llrr}
\hline Lexikalischer Frame & FE & Konstrukte & Anteil \\
\hline Cause_to_experience & EXPERIENCER & 7 & $22,58 \%$ \\
Cause_to_amalgamate & PART_1 & 5 & $16,13 \%$ \\
Manipulation & ENTITY & 5 & $16,13 \%$ \\
Attaching & ITEM & 4 & $12,90 \%$ \\
Burying & THEME & 2 & $6,45 \%$ \\
Filling & THEME & 2 & $6,45 \%$ \\
Processing_materialss & MATERIAL & 2 & $6,45 \%$ \\
Attack & VICTIM & 1 & $3,23 \%$ \\
Emptying & THEME & 1 & $3,23 \%$ \\
Grinding & PATIENT & 1 & $3,23 \%$ \\
Rescuing & PATIENT & 1 & $3,23 \%$ \\
\hline Gesamt & & 31 & $100,00 \%$ \\
\hline & & \multicolumn{2}{c}{}
\end{tabular}

